\title{
A Mendelian randomization study of the role of lipoprotein subfractions in coronary artery disease
}

\author{
Qingyuan Zhao ${ }^{1}$, Jingshu Wang ${ }^{2}$, Zhen Miao $^{3}$, Nancy R Zhang ${ }^{4}$, Sean Hennessy ${ }^{3}$, Dylan S \\ Small $^{4}$, and Daniel J Rader ${ }^{3}$ \\ ${ }^{1}$ Statistical Laboratory, University of Cambridge \\ ${ }^{2}$ Department of Statistics, University of Chicago \\ ${ }^{3}$ Perelman School of Medicine, University of Pennsylvania \\ ${ }^{4}$ Department of Statistics, University of Pennsylvania
}

February 3, 2021

\begin{abstract}
Recent genetic data can offer important insights into the roles of lipoprotein subfractions and particle sizes in preventing coronary artery disease (CAD), as previous observational studies have often reported conflicting results. In this study, we first used the LD score regression to estimate the genetic correlation of 77 subfraction traits with traditional lipid profile and identified 27 traits that may involve distinct genetic mechanisms. We then used Mendelian randomization (MR) to estimate the causal effect of these traits on the risk of CAD. In univariable MR, the concentration and content of medium high-density lipoprotein (HDL) particles showed a protective effect against coronary artery disease. The effect was not attenuated in multivariable MR that adjusted for traditional lipid profile. The multivariable MR analyses also found that small HDL particles and smaller mean HDL particle diameter may have a protective effect. We identified four genetic markers for HDL particle size and CAD.
\end{abstract}

\section{Introduction}

Lipoprotein subfractions have been increasingly studied in epidemiological research and used in clinical practice to predict the risk of cardiovascular diseases (CVD) 1-3. . Several studies have identified potentially novel subfraction predictors for CVD [2, 4, 8, and demonstrated that the addition of subfraction measurements can significantly improve the risk prediction for CVD [1, 9-11]. However, these observational studies often provide conflicting evidence on the precise roles of the lipoprotein subfractions. For example, while some studies suggested that small, dense low-density lipoprotein (LDL) particles may be more atherogenic [4, 12], others found that larger LDL size is associated with higher CVD risk [13, 14]. Some recent observational studies found that the inverse association of CVD outcomes with smaller high-density lipoprotein (HDL) particles is stronger than the association with larger HDL particles [6, 11, 15, 16, but other studies reached the opposite conclusion in different cohorts 17, 18. Currently, the utility of lipoprotein subfractions or particle sizes in routine clinical practice remains controversial 14, 19-21, as there is still a great uncertainty about their causal roles in CVD, largely due to a lack of intervention data [21].

Mendelian randomization (MR) is an useful causal inference method that avoids many common pitfalls of observational cohort studies 22. By using genetic variation as instrumental variables, MR asks if the genetic predisposition to a higher level of the exposure (in this case, lipoprotein subfractions) is associated with higher occurrences of the disease outcome [23]. A positive association suggests a causally protective effect of the exposure if the genetic variants satisfy the instrumental variable assumptions [23, 24]. Since Mendelian randomization can provide unbiased causal estimate even when there are unmeasured confounders, it is generally considered more credible than other non-randomized designs and is quickly gaining popularity in epidemiological research [25, 26]. MR has been used to estimate the effect of several metabolites on CVD, but most prior studies are limited to just one or a few risk exposures at a time [27, 28].

In this study, we will use recent genetic data to investigate the roles of lipid and lipoprotein traits in the occurrence of coronary artery disease (CAD) and myocardial infarction (MI). In particular, we are interested in 
Table 1: Information about the GWAS summary datasets used in this article. The columns are the phenotypes reported by the GWAS studies, the consortium or name of the first author of the publication, PubMed ID, population, sample size, other GWAS datasets with other lapping sample, and URLs we used to download the datasets.

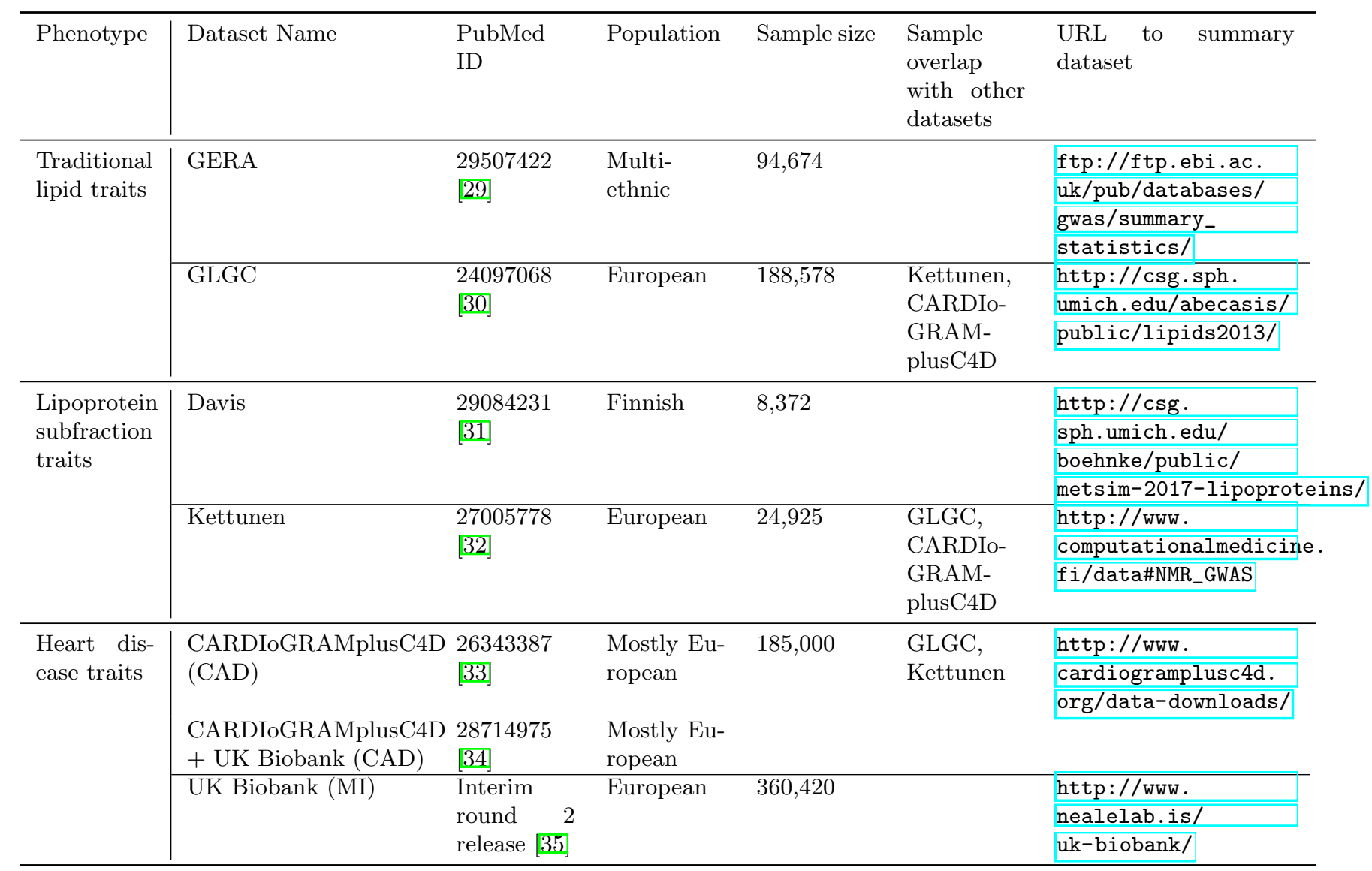

discovering lipoprotein subfractions that may be causal risk factors for CAD and MI in addition to the traditional lipid profile (LDL cholesterol, HDL cholesterol, and triglycerides levels). To this end, we will first estimate the genetic correlation of the lipoprotein subfractions and particle sizes with the tradition risk factors and remove the traits that have a high genetic correlation. We will then use MR to estimate the causal effects of the selected lipoprotein subfractions and particle sizes on CAD and MI. Finally, we will explore potential genetic markers for the identified lipoprotein and subfraction traits.

\section{Materials and Methods}

\section{GWAS summary datasets and lipoprotein particle measurements}

Table 1 describes all GWAS summary datasets used in this study, including two GWAS of the traditional lipid risk factors [29, 30, two recent GWAS of the human lipidome [31, 32], and three GWAS of CAD or MI [33-35]. In the two GWAS of the lipidome [31, 32, high-throughput nuclear magnetic resonance (NMR) spectroscopy was used to measure the circulating lipid and lipoprotein traits [36]. We investigated the 82 lipid and lipoprotein traits measured in these studies that are related to very-low-density lipoprotein (VLDL), LDL, intermediatedensity lipoprotein (IDL) and HDL subfractions and particle sizes. All the subfraction traits are named with three components that are separated by hyphens: the first component indicates the size (XS, S, M, L, XL, XXL); the second component indicates the fraction according to the lipoprotein density (VLDL, LDL, IDL, HDL); the third component indicates the measurement ( $\mathrm{C}$ for total cholesterol, $\mathrm{CE}$ for cholesterol esters, FC for free cholesterol, $\mathrm{L}$ for total lipids, P for particle concentration, PL for phospholipids, TG for triglycerides). For example, M-HDL-P refers to the concentration of medium HDL particles.

Aside from the concentration and content of lipoprotein subfractions, the two lipidome GWAS also measured 
the traditional lipid traits (TG, LDL-C, HDL-C), the average diameter of the fractions (VLDL-D, LDL-D, HDL-D) and the concentration of apolipoprotein A1 (ApoA1) and apolipoprotein B (ApoB). A full list of the lipoprotein measurements investigated in this article can be found in Appendix 1.

\section{Genetic correlation and phenotypic screening}

Genetic correlation is a measure of association between the genetic determinants of two phenotypes. It is generally different from epidemiological correlation that is estimated from cross-sectional data. In this study, we applied the LD-score regression [37] to the lipidome GWAS [31, 32] to estimate the genetic correlations between the lipoprotein subfractions, particle sizes, and traditional risk factors. We then removed lipoprotein subfractions and particle sizes that are strongly correlated with the traditional risk factors, defined as an estimated genetic correlation i 0.8 with TG, LDL-C, HDL-C, ApoB, or ApoA1 in the GWAS published by Davis et al. 31]. Because these traits are largely co-determined with the traditional risk factors, they do not represent independent biological mechanisms and may lead to multicollinearity issues in multivariate MR analyses. Finally, we obtained an independent estimate of the genetic correlations between the selected traits by applying the LD score regression to the GWAS published by Kettunen et al. [32]. We used Bonferroni's procedure to correct for multiple testing (familywise error rate at 0.05).

\section{Three-sample Mendelian randomization design}

For Mendelian randomization, we employed a three-sample design [38] in which one GWAS was used to select independent genetic instruments that are associated with one or several lipoprotein measures. The other two GWAS were then used to obtain summary associations of the selected SNPs with the exposure and the outcome, as in a typical two-sample MR design [39, 40]. More specifically, the selection GWAS was used to create a set of SNPs that are in linkage equilibrium with each other in a reference panel (distance $>10$ megabase pairs, $r^{2}<0.001$ ). This was done by ordering the SNPs by the p-values of their association with the trait(s) under investigation and then selecting them greedily using the linkage-disequilibrium (LD) clumping function in the PLINK software package [41. To avoid winner's curse, we require the other two GWAS to have no overlapping sample with the selection GWAS.

As the GWAS published by Davis et al. 31] has a smaller sample size, we used it to select the genetic instruments so the larger dataset can be used for statistical estimation. In univariable MR, associations of the selected SNPs with the exposure trait (a lipoprotein subfraction or a particle size trait) were obtained from the GWAS published by Kettunen et al. 32] and the associations with MI were obtained using summary data from an interim release of UK BioBank [35]. To maximize the statistical power, we used the so-called "genome-wide MR" design and do not truncate the list of SNPs according to their p-values. More details about this design can be found in a previous methodological article [38.

To control for potential pleiotropic effects via the traditional risk factors, we performed two multivariable MR analyses for each lipoprotein subfraction or particle size under investigation. The first multivariable MR analysis considers four exposures: TG, LDL-C, HDL-C, and the lipoprotein measurement under investigation. The second multivariable MR analysis replaces LDL-C and HDL-C with ApoB and ApoA1, in accordance with some recent studies [42]. SNPs were ranked by their minimum p-values with the four exposures and are selected as instruments only if they were associated with at least one of the four exposures (p-value $\leq 10^{-4}$ ). Both multivariable MR analyses used the Davis [31] and GERA [29] datasets for instrument selection, the Kettunen [32] and GLGC [30] datasets for the associations of the instruments with the exposures, and the CARDIoGRAMplusC4D + UK Biobank [34] dataset for the associations with CAD.

\section{Statistical estimation}

For univariable MR, we used the robust adjusted profile score (RAPS) because it is more efficient and robust than many conventional methods [38, 43]. RAPS can consistently estimate the causal effect even when some of the genetic variants violate instrumental variables assumptions. For multivariable MR, we used an extension to RAPS called GRAPPLE to obtain the causal effect estimates of multiple exposures [44]. GRAPPLE also allows the exposure GWAS to have overlapping sample with the outcome GWAS, while the original RAPS does not. We assessed the strength of the instruments using the modified Cochran's Q statistic [45]. Because many lipoprotein subfraction traits were analyzed simultaneously, we used the Benjamini-Hochberg procedure to correct for multiple testing [46] and the false discovery rate was set to be 0.05. More detail about the statistical methods can be found in Appendix 3. 


\section{Genetic markers}

To obtain genetic markers, we selected SNPs that are associated with the lipoprotein measurements identified in the MR (p-value $\leq 5 \times 10^{-8}$ ) and CAD (p-value $\left.\leq 0.05\right)$ but are not associated with LDL-C or ApoB (p-value $\geq 10^{-3}$ ). To maximize the power of this exploratory analysis, we meta-analyzed the results of the two lipidome GWAS [31, 32] by inverse-variance weighting. For the associations with LDL-C and CAD, we used the GWAS summary data reported by the GLGC [30] and CARDIoGRAMplusC4D [34] consortia. We used LD clumping to obtain independent markers [41] and then validate the markers using tissue-specific gene expression data from the GTEx project.

\section{Sensitivity analysis and replicability}

Because we had multiple GWAS summary datasets for the lipoprotein subfractions and CAD/MI (Table 1), we swapped the roles of the GWAS datasets in the three-sample MR design whenever permitted by the statistical methods to obtain multiple statistical estimates. These estimates are not completely independent of the primary results, but they can nonetheless be used to assess replicability. As a sensitivity analysis, We further analyzed univariable MR using inverse-variance weighting (IVW) [47] and weighted median [48] and compared with the primary results obtained by RAPS. We also assessed the assumptions made by RAPS using some diagnostic plots suggested in previous methodological articles [38].

\section{Results}

\section{Genetic correlations and phenotypic screening}

We obtained the genetic correlations of the lipoprotein subfractions and particle sizes with the traditional lipid risk factors: TG, LDL-C, HDL-C, ApoB, and ApoA1 (Table 1). We found that almost all VLDL subfractions traits (besides those related to very small VLDL subfraction) and the mean VLDL particle diameter have an estimated genetic correlation with TG very close to 1 . Most traits related to the large and very large HDL subfractions also have a high genetic correlation with HDL-C and ApoA1.

After removing traits that are strongly correlated with the traditional risk factors, we obtained 27 traits that may involve independent genetic mechanisms. Figure 1 shows the genetic correlation matrix for these traits and the traditional lipid factors. The selected traits can be divided into two groups based on whether they are related to VLDL/LDL/IDL particles or HDL particles. Within each group, most traits were strongly correlated with the others. In the first group, most traits had a positive genetic correlation with LDL-C and ApoB, while in the second group, most traits had a positive genetic correlation with HDL-C and ApoA1. Exceptions include LDL-D, which had a negative but statistically non-significant genetic correlation with LDL-C and ApoB, and S-HDL-P and S-HDL-L, which showed no or weak genetic correlation with HDL-C and ApoA1.

\section{Mendelian randomization}

Figure 2 shows the estimated causal effect of the selected lipoprotein measurements on MI or CAD that are statistically significant (false discovery rate $=0.05$ ). The unfiltered results can be found in Appendix 3 , which also contains results of the sensitivity and replicability analyses.

The concentration and lipid content of VLDL, LDL, and IDL subfractions showed harmful and nearly uniform effects on MI in univariable MR. However, after adjusting for the traditional lipid risk factors, the effects of these ApoB-related subfractions become close to zero (besides IDL-FC in one multivariable analysis). The mean diameter of LDL particles (LDL-D) showed a harmful effect on MI in univariable MR, though the effect was smaller than those of the LDL subfractions in univariable MR. The estimated effect of LDL-D was attenuated in the multivariable MR analyses.

The concentration and content of medium HDL particles showed protective effects in univariable and multivariable MR analyses. In particular, adjusting for the traditional lipid risk factors did not attenuate the effect of traits related to medium HDL. The concentration of and total lipid in small HDL particles showed protective effects in multivariable MR analyses, though the effect sizes were smaller than those of the medium HDL traits. The mean diameter of HDL particles (HDL-D) had almost no effect on MI in the univariable MR analysis, but after adjusting for the traditional lipid risk factors, it showed a harmful effect.

Table 2 reports the estimated effects of M-HDL-P, S-HDL-P, HDL-D, and traditional lipid traits (TG, LDL-C, HDL-C, ApoB, ApoA1) in the multivariable MR analyses. To better understand the role of HDL subfractions and 
bioRxiv preprint doi: https://doi.org/10.1101/691089; this version posted February 6, 2021. The copyright holder for this preprint (which was not certified by peer review) is the author/funder, who has granted bioRxiv a license to display the preprint in perpetuity. It is made available under aCC-BY-NC-ND 4.0 International license.

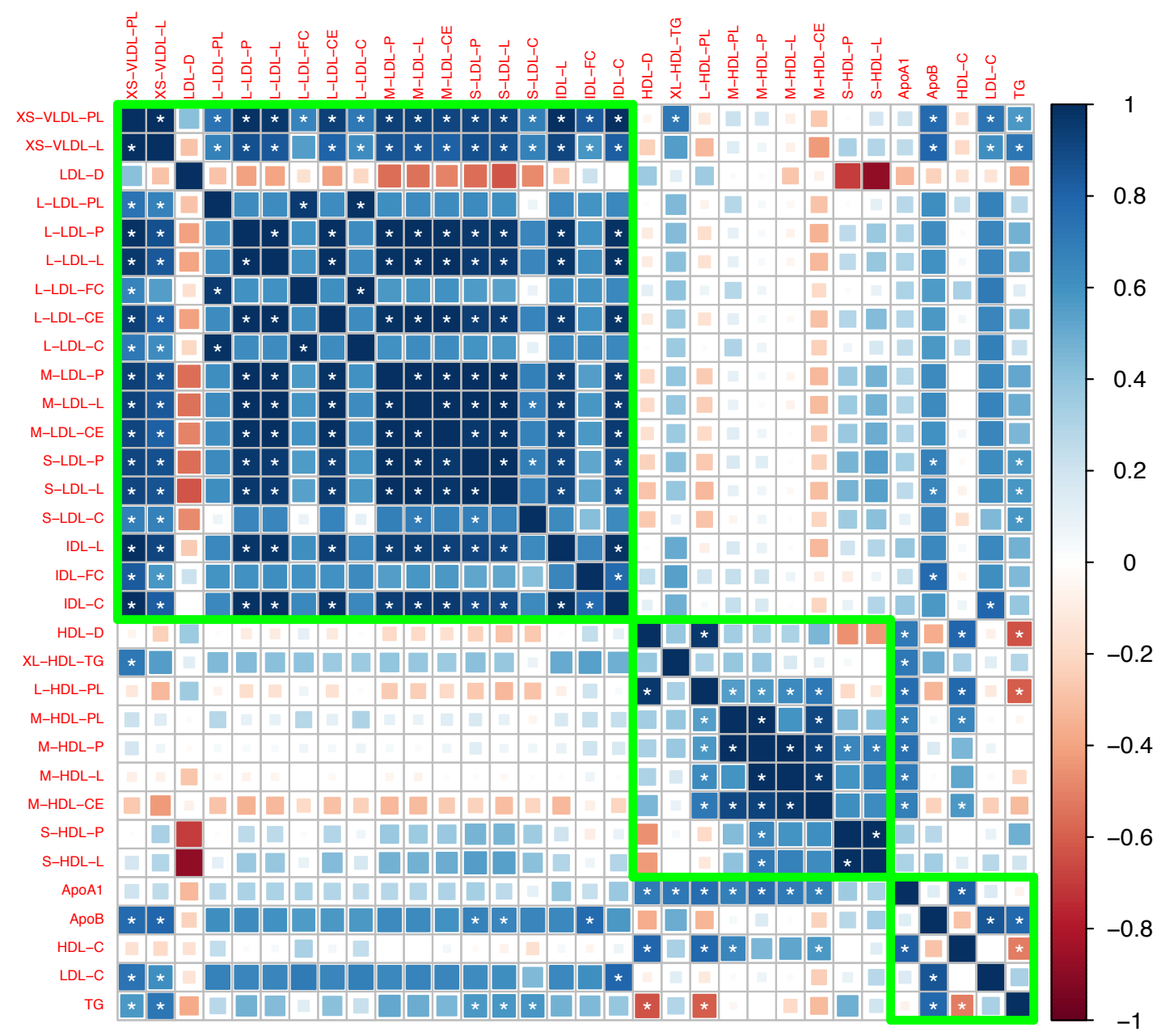

Figure 1: Genetic correlation matrix of the 27 lipoprotein subfraction traits selected in phenotypic screening and 5 traditional lipid traits. White asterisk indicates the correlation is statistically significant after Bonferroni correction for multiple comparisons at level 0.05 .

particle sizes, we also included in the table the results of the multivariate MR analyses for the traditional lipid risk factors only. Those baseline analyses suggested that HDL-C/ApoA1 had a weak, non-significant protective effect on CAD, which is consistent with prior studies [44, 49]. Adding S-HDL-P to the MR analysis did not substantially alter the estimated effects of the traditional lipid traits. However, when M-HDL-P or HDL-D was included in the model, the estimated effects of M-HDL-P and HDL-D changed substantially. In particular, when M-HDL-P was included in the multivariable MR analyses, HDL-C/ApoA1 showed a harmful effect on CAD. When HDL-D was included, HDL-C/ApoA1 showed a protective effect.

\section{Genetic markers associated with HDL and CAD}

We identified four genetic variants that are associated with S-HDL-P, M-HDL-P, or HDL-D, not associated with LDL-C or ApoB, and associated with CAD: rs838880 (SCARB1), rs737337 (DOCK6), rs2943641 (IRS1), and rs6065904 (PLTP) (Figure 3). These SNP-cis gene pairs are also supported by examining expression quantitative trait loci (eQTL) in the tissue-specific GTEx data Appendix 4). The first three variants were not associated with SHDL-P. However, they had uniformly positive associations with M-HDL-P, L-HDL-P, XL-HDL-P, HDL-D, ApoA1, and HDL-C, and a negative association with CAD. The last variant rs6065904 had positive associations with S- 

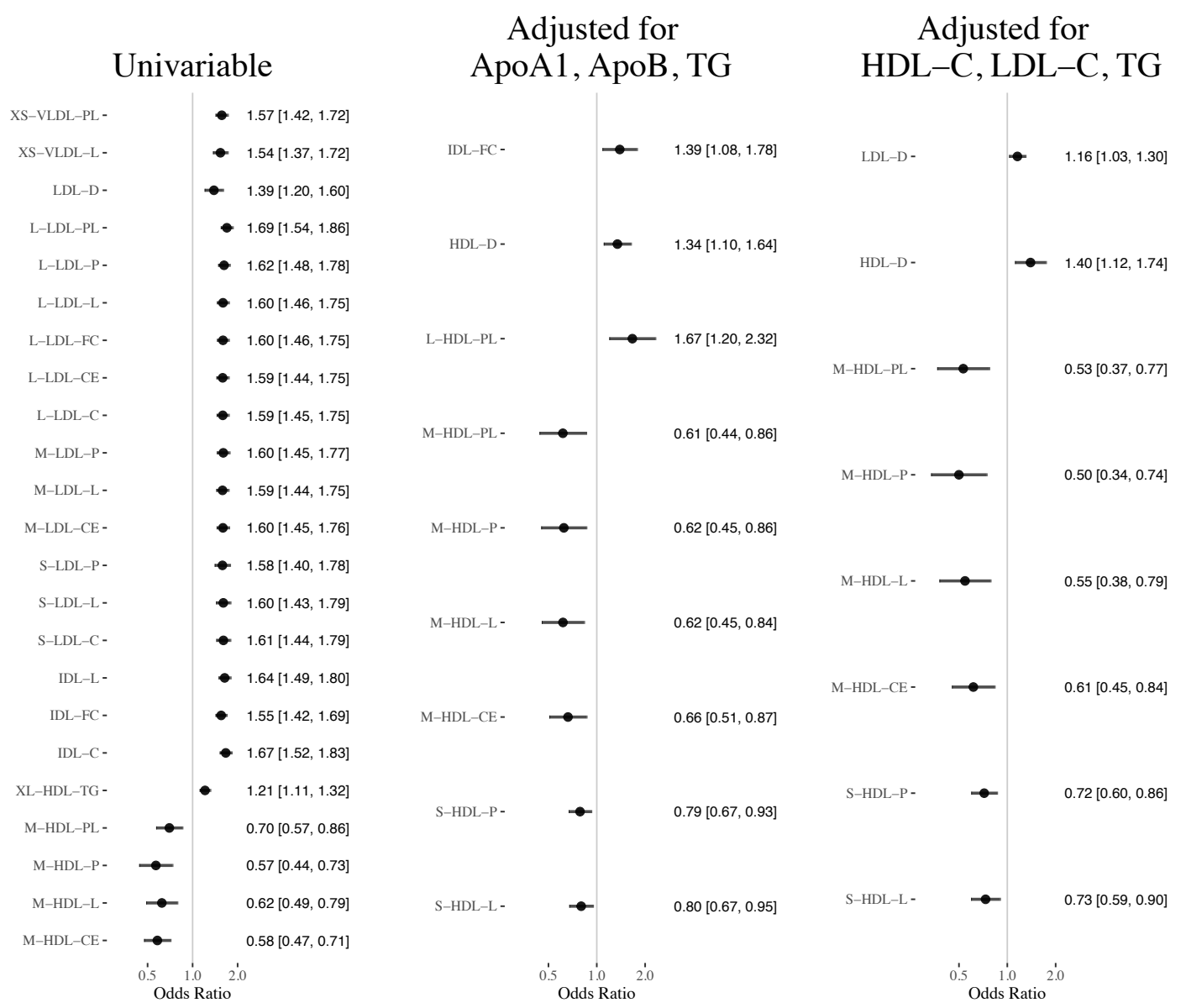

Figure 2: Results of the Mendelian randomization analyses (false discover rate $=0.05$ ): Estimated odds ratio $[95 \%$ confidence interval] per standard deviation increase of the selected lipoprotein measurements on MI or CAD.

HDL-P and M-HDL-P, negative associations with L-HDL-P, XL-HDL-P, HDL-D, negative but smaller associations with ApoA1 and HDL-C, and a negative association with CAD.

\section{Sensitivity and replicability analysis}

We also investigated the effects of lipoprotein subfractions and particle sizes on MI/CAD using multiple GWAS datasets, MR designs and statistical methods. The results are provided in Appendix 3 and are generally in agreement with the primary results reported above. The diagnostic plots for S-HDL-P and M-HDL-P did not suggest evidence of violations of the instrument strength independent of direct effect (InSIDE) assumption [50] made by RAPS and GRAPPLE Appendix 4.

\section{Discussion}

By using recent genetic data and Mendelian randomization, this study examines whether some lipoprotein subfractions and particle sizes, beyond the traditional lipid risk factors, may play a role in coronary artery disease. We find that VLDL subfractions have extremely high genetic correlations with blood triglyceride level and thus offer little extra value. We find some weak evidence that larger LDL particle size may have a small harmful effect on myocardial infarction and coronary artery disease.

Our main finding is that the size of HDL particles may play an important and previously undiscovered role. Although the concentration and lipid content of small and medium HDL particles appear to be positively correlated with HDL cholesterol and ApoA1, their genetic correlations are much smaller than 1, indicating the existence of independent biological pathways. Moreover, the Mendelian randomization analyses suggested that the small and 
Table 2: Results of some multivariable Mendelian randomization analyses. Each row in the table corresponds to a multivariable MR analysis with traditional lipid profile and the specified lipoprotein subfraction or particle size trait. Reported numbers are the point estimates (standard error) of the exposure effect.

\begin{tabular}{l|llll}
\hline Trait & Effect of TG & Effect of LDL-C & Effect of HDL-C & Effect of Subfraction/Particle size \\
\hline None & $0.192(0.051)$ & $0.383(0.027)$ & $-0.053(0.041)$ & \\
M-HDL-P & $0.371(0.075)$ & $0.386(0.033)$ & $0.302(0.112)$ & $-0.694(0.204)$ \\
S-HDL-P & $0.225(0.056)$ & $0.453(0.035)$ & $-0.112(0.046)$ & $-0.331(0.095)$ \\
HDL-D & $0.111(0.055)$ & $0.421(0.033)$ & $-0.442(0.124)$ & $0.333(0.114)$ \\
\hline & Effect of TG & Effect of ApoB & Effect of ApoA1 & Effect of Subfraction/Particle size \\
\hline None & $0.047(0.049)$ & $0.488(0.055)$ & $-0.095(0.059)$ & \\
M-HDL-P & $-0.004(0.090)$ & $0.501(0.097)$ & $0.129(0.097)$ & $-0.472(0.166)$ \\
S-HDL-P & $0.071(0.050)$ & $0.531(0.059)$ & $-0.132(-.059)$ & $-0.240(0.083)$ \\
HDL-D & $0.056(0.049)$ & $0.613(0.073)$ & $-0.457(0.138)$ & $0.296(0.100)$ \\
\hline
\end{tabular}

medium HDL particles may have protective effects on CAD. We also find that larger HDL mean particle diameter may have a harmful effect on CAD. Finally, we identified four potential genetic markers for HDL particle size that are independent of LDL cholesterol and ApoB.

There has been a heated debate on the role of HDL particles in CAD in recent years following the failure of several trials for CETP inhibitors [51/53] and recombinant ApoA1 [54] targeting HDL cholesterol. Observational epidemiology studies have long demonstrated strong inverse association between HDL cholesterol and the risk of CAD or MI [55-57, but conflicting evidence has been found in MR studies. In an influential study, Voight and collaborators found that the genetic variants associated with HDL cholesterol had varied associations with CAD and that almost all variants suggesting a protective effect of HDL cholesterol were also associated with LDL cholesterol or triglycerides [58]. Other MR studies also found that the effect of HDL cholesterol on CAD is heterogeneous [38] or attenuated after adjusting for LDL cholesterol and triglycerides [59, 60].

Notice that the harmful effect of larger HDL particle diameter found in this study relies on including HDL-C or ApoA1 in the multivariable MR analysis. Thus, the role of HDL particles in preventing CAD may be more complicated than, for example, that of LDL cholesterol or ApoB. It is possible that HDL cholesterol, HDL subfractions, and HDL particle size are all phenotypic markers for some underlying causal mechanism. A related theory is the HDL function hypothesis [61. Cholesterol efflux capacity, a measure of HDL function, has been documented as superior to HDL-C in predicting CVD risk [62, 63. Recent epidemiologic studies found that HDL particle size is positively associated with cholesterol efflux capacity in post-menopausal women [64 and in an asymptomatic older cohort 65. However, mechanistic efflux studies showed that small HDL particles actually mediate more cholesterol efflux [66, 67]. A likely explanation of this seeming contradiction is that a high concentration of small HDL particles in the serum may mark a block in maturation of small HDL particles [65]. This can also partly explain our finding that small HDL traits have a smaller effect than medium HDL traits, as increased medium HDL might indicate successful maturation of small HDL particles.

Among the reported genetic markers, SCARB1 and PLTP have established relations to HDL metabolism and CAD. SCARB1 encodes a plasma membrane receptor for HDL and is involved in hepatic uptake of cholesterol from peripheral tissues. Recently, a rare mutation (P376L) of SCARB1 was reported to raise HDL-C level and increase CAD risk 68, 69. This is opposite direction to the conventional belief that HDL-C is protective and could be explained by HDL dysfunction. PLTP encodes the phospholipid transfer protein and mediates the transfer of phospholipid and cholesterol from LDL and VLDL to HDL. As a result, PLTP plays a complex but pivotal role in HDL particle size and composition. Several studies have suggested that high PLTP activity is a risk factor for CAD [70-72].

Our study should be viewed in the context of its limitations, in particular, the inherent limitations of the summary-data Mendelian randomization design. Any causal inference from non-experimental data makes unverifiable assumptions, so does our study. Conventional MR studies assume that the genetic variants are valid instrumental variables. The statistical methods used by us make less stringent assumptions about the instrumental variables, but those assumptions could still be violated even though our model diagnosis does not suggest evidence against the InSIDE assumption. Our study did not adjust for other risk factors for CAD such as body mass index, blood pressure, and smoking. All the GWAS datasets used in this study are from the European population, so the same conclusions might not generalize to other populations. Furthermore, our study used GWAS datasets from 


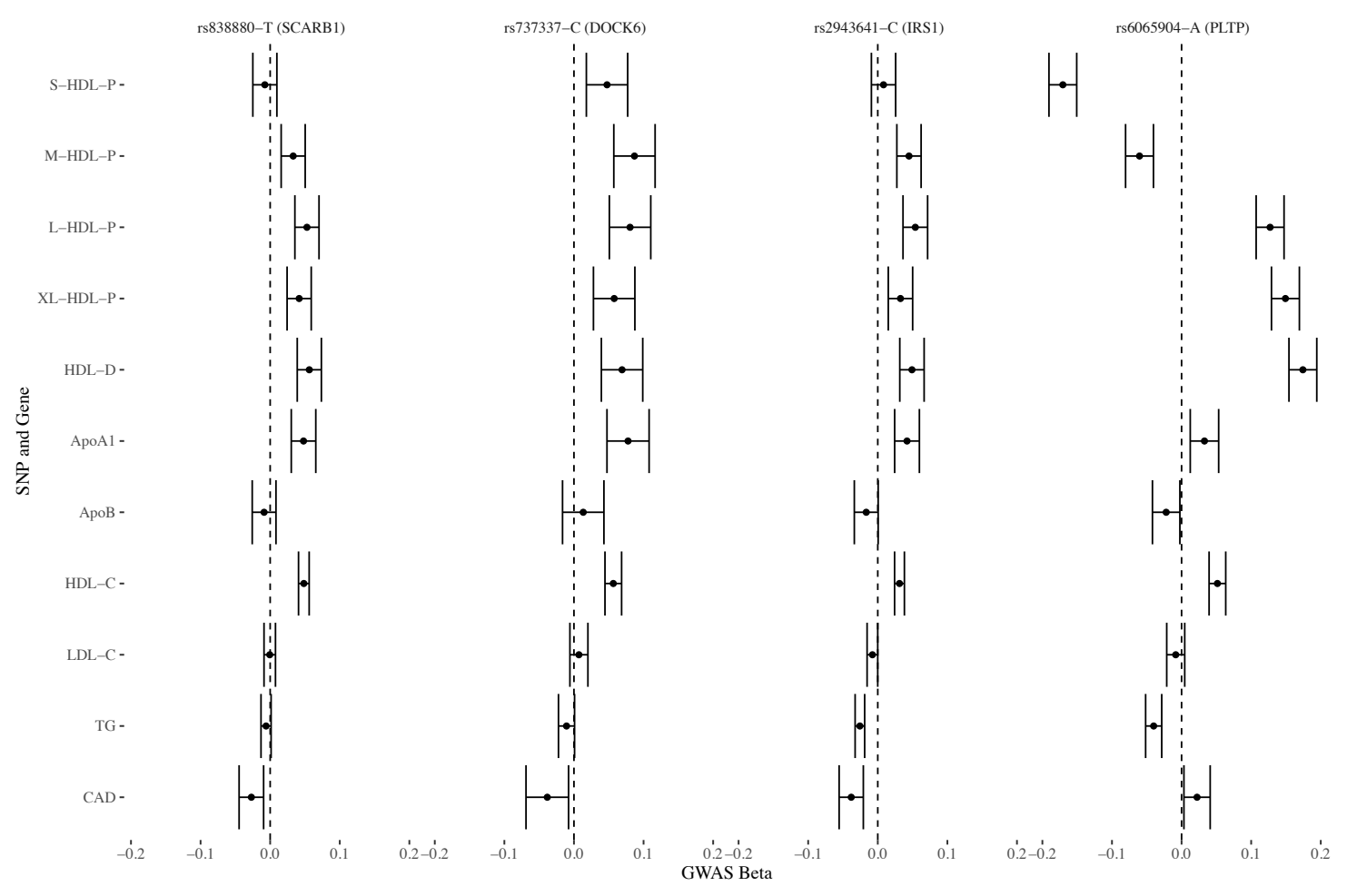

Figure 3: Genetic markers for HDL size (with risk alleles) and their associations with various lipid traits.

heterogeneous subpopulations, which may also introduce bias [73. We also did not use more than one subfraction traits as exposures in multivariable MR because of their high genetic correlations. Alternative statistical methods could be used to select the best causal risk factor from high-throughput experiments [74].

Recently, a NMR spectroscopy method has been developed to estimate HDL cholesterol efflux capacity from serum [75. That method can form the basis of a genetic analysis of HDL cholesterol efflux capacity and may complement the results here. We believe more laboratorial and epidemiological research is needed to clarify the roles of HDL subfractions and particle size in cardiovascular diseases.

\section{References}

[1] Naomi J Rankin, David Preiss, Paul Welsh, Karl E V Burgess, Scott M Nelson, Debbie A Lawlor, and Naveed Sattar. The emergence of proton nuclear magnetic resonance metabolomics in the cardiovascular arena as viewed from a clinical perspective. Atherosclerosis, 237(1):287-300, nov 2014. ISSN 1879-1484 (Electronic). doi: 10.1016/j.atherosclerosis.2014.09.024.

[2] Samia Mora, James D Otvos, Nader Rifai, Robert S Rosenson, Julie E Buring, and Paul M Ridker. Lipoprotein particle profiles by nuclear magnetic resonance compared with standard lipids and apolipoproteins in predicting incident cardiovascular disease in women. Circulation, 119(7):931-939, feb 2009. ISSN 1524-4539 (Electronic). doi: 10.1161/CIRCULATIONAHA.108.816181.

[3] Michael V Holmes, Iona Y Millwood, Christiana Kartsonaki, Michael R Hill, Derrick A Bennett, Ruth Boxall, Yu Guo, Xin Xu, Zheng Bian, Ruying Hu, Robin G Walters, Junshi Chen, Mika Ala-Korpela, Sarah Parish, Robert J Clarke, Richard Peto, Rory Collins, Liming Li, and Zhengming Chen. Lipids, Lipoproteins, and Metabolites and Risk of Myocardial Infarction and Stroke. Journal of the American College of Cardiology, 71 (6):620-632, feb 2018. ISSN 1558-3597 (Electronic). doi: 10.1016/j.jacc.2017.12.006.

[4] Ron C Hoogeveen, John W Gaubatz, Wensheng Sun, Rhiannon C Dodge, Jacy R Crosby, Jennifer Jiang, David Couper, Salim S Virani, Sekar Kathiresan, Eric Boerwinkle, and Christie M Ballantyne. Small dense low-density lipoprotein-cholesterol concentrations predict risk for coronary heart disease: the Atherosclerosis 
Risk In Communities (ARIC) study. Arteriosclerosis, thrombosis, and vascular biology, 34(5):1069-1077, may 2014. ISSN 1524-4636 (Electronic). doi: 10.1161/ATVBAHA.114.303284.

[5] Paul T Williams, Xue-Qiao Zhao, Santica M Marcovina, James D Otvos, B Greg Brown, and Ronald M Krauss. Comparison of four methods of analysis of lipoprotein particle subfractions for their association with angiographic progression of coronary artery disease. Atherosclerosis, 233(2):713-720, apr 2014. ISSN 1879-1484 (Electronic). doi: 10.1016/j.atherosclerosis.2014.01.034.

[6] Chobufo Ditah, James Otvos, Hisham Nassar, Dorith Shaham, Ronit Sinnreich, and Jeremy D Kark. Small and medium sized HDL particles are protectively associated with coronary calcification in a cross-sectional population-based sample. Atherosclerosis, 251:124-131, aug 2016. ISSN 1879-1484 (Electronic). doi: 10.1016/ j.atherosclerosis.2016.06.010.

[7] Patrick R Lawler, Akintunde O Akinkuolie, Paulo Harada, Robert J Glynn, Daniel I Chasman, Paul M Ridker, and Samia Mora. Residual Risk of Atherosclerotic Cardiovascular Events in Relation to Reductions in VeryLow-Density Lipoproteins. Journal of the American Heart Association, 6(12), dec 2017. ISSN 2047-9980 (Electronic). doi: 10.1161/JAHA.117.007402.

[8] Krista Fischer, Johannes Kettunen, Peter Wurtz, Toomas Haller, Aki S Havulinna, Antti J Kangas, Pasi Soininen, Tonu Esko, Mari-Liis Tammesoo, Reedik Magi, Steven Smit, Aarno Palotie, Samuli Ripatti, Veikko Salomaa, Mika Ala-Korpela, Markus Perola, and Andres Metspalu. Biomarker profiling by nuclear magnetic resonance spectroscopy for the prediction of all-cause mortality: an observational study of 17,345 persons. PLoS medicine, 11(2):e1001606, feb 2014. ISSN 1549-1676 (Electronic). doi: 10.1371/journal.pmed.1001606.

[9] Peter Wurtz, Juho R Raiko, Costan G Magnussen, Pasi Soininen, Antti J Kangas, Tuulia Tynkkynen, Russell Thomson, Reino Laatikainen, Markku J Savolainen, Jari Laurikka, Pekka Kuukasjarvi, Matti Tarkka, Pekka J Karhunen, Antti Jula, Jorma S Viikari, Mika Kahonen, Terho Lehtimaki, Markus Juonala, Mika Ala-Korpela, and Olli T Raitakari. High-throughput quantification of circulating metabolites improves prediction of subclinical atherosclerosis. European heart journal, 33(18):2307-2316, sep 2012. ISSN 1522-9645 (Electronic). doi: 10.1093/eurheartj/ehs020.

[10] Daniel B van Schalkwijk, Albert A de Graaf, Evgeni Tsivtsivadze, Laurence D Parnell, Bianca J C van der Werff-van der Vat, Ben van Ommen, Jan van der Greef, and Jose M Ordovas. Lipoprotein metabolism indicators improve cardiovascular risk prediction. PloS one, 9(3):e92840, 2014. ISSN 1932-6203 (Electronic). doi: $10.1371 /$ journal.pone.0092840.

[11] Robert W McGarrah, Damian M Craig, Carol Haynes, Z Elaine Dowdy, Svati H Shah, and William E Kraus. High-density lipoprotein subclass measurements improve mortality risk prediction, discrimination and reclassification in a cardiac catheterization cohort. Atherosclerosis, 246:229-235, mar 2016. ISSN 1879-1484 (Electronic). doi: 10.1016/j.atherosclerosis.2016.01.012.

[12] B Lamarche, A Tchernof, S Moorjani, B Cantin, G R Dagenais, P J Lupien, and J P Despres. Small, dense low-density lipoprotein particles as a predictor of the risk of ischemic heart disease in men. Prospective results from the Quebec Cardiovascular Study. Circulation, 95(1):69-75, jan 1997. ISSN 0009-7322 (Print).

[13] H Campos, L A Moye, S P Glasser, M J Stampfer, and F M Sacks. Low-density lipoprotein size, pravastatin treatment, and coronary events. JAMA, 286(12):1468-1474, sep 2001. ISSN 0098-7484 (Print).

[14] Samia Mora. Advanced lipoprotein testing and subfractionation are not (yet) ready for routine clinical use. Circulation, 119(17):2396-2404, may 2009. ISSN 1524-4539 (Electronic). doi: 10.1161/CIRCULATIONAHA. 108.819359 .

[15] Daniel Seung Kim, Yatong K Li, Griffith A Bell, Amber A Burt, Tomas Vaisar, Patrick M Hutchins, Clement E Furlong, James D Otvos, Joseph F Polak, Martinson Kweku Arnan, Joel D Kaufman, Robyn L McClelland, W T Jr Longstreth, and Gail P Jarvik. Concentration of Smaller High-Density Lipoprotein Particle (HDL-P) Is Inversely Correlated With Carotid Intima Media Thickening After Confounder Adjustment: The Multi Ethnic Study of Atherosclerosis (MESA). Journal of the American Heart Association, 5(5), may 2016. ISSN 2047-9980 (Electronic). doi: 10.1161/JAHA.115.002977. 
[16] Gunther Silbernagel, Philipp Pagel, Volker Pfahlert, Bernd Genser, Hubert Scharnagl, Marcus E Kleber, Graciela Delgado, Haruna Ohrui, Andreas Ritsch, Tanja B Grammer, Wolfgang Koenig, and Winfried Marz. High-Density Lipoprotein Subclasses, Coronary Artery Disease, and Cardiovascular Mortality. Clinical chemistry, 63(12):1886-1896, dec 2017. ISSN 1530-8561 (Electronic). doi: 10.1373/clinchem.2017.275636.

[17] Jian-Jun Li, Yan Zhang, Sha Li, Chuan-Jue Cui, Cheng-Gang Zhu, Yuan-Lin Guo, Na-Qiong Wu, Rui-Xia Xu, Geng Liu, Qian Dong, and Jing Sun. Large HDL Subfraction But Not HDL-C Is Closely Linked With Risk Factors, Coronary Severity and Outcomes in a Cohort of Nontreated Patients With Stable Coronary Artery Disease: A Prospective Observational Study. Medicine, 95(4):e2600, jan 2016. ISSN 1536-5964 (Electronic). doi: 10.1097/MD.0000000000002600.

[18] Benoit J Arsenault, Isabelle Lemieux, Jean-Pierre Despres, Pascale Gagnon, Nicholas J Wareham, Erik S G Stroes, John J P Kastelein, Kay-Tee Khaw, and S Matthijs Boekholdt. HDL particle size and the risk of coronary heart disease in apparently healthy men and women: the EPIC-Norfolk prospective population study. Atherosclerosis, 206(1):276-281, sep 2009. ISSN 1879-1484 (Electronic). doi: 10.1016/j.atherosclerosis. 2009.01.044.

[19] H Robert Superko. Advanced lipoprotein testing and subfractionation are clinically useful. Circulation, 119 (17):2383-2395, may 2009. ISSN 1524-4539 (Electronic). doi: 10.1161/CIRCULATIONAHA.108.809582.

[20] Michael H Davidson, Christie M Ballantyne, Terry A Jacobson, Vera A Bittner, Lynne T Braun, Alan S Brown, W Virgil Brown, William C Cromwell, Ronald B Goldberg, James M McKenney, Alan T Remaley, Allan D Sniderman, Peter P Toth, Sotirios Tsimikas, Paul E Ziajka, Kevin C Maki, and Mary R Dicklin. Clinical utility of inflammatory markers and advanced lipoprotein testing: advice from an expert panel of lipid specialists. Journal of clinical lipidology, 5(5):338-367, 2011. ISSN 1933-2874 (Print). doi: 10.1016/j.jacl.2011.07.005.

[21] Harold E Bays, Peter H Jones, Carl E Orringer, W Virgil Brown, and Terry A Jacobson. National Lipid Association Annual Summary of Clinical Lipidology 2016. Journal of clinical lipidology, 10(1 Suppl):S1-43, 2016. ISSN 1933-2874 (Print). doi: 10.1016/j.jacl.2015.08.002.

[22] George Davey Smith and Shah Ebrahim. 'Mendelian randomization': Can genetic epidemiology contribute to understanding environmental determinants of disease? International Journal of Epidemiology, 32(1):1-22, feb 2003. ISSN 03005771. doi: 10.1093/ije/dyg070.

[23] Vanessa Didelez and Nuala Sheehan. Mendelian randomization as an instrumental variable approach to causal inference. Statistical Methods in Medical Research, 16(4):309-330, 2007.

[24] George Davey Smith and Gibran Hemani. Mendelian randomization: genetic anchors for causal inference in epidemiological studies. Human Molecular Genetics, 23(R1):R89-R98, 2014.

[25] Samuel S Gidding, Stephen R Daniels, and Rae Ellen W Kavey. Developing the 2011 Integrated Pediatric Guidelines for Cardiovascular Risk Reduction. Pediatrics, 129(5):e1311-9, may 2012. ISSN 1098-4275 (Electronic). doi: 10.1542/peds.2011-2903.

[26] Neil M Davies, Michael V Holmes, and George Davey Smith. Reading Mendelian randomisation studies: a guide, glossary, and checklist for clinicians. BMJ (Clinical research ed.), 362:k601, jul 2018. ISSN 1756-1833 (Electronic). doi: 10.1136/bmj.k601.

[27] Connor A Emdin, Amit V Khera, Pradeep Natarajan, Derek Klarin, Hong-Hee Won, Gina M Peloso, Nathan O Stitziel, Akihiro Nomura, Seyedeh M Zekavat, Alexander G Bick, Namrata Gupta, Rosanna Asselta, Stefano Duga, Piera Angelica Merlini, Adolfo Correa, Thorsten Kessler, James G Wilson, Matthew J Bown, Alistair S Hall, Peter S Braund, Nilesh J Samani, Heribert Schunkert, Jaume Marrugat, Roberto Elosua, Ruth McPherson, Martin Farrall, Hugh Watkins, Cristen Willer, Goncalo R Abecasis, Janine F Felix, Ramachandran S Vasan, Eric Lander, Daniel J Rader, John Danesh, Diego Ardissino, Stacey Gabriel, Danish Saleheen, and Sekar Kathiresan. Phenotypic Characterization of Genetically Lowered Human Lipoprotein(a) Levels. Journal of the American College of Cardiology, 68(25):2761-2772, dec 2016. ISSN 1558-3597 (Electronic). doi: 10.1016/j.jacc.2016.10.033.

[28] Brian A Ference, John J P Kastelein, Henry N Ginsberg, M John Chapman, Stephen J Nicholls, Kausik K Ray, Chris J Packard, Ulrich Laufs, Robert D Brook, Clare Oliver-Williams, Adam S Butterworth, John Danesh, George Davey Smith, Alberico L Catapano, and Marc S Sabatine. Association of Genetic Variants Related to 
CETP Inhibitors and Statins With Lipoprotein Levels and Cardiovascular Risk. JAMA, 318(10):947-956, sep 2017. ISSN 1538-3598 (Electronic). doi: 10.1001/jama.2017.11467.

[29] Thomas J Hoffmann, Elizabeth Theusch, Tanushree Haldar, Dilrini K Ranatunga, Eric Jorgenson, Marisa W Medina, Mark N Kvale, Pui-Yan Kwok, Catherine Schaefer, Ronald M Krauss, Carlos Iribarren, and Neil Risch. A large electronic-health-record-based genome-wide study of serum lipids. Nature genetics, 50(3): 401-413, mar 2018. ISSN 1546-1718 (Electronic). doi: 10.1038/s41588-018-0064-5.

[30] Cristen J Willer, Ellen M Schmidt, Sebanti Sengupta, Gina M Peloso, Stefan Gustafsson, Stavroula Kanoni, Andrea Ganna, Jin Chen, Martin L Buchkovich, Samia Mora, Jacques S Beckmann, Jennifer L Bragg-Gresham, Hsing-Yi Chang, Ayse Demirkan, Heleen M Den Hertog, Ron Do, Louise A Donnelly, Georg B Ehret, Tonu Esko, Mary F Feitosa, Teresa Ferreira, Krista Fischer, Pierre Fontanillas, Ross M Fraser, Daniel F Freitag, Deepti Gurdasani, Kauko Heikkila, Elina Hypponen, Aaron Isaacs, Anne U Jackson, Asa Johansson, Toby Johnson, Marika Kaakinen, Johannes Kettunen, Marcus E Kleber, Xiaohui Li, Jian'an Luan, Leo-Pekka Lyytikainen, Patrik K E Magnusson, Massimo Mangino, Evelin Mihailov, May E Montasser, Martina MullerNurasyid, Ilja M Nolte, Jeffrey R O'Connell, Colin N A Cameron D Palmer, Markus Perola, Ann-Kristin Petersen, Serena Sanna, Richa Saxena, Susan K Service, Sonia Shah, Dmitry Shungin, Carlo Sidore, Ci Song, Rona J Strawbridge, Ida Surakka, Toshiko Tanaka, Tanya M Teslovich, Gudmar Thorleifsson, Evita G Van den Herik, Benjamin F Voight, Kelly A Volcik, Lindsay L Waite, Andrew Wong, Ying Wu, Weihua Zhang, Devin Absher, Gershim Asiki, Ines Barroso, Latonya F Been, Jennifer L Bolton, Lori L Bonnycastle, Paolo Brambilla, Mary S Burnett, Giancarlo Cesana, Maria Dimitriou, Alex S F Doney, Angela Doring, Paul Elliott, Stephen E Epstein, Gudmundur Ingi Eyjolfsson, Bruna Gigante, Mark O Goodarzi, Harald Grallert, Martha L Gravito, Christopher J Groves, Goran Hallmans, Anna-Liisa Hartikainen, Caroline Hayward, Dena Hernandez, Andrew A Hicks, Hilma Holm, Yi-Jen Hung, Thomas Illig, Michelle R Jones, Pontiano Kaleebu, John J P Kastelein, Kay-Tee Khaw, Eric Kim, Norman Klopp, Pirjo Komulainen, Meena Kumari, Claudia Langenberg, Terho Lehtimaki, Shih-Yi Lin, Jaana Lindstrom, Ruth J F Loos, Francois Mach, Wendy L McArdle, Christa Meisinger, Braxton D Mitchell, Gabrielle Muller, Ramaiah Nagaraja, Narisu Narisu, Tuomo V M Nieminen, Rebecca N Nsubuga, Isleifur Olafsson, Ken K Ong, Aarno Palotie, Theodore Papamarkou, Cristina Pomilla, Anneli Pouta, Daniel J Rader, Muredach P Reilly, Paul M Ridker, Fernando Rivadeneira, Igor Rudan, Aimo Ruokonen, Nilesh Samani, Hubert Scharnagl, Janet Seeley, Kaisa Silander, Alena Stancakova, Kathleen Stirrups, Amy J Swift, Laurence Tiret, Andre G Uitterlinden, L Joost van Pelt, Sailaja Vedantam, Nicholas Wainwright, Cisca Wijmenga, Sarah H Wild, Gonneke Willemsen, Tom Wilsgaard, James F Wilson, Elizabeth H Young, Jing Hua Zhao, Linda S Adair, Dominique Arveiler, Themistocles L Assimes, Stefania Bandinelli, Franklyn Bennett, Murielle Bochud, Bernhard O Boehm, Dorret I Boomsma, Ingrid B Borecki, Stefan R Bornstein, Pascal Bovet, Michel Burnier, Harry Campbell, Aravinda Chakravarti, John C Chambers, Yii-Der Ida Chen, Francis S Collins, Richard S Cooper, John Danesh, George Dedoussis, Ulf de Faire, Alan B Feranil, Jean Ferrieres, Luigi Ferrucci, Nelson B Freimer, Christian Gieger, Leif C Groop, Vilmundur Gudnason, Ulf Gyllensten, Anders Hamsten, Tamara B Harris, Aroon Hingorani, Joel N Hirschhorn, Albert Hofman, G Kees Hovingh, Chao Agnes Hsiung, Steve E Humphries, Steven C Hunt, Kristian Hveem, Carlos Iribarren, Marjo-Riitta Jarvelin, Antti Jula, Mika Kahonen, Jaakko Kaprio, Antero Kesaniemi, Mika Kivimaki, Jaspal S Kooner, Peter J Koudstaal, Ronald M Krauss, Diana Kuh, Johanna Kuusisto, Kirsten O Kyvik, Markku Laakso, Timo A Lakka, Lars Lind, Cecilia M Lindgren, Nicholas G Martin, Winfried Marz, Mark I McCarthy, Colin A McKenzie, Pierre Meneton, Andres Metspalu, Leena Moilanen, Andrew D Morris, Patricia B Munroe, Inger Njolstad, Nancy L Pedersen, Chris Power, Peter P Pramstaller, Jackie F Price, Bruce M Psaty, Thomas Quertermous, Rainer Rauramaa, Danish Saleheen, Veikko Salomaa, Dharambir K Sanghera, Jouko Saramies, Peter E H Schwarz, Wayne H-H Sheu, Alan R Shuldiner, Agneta Siegbahn, Tim D Spector, Kari Stefansson, David P Strachan, Bamidele O Tayo, Elena Tremoli, Jaakko Tuomilehto, Matti Uusitupa, Cornelia M van Duijn, Peter Vollenweider, Lars Wallentin, Nicholas J Wareham, John B Whitfield, Bruce H R Wolffenbuttel, Jose M Ordovas, Eric Boerwinkle, Colin N A Cameron D Palmer, Unnur Thorsteinsdottir, Daniel I Chasman, Jerome I Rotter, Paul W Franks, Samuli Ripatti, L Adrienne Cupples, Manjinder S Sandhu, Stephen S Rich, Michael Boehnke, Panos Deloukas, Sekar Kathiresan, Karen L Mohlke, Erik Ingelsson, Goncalo R Abecasis, and Global Lipids Genetics Consortium. Discovery and refinement of loci associated with lipid levels. Nature Genetics, 45(11):1274, nov 2013. ISSN 1546-1718 (Electronic). doi: 10.1038/ng.2797.

[31] James P Davis, Jeroen R Huyghe, Adam E Locke, Anne U Jackson, Xueling Sim, Heather M Stringham, Tanya M Teslovich, Ryan P Welch, Christian Fuchsberger, Narisu Narisu, Peter S Chines, Antti J Kangas, Pasi Soininen, Mika Ala-Korpela, Johanna Kuusisto, Francis S Collins, Markku Laakso, Michael Boehnke, and Karen L Mohlke. Common, low-frequency, and rare genetic variants associated with lipoprotein subclasses 
and triglyceride measures in Finnish men from the METSIM study. PLoS genetics, 13(10):e1007079, oct 2017. ISSN 1553-7404 (Electronic). doi: 10.1371/journal.pgen.1007079.

[32] Johannes Kettunen, Ayse Demirkan, Peter Wurtz, Harmen H M Draisma, Toomas Haller, Rajesh Rawal, Anika Vaarhorst, Antti J Kangas, Leo-Pekka Lyytikainen, Matti Pirinen, Rene Pool, Antti-Pekka Sarin, Pasi Soininen, Taru Tukiainen, Qin Wang, Mika Tiainen, Tuulia Tynkkynen, Najaf Amin, Tanja Zeller, Marian Beekman, Joris Deelen, Ko Willems van Dijk, Tonu Esko, Jouke-Jan Hottenga, Elisabeth M van Leeuwen, Terho Lehtimaki, Evelin Mihailov, Richard J Rose, Anton J M de Craen, Christian Gieger, Mika Kahonen, Markus Perola, Stefan Blankenberg, Markku J Savolainen, Aswin Verhoeven, Jorma Viikari, Gonneke Willemsen, Dorret I Boomsma, Cornelia M van Duijn, Johan Eriksson, Antti Jula, Marjo-Riitta Jarvelin, Jaakko Kaprio, Andres Metspalu, Olli Raitakari, Veikko Salomaa, P Eline Slagboom, Melanie Waldenberger, Samuli Ripatti, and Mika Ala-Korpela. Genome-wide study for circulating metabolites identifies 62 loci and reveals novel systemic effects of LPA. Nature communications, 7:11122, mar 2016. ISSN 2041-1723 (Electronic). doi: $10.1038 /$ ncomms11122.

[33] The CARDIoGRAMplusC4D Consortium, Majid Nikpay, Anuj Goel, Hong-Hee Won, Leanne M Hall, Christina Willenborg, Stavroula Kanoni, Danish Saleheen, Theodosios Kyriakou, Christopher P Nelson, Jemma C Hopewell, Others, Thomas R Webb, Lingyao Zeng, Abbas Dehghan, Maris Alver, Sebastian M Armasu, Kirsi Auro, Andrew Bjonnes, Daniel I Chasman, Shufeng Chen, Ian Ford, Nora Franceschini, Christian Gieger, Christopher Grace, Stefan Gustafsson, Jie Jianfeng Huang, Shih-Jen Hwang, Yun Kyoung Kim, Marcus E Kleber, King Wai Lau, Xiangfeng Lu, Yingchang Lu, Leo-Pekka Lyytikainen, Evelin Mihailov, Alanna C Morrison, Natalia Pervjakova, Liming Qu, Lynda M Rose, Elias Salfati, Richa Saxena, Markus Scholz, Albert V Smith, Emmi Tikkanen, Andre Uitterlinden, Xueli Yang, Weihua Zhang, Wei Zhao, Mariza de Andrade, Paul S de Vries, Natalie R van Zuydam, Sonia S Anand, Lars Bertram, Frank Beutner, George Dedoussis, Philippe Frossard, Dominique Gauguier, Alison H Goodall, Omri Gottesman, Marc Haber, Bok-Ghee Han, Jie Jianfeng Huang, Shapour Jalilzadeh, Thorsten Kessler, Inke R Konig, Lars Lannfelt, Wolfgang Lieb, Lars Lind, Cecilia M Lindgren, Marja-Liisa Lokki, Patrik K Magnusson, Nadeem H Mallick, Narinder Mehra, Thomas Meitinger, Fazal-Ur-Rehman Memon, Andrew P Morris, Markku S Nieminen, Nancy L Pedersen, Annette Peters, Loukianos S Rallidis, Asif Rasheed, Maria Samuel, Svati H Shah, Juha Sinisalo, Kathleen E Stirrups, Stella Trompet, Laiyuan Wang, Khan S Zaman, Diego Ardissino, Eric Boerwinkle, Ingrid B Borecki, Erwin P Bottinger, Julie E Buring, John C Chambers, Rory Collins, L Adrienne Cupples, John Danesh, Ilja Demuth, Roberto Elosua, Stephen E Epstein, Tonu Esko, Mary F Feitosa, Oscar H Franco, Maria Grazia Franzosi, Christopher B Granger, Dongfeng Gu, Vilmundur Gudnason, Alistair S Hall, Anders Hamsten, Tamara B Harris, Stanley L Hazen, Christian Hengstenberg, Albert Hofman, Erik Ingelsson, Carlos Iribarren, J Wouter Jukema, Pekka J Karhunen, Bong-Jo Kim, Jaspal S Kooner, Iftikhar J Kullo, Terho Lehtimaki, Ruth J F Loos, Olle Melander, Andres Metspalu, Winfried Marz, Colin N Palmer, Markus Perola, Thomas Quertermous, Daniel J Rader, Paul M Ridker, Samuli Ripatti, Robert Roberts, Veikko Salomaa, Dharambir K Sanghera, Stephen M Schwartz, Udo Seedorf, Alexandre F Stewart, David J Stott, Joachim Thiery, Pierre A Zalloua, Christopher J O'Donnell, Muredach P Reilly, Themistocles L Assimes, John R Thompson, Jeanette Erdmann, Robert Clarke, Hugh Watkins, Sekar Kathiresan, Ruth McPherson, Panos Deloukas, Heribert Schunkert, Nilesh J Samani, and Martin Farrall. A comprehensive 1000 Genomes-based genome-wide association meta-analysis of coronary artery disease. Nature Genetics, 47(10):1121, oct 2015. ISSN 1546-1718 (Electronic). doi: 10.1038/ng.3396.

[34] Christopher P Nelson, Anuj Goel, Adam S Butterworth, Stavroula Kanoni, Tom R Webb, Eirini Marouli, Lingyao Zeng, Ioanna Ntalla, Florence Y Lai, Jemma C Hopewell, Olga Giannakopoulou, Tao Jiang, Stephen E Hamby, Emanuele Di Angelantonio, Themistocles L Assimes, Erwin P Bottinger, John C Chambers, Robert Clarke, Colin N A Palmer, Richard M Cubbon, Patrick Ellinor, Raili Ermel, Evangelos Evangelou, Paul W Franks, Christopher Grace, Dongfeng Gu, Aroon D Hingorani, Joanna M M Howson, Erik Ingelsson, Adnan Kastrati, Thorsten Kessler, Theodosios Kyriakou, Terho Lehtimaki, Xiangfeng Lu, Yingchang Lu, Winfried Marz, Ruth McPherson, Andres Metspalu, Mar Pujades-Rodriguez, Arno Ruusalepp, Eric E Schadt, Amand F Schmidt, Michael J Sweeting, Pierre A Zalloua, Kamal AlGhalayini, Bernard D Keavney, Jaspal S Kooner, Ruth J F Loos, Riyaz S Patel, Martin K Rutter, Maciej Tomaszewski, Ioanna Tzoulaki, Eleftheria Zeggini, Jeanette Erdmann, George Dedoussis, Johan L M Bjorkegren, Heribert Schunkert, Martin Farrall, John Danesh, Nilesh J Samani, Hugh Watkins, and Panos Deloukas. Association analyses based on false discovery rate implicate new loci for coronary artery disease. Nature genetics, 49(9):1385-1391, sep 2017. ISSN 1546-1718 (Electronic). doi: 10.1038/ng.3913. 
[35] Liam Abbott, Sam Bryant, Claire Churchhouse, Andrea Ganna, Daniel Howrigan, Duncan Palmer, Ben Neale, Raymond Walters, Caitlin Carey, and The Hail team. Round $2\{$ GWAS $\}$ results of thousands of phenotype in the $\{\mathrm{UK}\}\{$ BioBank $\}, 2018$. URL http://www.nealelab.is/uk-biobank/.

[36] Pasi Soininen, Antti J Kangas, Peter Wurtz, Taru Tukiainen, Tuulia Tynkkynen, Reino Laatikainen, MarjoRiitta Jarvelin, Mika Kahonen, Terho Lehtimaki, Jorma Viikari, Olli T Raitakari, Markku J Savolainen, and Mika Ala-Korpela. High-throughput serum NMR metabonomics for cost-effective holistic studies on systemic metabolism. The Analyst, 134(9):1781-1785, sep 2009. ISSN 1364-5528 (Electronic). doi: 10.1039/b910205a.

[37] Brendan K Bulik-Sullivan, Po-Ru Loh, Hilary K Finucane, Stephan Ripke, Jian Yang, Nick Patterson, Mark J Daly, Alkes L Price, and Benjamin M Neale. $\{\mathrm{LD}\}$ score regression distinguishes confounding from polygenicity in genome-wide association studies. Nature Genetics, 47(3):291, 2015.

[38] Qingyuan Zhao, Yang Chen, Jingshu Wang, and Dylan S Small. Powerful three-sample genome-wide design and robust statistical inference in summary-data Mendelian randomization. International Journal of Epidemiology, 48(5):1478-1492, oct 2019. ISSN 1464-3685 (Electronic). doi: 10.1093/ije/dyz142.

[39] Brandon L Pierce and Stephen Burgess. Efficient design for Mendelian randomization studies: subsample and 2-sample instrumental variable estimators. American journal of epidemiology, 178(7):1177-1184, oct 2013. ISSN 1476-6256 (Electronic). doi: 10.1093/aje/kwt084.

[40] Gibran Hemani, Jie Zheng, Kaitlin H Wade, Charles Laurin, Benjamin Elsworth, Stephen Burgess, Jack Bowden, Ryan Langdon, Vanessa Tan, James Yarmolinsky, Hashem A Shihab, Nicholas Timpson, David M Evans, Caroline Relton, Richard M Martin, George Davey Smith, Tom R Gaunt, Philip C Haycock, and The MR-Base Collaboration. MR-Base: a platform for systematic causal inference across the phenome using billions of genetic associations. bioRxiv:078972, 2016.

[41] Shaun Purcell, Benjamin Neale, Kathe Todd-Brown, Lori Thomas, Manuel A R Ferreira, David Bender, Julian Maller, Pamela Sklar, Paul I W De Bakker, Mark J Daly, and Pak C Sham. \{PLINK\}: a tool set for whole-genome association and population-based linkage analyses. American Journal of Human Genetics, 81 (3):559-575, 2007.

[42] Tom G Richardson, Eleanor Sanderson, Tom M Palmer, Mika Ala-Korpela, Brian A Ference, George Davey Smith, and Michael V Holmes. Evaluating the relationship between circulating lipoprotein lipids and apolipoproteins with risk of coronary heart disease: A multivariable Mendelian randomisation analysis. PLoS medicine, 17(3):e1003062, mar 2020. ISSN 1549-1676 (Electronic). doi: 10.1371/journal.pmed.1003062.

[43] Qingyuan Zhao, Jingshu Wang, Gibran Hemani, Jack Bowden, and Dylan S. Small. Statistical inference in two-sample summary-data mendelian randomization using robust adjusted profile score. Annals of Statistics, 48(3):1742-1769, 2020. doi: 10.1214/19-aos1866. URL https://doi.org/10.1214/19-aos1866.

[44] Jingshu Wang, Qingyuan Zhao, Jack Bowden, Gibran Hemani, George Davey Smith, Dylan S Small, and Nancy R Zhang. Causal Inference for Heritable Phenotypic Risk Factors Using Heterogeneous Genetic Instruments. bioRxiv:077982, 2020.

[45] Eleanor Sanderson, George Davey Smith, Frank Windmeijer, and Jack Bowden. An examination of multivariable Mendelian randomization in the single-sample and two-sample summary data settings. International Journal of Epidemiology, 48(3):713-727, jun 2019. ISSN 0300-5771. doi: 10.1093/ije/dyy262. URL https://academic.oup.com/ije/article/48/3/713/5238110

[46] Yoav Benjamini and Yosef Hochberg. Controlling the False Discovery Rate: A Practical and Powerful Approach to Multiple Testing. Journal of the Royal Statistical Society: Series B (Methodological), 57(1):289-300, jan 1995. ISSN 0035-9246. doi: https://doi.org/10.1111/j.2517-6161.1995.tb02031.x. URL https://doi.org/10. $1111 / \mathrm{j} .2517-6161.1995 . \mathrm{tb02031.x}$

[47] Stephen Burgess, Adam Butterworth, and Simon G Thompson. Mendelian randomization analysis with multiple genetic variants using summarized data. Genetic Epidemiology, 37(7):658-665, 2013.

[48] Jack Bowden, George Davey Smith, Philip C Haycock, and Stephen Burgess. Consistent estimation in Mendelian randomization with some invalid instruments using a weighted median estimator. Genetic Epidemiology, 40(4):304-314, 2016. 
[49] Michael V Holmes, Folkert W Asselbergs, Tom M Palmer, Fotios Drenos, Matthew B Lanktree, Christopher P Nelson, Caroline E Dale, Sandosh Padmanabhan, Chris Finan, Daniel I Swerdlow, and Others. Mendelian randomization of blood lipids for coronary heart disease. European Heart Journal, 36(9):539-550, 2014.

[50] Jack Bowden, George Davey Smith, and Stephen Burgess. Mendelian randomization with invalid instruments: effect estimation and bias detection through Egger regression. International Journal of Epidemiology, 44(2): $512-525,2015$.

[51] Philip J Barter, Mark Caulfield, Mats Eriksson, Scott M Grundy, John J P Kastelein, Michel Komajda, Jose Lopez-Sendon, Lori Mosca, Jean-Claude Tardif, David D Waters, and Others. Effects of torcetrapib in patients at high risk for coronary events. New England Journal of Medicine, 357(21):2109-2122, 2007.

[52] Gregory G Schwartz, Anders G Olsson, Markus Abt, Christie M Ballantyne, Philip J Barter, Jochen Brumm, Bernard R Chaitman, Ingar M Holme, David Kallend, Lawrence A Leiter, and Others. Effects of dalcetrapib in patients with a recent acute coronary syndrome. New England Journal of Medicine, 367(22):2089-2099, 2012 .

[53] A Michael Lincoff, Stephen J Nicholls, Jeffrey S Riesmeyer, Philip J Barter, H Bryan Brewer, Keith A A Fox, C Michael Gibson, Christopher Granger, Venu Menon, Gilles Montalescot, and Others. Evacetrapib and cardiovascular outcomes in high-risk vascular disease. New England Journal of Medicine, 376(20):1933-1942, 2017.

[54] Stephen J Nicholls, Rishi Puri, Christie M Ballantyne, J Wouter Jukema, John J P Kastelein, Wolfgang Koenig, R Scott Wright, David Kallend, Peter Wijngaard, Marilyn Borgman, Kathy Wolski, and Steven E Nissen. Effect of Infusion of High-Density Lipoprotein Mimetic Containing Recombinant Apolipoprotein A-I Milano on Coronary Disease in Patients With an Acute Coronary Syndrome in the MILANO-PILOT Trial: A Randomized Clinical Trial. JAMA cardiology, 3(9):806-814, sep 2018. ISSN 2380-6591 (Electronic). doi: 10.1001/jamacardio.2018.2112.

[55] G J Miller and N E Miller. Plasma-high-density-lipoprotein concentration and development of ischaemic heart-disease. Lancet, 305(7897):16-19, 1975.

[56] Prospective Studies Collaboration. Blood cholesterol and vascular mortality by age, sex, and blood pressure: a meta-analysis of individual data from 61 prospective studies with 55,000 vascular deaths. Lancet, 370(9602): 1829-1839, 2007.

[57] Emerging Risk Factors Collaboration. Major lipids, apolipoproteins, and risk of vascular disease. JAMA, 302 (18):1993-2000, 2009.

[58] Benjamin F Voight, Gina M Peloso, Marju Orho-Melander, Ruth Frikke-Schmidt, Maja Barbalic, Majken K Jensen, George Hindy, Hilma Hólm, Eric L Ding, Toby Johnson, and Others. Plasma \{HDL\} cholesterol and risk of myocardial infarction: a mendelian randomisation study. Lancet, 380(9841):572-580, 2012.

[59] Michael V Holmes, Mika Ala-Korpela, and George Davey Smith. Mendelian randomization in cardiometabolic disease: challenges in evaluating causality. Nature Reviews Cardiology, 14(10):577, 2017.

[60] Jon White, Daniel I Swerdlow, David Preiss, Zammy Fairhurst-Hunter, Brendan J Keating, Folkert W Asselbergs, Naveed Sattar, Steve E Humphries, Aroon D Hingorani, and Michael V Holmes. Association of lipid fractions with risks for coronary artery disease and diabetes. JAMA Cardiology, 1(6):692-699, 2016.

[61] Daniel J Rader and G Kees Hovingh. HDL and cardiovascular disease. Lancet, 384(9943):618-625, 2014.

[62] Anand Rohatgi, Amit Khera, Jarett D Berry, Edward G Givens, Colby R Ayers, Kyle E Wedin, Ian J Neeland, Ivan S Yuhanna, Daniel R Rader, James A de Lemos, and Others. HDL cholesterol efflux capacity and incident cardiovascular events. New England Journal of Medicine, 371(25):2383-2393, 2014.

[63] Danish Saleheen, Robert Scott, Sundas Javad, Wei Zhao, Amrith Rodrigues, Antonino Picataggi, Daniya Lukmanova, Megan L Mucksavage, Robert Luben, Jeffery Billheimer, John J P Kastelein, S Matthijs Boekholdt, Kay-Tee Khaw, Nick Wareham, and Daniel J Rader. Association of HDL cholesterol efflux capacity with incident coronary heart disease events: a prospective case-control study. The lancet. Diabetes $\&$ endocrinology, 3(7):507-513, jul 2015. ISSN 2213-8595 (Electronic). doi: 10.1016/S2213-8587(15)00126-6. 
[64] Samar R El Khoudary, Patrick M Hutchins, Karen A Matthews, Maria M Brooks, Trevor J Orchard, Graziella E Ronsein, and Jay W Heinecke. Cholesterol Efflux Capacity and Subclasses of HDL Particles in Healthy Women Transitioning Through Menopause. The Journal of clinical endocrinology and metabolism, 101(9):3419-3428, sep 2016. ISSN 1945-7197 (Electronic). doi: 10.1210/jc.2016-2144.

[65] R Kannan Mutharasan, C Shad Thaxton, Jarett Berry, Martha L Daviglus, Chun Yuan, Jie Sun, Colby Ayers, Donald M Lloyd-Jones, and John T Wilkins. HDL efflux capacity, HDL particle size, and high-risk carotid atherosclerosis in a cohort of asymptomatic older adults: the Chicago Healthy Aging Study. Journal of lipid research, 58(3):600-606, mar 2017. ISSN 1539-7262 (Electronic). doi: 10.1194/jlr.P069039.

[66] Elda Favari, Laura Calabresi, Maria Pia Adorni, Wendy Jessup, Sara Simonelli, Guido Franceschini, and Franco Bernini. Small discoidal pre-beta1 HDL particles are efficient acceptors of cell cholesterol via ABCA1 and ABCG1. Biochemistry, 48(46):11067-11074, nov 2009. ISSN 1520-4995 (Electronic). doi: 10.1021/bi901564g.

[67] Xian-Ming Du, Mi-Jurng Kim, Liming Hou, Wilfried Le Goff, M John Chapman, Miranda Van Eck, Linda K Curtiss, John R Burnett, Sian P Cartland, Carmel M Quinn, Maaike Kockx, Anatol Kontush, Kerry-Anne Rye, Leonard Kritharides, and Wendy Jessup. HDL particle size is a critical determinant of ABCA1-mediated macrophage cellular cholesterol export. Circulation research, 116(7):1133-1142, mar 2015. ISSN 1524-4571 (Electronic). doi: 10.1161/CIRCRESAHA.116.305485.

[68] Paolo Zanoni, Sumeet A. Khetarpal, Daniel B. Larach, William F. Hancock-Cerutti, John S. Millar, Marina Cuchel, Stephanie DerOhannessian, Anatol Kontush, Praveen Surendran, Danish Saleheen, Stella Trompet, J. Wouter Jukema, Anton De Craen, Panos Deloukas, Naveed Sattar, Ian Ford, Chris Packard, Abdullah al Shafi Majumder, Dewan S. Alam, Emanuele Di Angelantonio, Goncalo Abecasis, Rajiv Chowdhury, Jeanette Erdmann, Børge G. Nordestgaard, Sune F. Nielsen, Anne Tybjærg-Hansen, Ruth Frikke Schmidt, Kari Kuulasmaa, Dajiang J. Liu, Markus Perola, Stefan Blankenberg, Veikko Salomaa, Satu Männistö, Philippe Amouyel, Dominique Arveiler, Jean Ferrieres, Martina Müller-Nurasyid, Marco Ferrario, Frank Kee, Cristen J. Willer, Nilesh Samani, Heribert Schunkert, Adam S. Butterworth, Joanna M. M. Howson, Gina M. Peloso, Nathan O. Stitziel, John Danesh, Sekar Kathiresan, and Daniel J. Rader. Rare variant in scavenger receptor BI raises HDL cholesterol and increases risk of coronary heart disease. Science, 351(6278):1166-1171, mar 2016. ISSN 0036-8075. doi: 10.1126/science.aad3517. URL https://www.sciencemag.org/lookup/doi/10.1126/science.aad3517.

[69] Sara Samadi, Zahra Farjami, Zeinab Sadat Hosseini, Gordon A. Ferns, Amir hooshang Mohammadpour, Maryam Tayefi, Homa Fal-Soleiman, Mohsen Moohebati, Majid Ghayour-Mobarhan, Habibollah Esmaily, and Amir Avan. Rare P376L variant in the SR-BI gene associates with HDL dysfunction and risk of cardiovascular disease. Clinical Biochemistry, 73:44-49, nov 2019. ISSN 00099120. doi: 10.1016/j.clinbiochem.2019.06.014. URL https://linkinghub.elsevier.com/retrieve/pii/S0009912018313778.

[70] Axel Schlitt, Christoph Bickel, Prathima Thumma, Stefan Blankenberg, Hans J. Rupprecht, Juergen Meyer, and Xian-Cheng Jiang. High Plasma Phospholipid Transfer Protein Levels as a Risk Factor for Coronary Artery Disease. Arteriosclerosis, Thrombosis, and Vascular Biology, 23(10):1857-1862, oct 2003. ISSN 10795642. doi: 10.1161/01.ATV.0000094433.98445.7F. URL https://www.ahajournals.org/doi/10.1161/01. ATV.0000094433.98445.7F

[71] Axel Schlitt, Stefan Blankenberg, Christoph Bickel, Karl J. Lackner, Gunnar H. Heine, Michael Buerke, Karl Werdan, Lars Maegdefessel, Uwe Raaz, Hans J. Rupprecht, Thomas Munzel, and Xian-Cheng Jiang. PLTP activity is a risk factor for subsequent cardiovascular events in CAD patients under statin therapy: the AtheroGene Study. Journal of Lipid Research, 50(4):723-729, apr 2009. ISSN 00222275. doi: 10.1194/jlr. M800414-JLR200. URL https://linkinghub.elsevier.com/retrieve/pii/S0022227520308701.

[72] Yu xing Zhao, Hui juan Zhu, Hui Pan, Xue mei Liu, Lin jie Wang, Hong bo Yang, Nai shi Li, Feng ying Gong, Wei Sun, and Yong Zeng. Comparative proteome analysis of epicardial and subcutaneous adipose tissues from patients with or without coronary artery disease. International Journal of Endocrinology, 2019(nil):1-11, 2019. doi: 10.1155/2019/6976712. URL https://doi.org/10.1155/2019/6976712.

[73] Qingyuan Zhao, Jingshu Wang, Wes Spiller, Jack Bowden, and Dylan S. Small. Two-sample instrumental variable analyses using heterogeneous samples. Statistical Science, 34(2):317-333, 2019. doi: 10.1214/18-sts692. URL https://doi.org/10.1214/18-sts692. 
[74] Verena Zuber, Johanna Maria Colijn, Caroline Klaver, and Stephen Burgess. Selecting causal risk factors from high-throughput experiments using multivariable Mendelian randomization. bioRxiv, page 396333, jan 2019. doi: 10.1101/396333. URL http://biorxiv.org/content/early/2019/05/20/396333.abstract.

[75] Sanna Kuusisto, Michael V Holmes, Pauli Ohukainen, Antti J Kangas, Mari Karsikas, Mika Tiainen, Markus Perola, Veikko Salomaa, Johannes Kettunen, and Mika Ala-Korpela. Direct Estimation of HDL-Mediated Cholesterol Efflux Capacity from Serum. Clinical Chemistry, 65(8):1042-1050, aug 2019. ISSN 0009-9147. doi: 10.1373/clinchem.2018.299222. URL https://doi.org/10.1373/clinchem.2018.299222. 


\section{Appendix 1 Lipid and lipoprotein traits}

Two published GWAS of the human lipidome [31, 32] measured lipoprotein subfractions and particle sizes using NMR spectroscopy. We investigated the 82 lipid and lipoprotein traits measured in these studies that are related to very-low-density lipoprotein (VLDL), LDL, and HDL subfractions and particle sizes. All the subfraction traits are named using three components separated by hyphen: the first indicates the size (XS, S, M, L, XL, XXL); the second indicates the category according to the lipoprotein density (VLDL, LDL, IDL, HDL); the third indicates the measurement ( $\mathrm{C}$ for total cholesterol, $\mathrm{CE}$ for cholesterol esters, FC for free cholesterol, $\mathrm{L}$ for total lipids, $\mathrm{P}$ for particle concentration, PL for phospholipids, TG for triglycerides). A full list of lipid and lipoprotein traits used in our study can be found in Appendix 1-Table 1 below.

Appendix 1-Table 1: All 82 traits included in this study and whether they are measured in the Kettunen and Davis GWAS (NA means not available).

\begin{tabular}{|c|c|c|c|}
\hline Trait & Description & Kettunen & Davis \\
\hline \multicolumn{4}{|c|}{ VLDL traits and total triglycerides } \\
\hline TG & Total triglycerides & & \\
\hline VLDL-D & VLDL diameter & & \\
\hline XS-VLDL-L & Total lipids in very small VLDL & & NA \\
\hline XS-VLDL-P & Concentration of very small VLDL particles & & \\
\hline XS-VLDL-PL & Phospholipids in very small VLDL & & \\
\hline XS-VLDL-TG & Triglycerides in very small VLDL & & \\
\hline S-VLDL-C & Total cholesterol in small VLDL & & \\
\hline S-VLDL-FC & Free cholesterol in small VLDL & & \\
\hline S-VLDL-L & Total lipids in small VLDL & & NA \\
\hline S-VLDL-P & Concentration of small VLDL particles & & \\
\hline S-VLDL-PL & Phospholipids in small VLDL & & \\
\hline S-VLDL-TG & Triglycerides in small VLDL & & \\
\hline M-VLDL-C & Total cholesterol in medium VLDL & & \\
\hline M-VLDL-CE & Cholesterol esters in medium VLDL & & \\
\hline M-VLDL-FC & Free cholesterol in medium VLDL & & \\
\hline M-VLDL-L & Total lipids in medium VLDL & & NA \\
\hline M-VLDL-P & Concentration of medium VLDL particles & & \\
\hline M-VLDL-PL & Phospholipids in medium VLDL & & \\
\hline M-VLDL-TG & Triglycerides in medium VLDL & & \\
\hline L-VLDL-C & Total cholesterol in large VLDL & & \\
\hline L-VLDL-CE & Cholesterol esters in large VLDL & & \\
\hline L-VLDL-FC & Free cholesterol in large VLDL & & \\
\hline L-VLDL-L & Total lipids in large VLDL & & NA \\
\hline L-VLDL-P & Concentration of large VLDL particles & & \\
\hline L-VLDL-PL & Phospholipids in large VLDL & & \\
\hline L-VLDL-TG & Triglycerides in large VLDL & & \\
\hline XL-VLDL-L & Total lipids in very large VLDL & & NA \\
\hline XL-VLDL-P & Concentration of very large VLDL particles & & \\
\hline XL-VLDL-PL & Phospholipids in very large VLDL & & \\
\hline XL-VLDL-TG & Triglycerides in very large VLDL & & \\
\hline XXL-VLDL-L & Total lipids in chylomicrons and extremely very large VLDL & & NA \\
\hline XXL-VLDL-P & Concentration of chylomicrons and extremely very large VLDL particles & & \\
\hline XXL-VLDL-PL & Phospholipids in chylomicrons and extremely very large & & \\
\hline XXL-VLDL-TG & Triglycerides in chylomicrons and extremely very large & & \\
\hline \multicolumn{4}{|c|}{ LDL and IDL traits } \\
\hline LDL-C & Total cholesterol in LDL & & \\
\hline ApoB & Apolipoprotein B & & \\
\hline LDL-D & LDL diameter & & \\
\hline S-LDL-C & Total cholesterol in small LDL & & \\
\hline S-LDL-L & Total lipids in small LDL & & NA \\
\hline
\end{tabular}




\begin{tabular}{|c|c|c|}
\hline S-LDL-P & Phospholipids in small LDL & \\
\hline M-LDL-C & Total cholesterol in medium LDL & \\
\hline M-LDL-CE & Cholesterol esters in medium LDL & \\
\hline M-LDL-L & Total lipids in medium LDL & NA \\
\hline M-LDL-P & Concentration of medium LDL particles & \\
\hline M-LDL-PL & Phospholipids in medium LDL & \\
\hline L-LDL-C & Total cholesterol in large LDL & \\
\hline L-LDL-CE & Cholesterol esters in large LDL & \\
\hline L-LDL-FC & Free cholesterol in large LDL & \\
\hline L-LDL-L & Total lipids in large LDL & NA \\
\hline L-LDL-P & Concentration of large LDL particles & \\
\hline L-LDL-PL & Phospholipids in large LDL & \\
\hline IDL-C & Total cholesterol in IDL & \\
\hline IDL-FC & Free cholesterol in IDL & \\
\hline IDL-L & Total lipids in IDL & NA \\
\hline IDL-P & Concentration of IDL particles & \\
\hline IDL-PL & Phospholipids in IDL & \\
\hline IDL-TG & Triglycerides in IDL & \\
\hline \multicolumn{3}{|l|}{ HDL traits } \\
\hline HDL-C & Total cholesterol in HDL & \\
\hline ApoA1 & Apolipoprotein A1 & \\
\hline HDL-D & HDL diameter & \\
\hline S-HDL-L & Total lipids in small HDL & NA \\
\hline S-HDL-P & Concentration of small HDL particles & \\
\hline S-HDL-TG & Triglycerides in small HDL & \\
\hline M-HDL-C & Total cholesterol in medium HDL & \\
\hline M-HDL-CE & Cholesterol esters in medium HDL & \\
\hline M-HDL-FC & Free cholesterol in medium HDL & \\
\hline M-HDL-L & Total lipids in medium HDL & NA \\
\hline M-HDL-P & Concentration of medium HDL particles & \\
\hline M-HDL-PL & Phospholipids in medium HDL & \\
\hline L-HDL-C & Total cholesterol in large HDL & \\
\hline L-HDL-CE & Cholesterol esters in large HDL & \\
\hline L-HDL-FC & Free cholesterol in large HDL & \\
\hline L-HDL-L & Total lipids in large HDL & NA \\
\hline L-HDL-P & Concentration of large HDL particles & \\
\hline L-HDL-PL & Phospholipids in large HDL & \\
\hline XL-HDL-C & Total cholesterol in very large HDL & \\
\hline XL-HDL-CE & Cholesterol esters in very large HDL & \\
\hline XL-HDL-FC & Free cholesterol in very large HDL & \\
\hline XL-HDL-L & Total lipids in very large HDL & NA \\
\hline XL-HDL-P & Concentration of very large HDL particles & \\
\hline XL-HDL-PL & Phospholipids in very large HDL & \\
\hline XL-HDL-TG & Triglycerides in very large HDL & \\
\hline
\end{tabular}


bioRxiv preprint doi: https://doi org/10.1101/691089; this version posted February 6, 2021. The copyright holder for this preprint (which was not certified by peer review) is the author/funder, who has granted bioRxiv a license to display the preprint in perpetuity. It is made available under aCC-BY-NC-ND 4.0 International license.

\section{Appendix 2 Genetic Correlations}

We estimated the genetic correlation between lipoprotein subfractions, particle sizes, and traditional lipid risk factors using the LD score regression [37. Appendix 2-Figure 1 to 3 show the estimated genetic correlation matrix between selected traits using different datasets. Below the figures, Appendix 2-Table1 1 shows the estimated genetic correlations of the lipoprotein subbfractions with the traditional lipid risk factors using the Davis GWAS. The results in Appendix 2-Table 1 were then used to screen the traits as described in Materials and Methods.

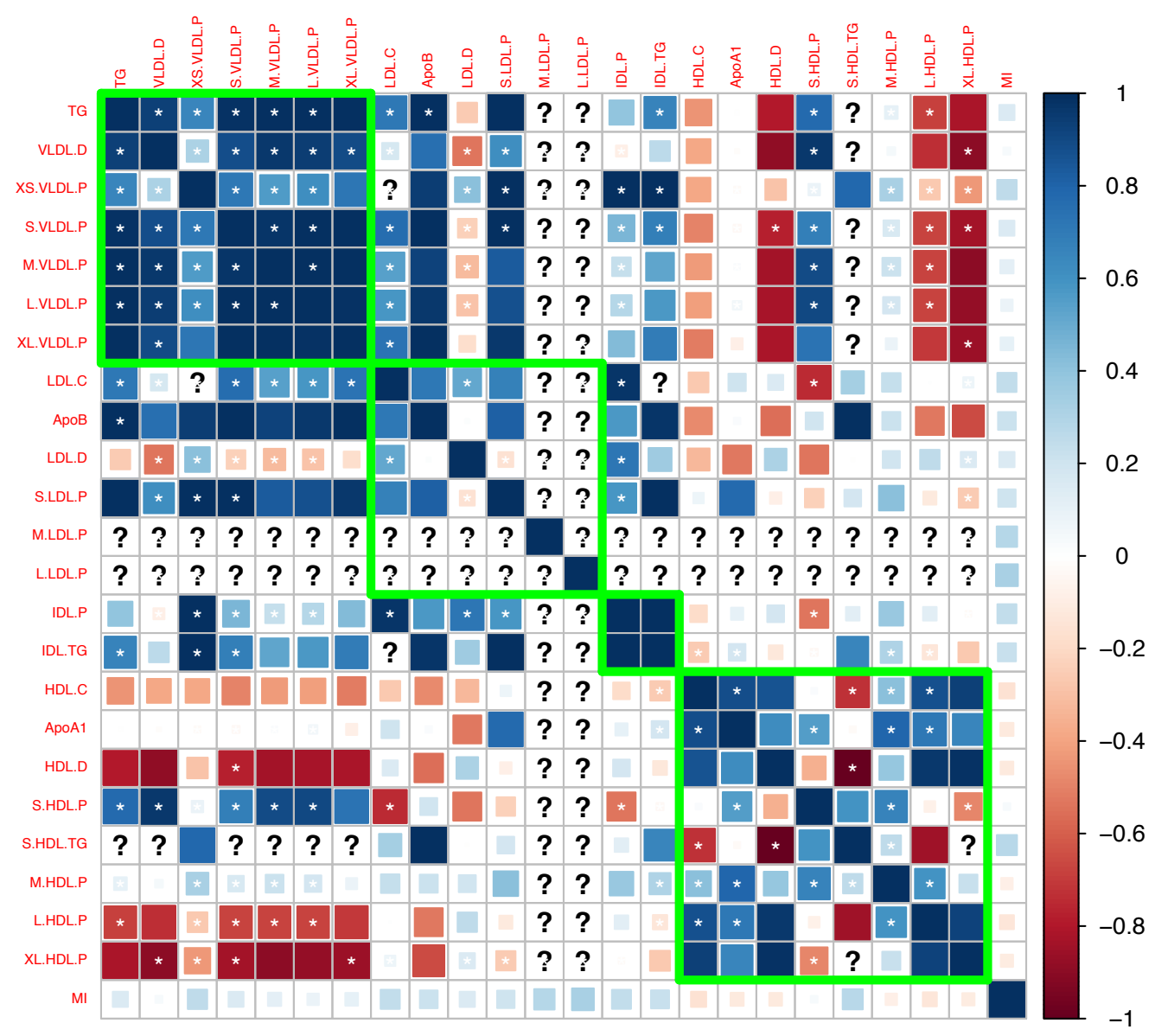

Appendix 2-Figure 1: Genetic correlations computed using the Davis et al. (2017) GWAS summary dataset. 
bioRxiv preprint doi: https://doi.org/10.1101/691089; this version posted February 6, 2021. The copyright holder for this preprint (which was not certified by peer review) is the author/funder, who has granted bioRxiv a license to display the preprint in perpetuity. It is made available under aCC-BY-NC-ND 4.0 International license.

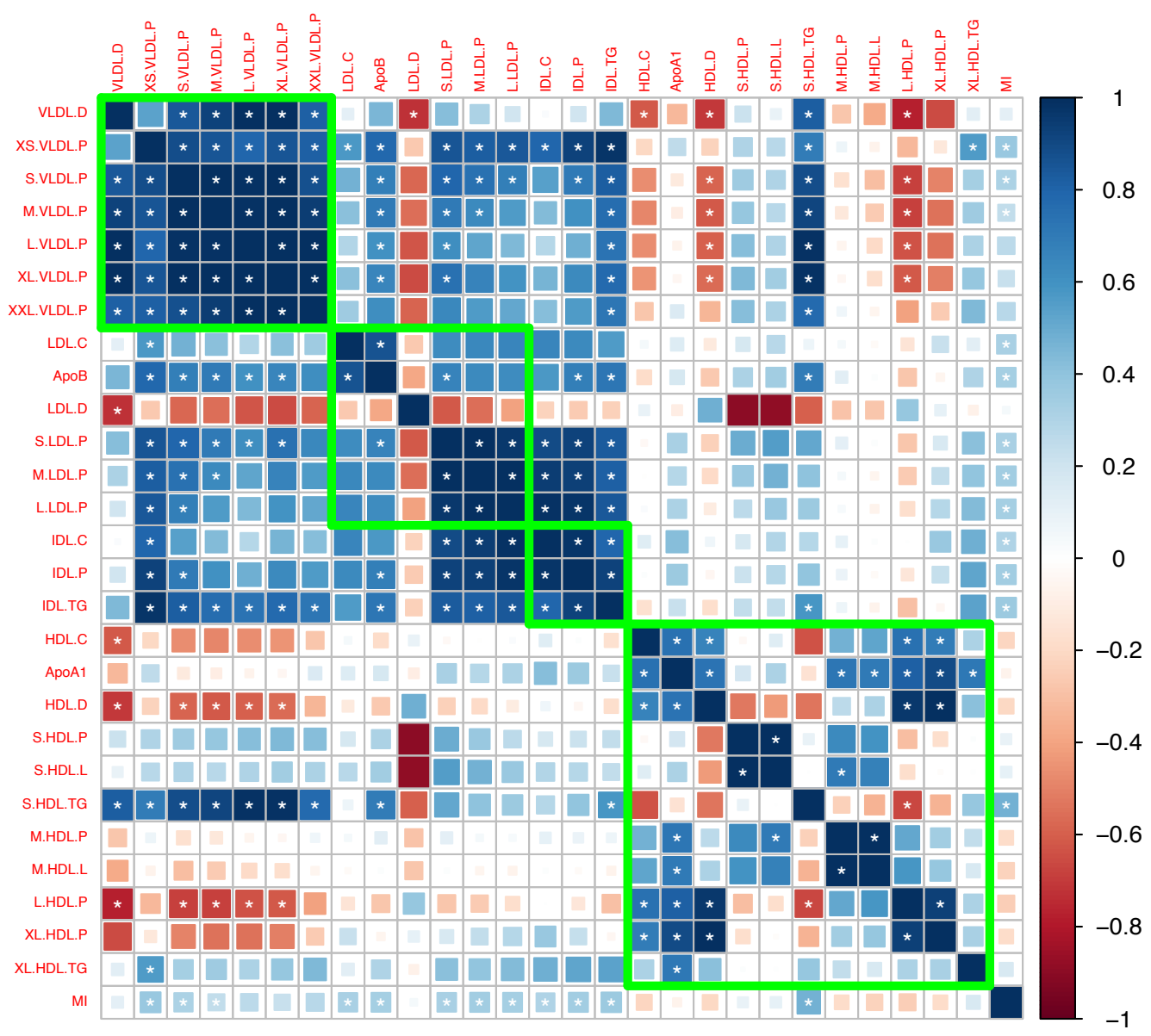

Appendix 2-Figure 2: Genetic correlations computed using the Kettunen et al. (2016) GWAS summary dataset. 
bioRxiv preprint doi: https://doi.org/10.1101/691089; this version posted February 6, 2021. The copyright holder for this preprint (which was not certified by peer review) is the author/funder, who has granted bioRxiv a license to display the preprint in perpetuity. It is made available under aCC-BY-NC-ND 4.0 International license.

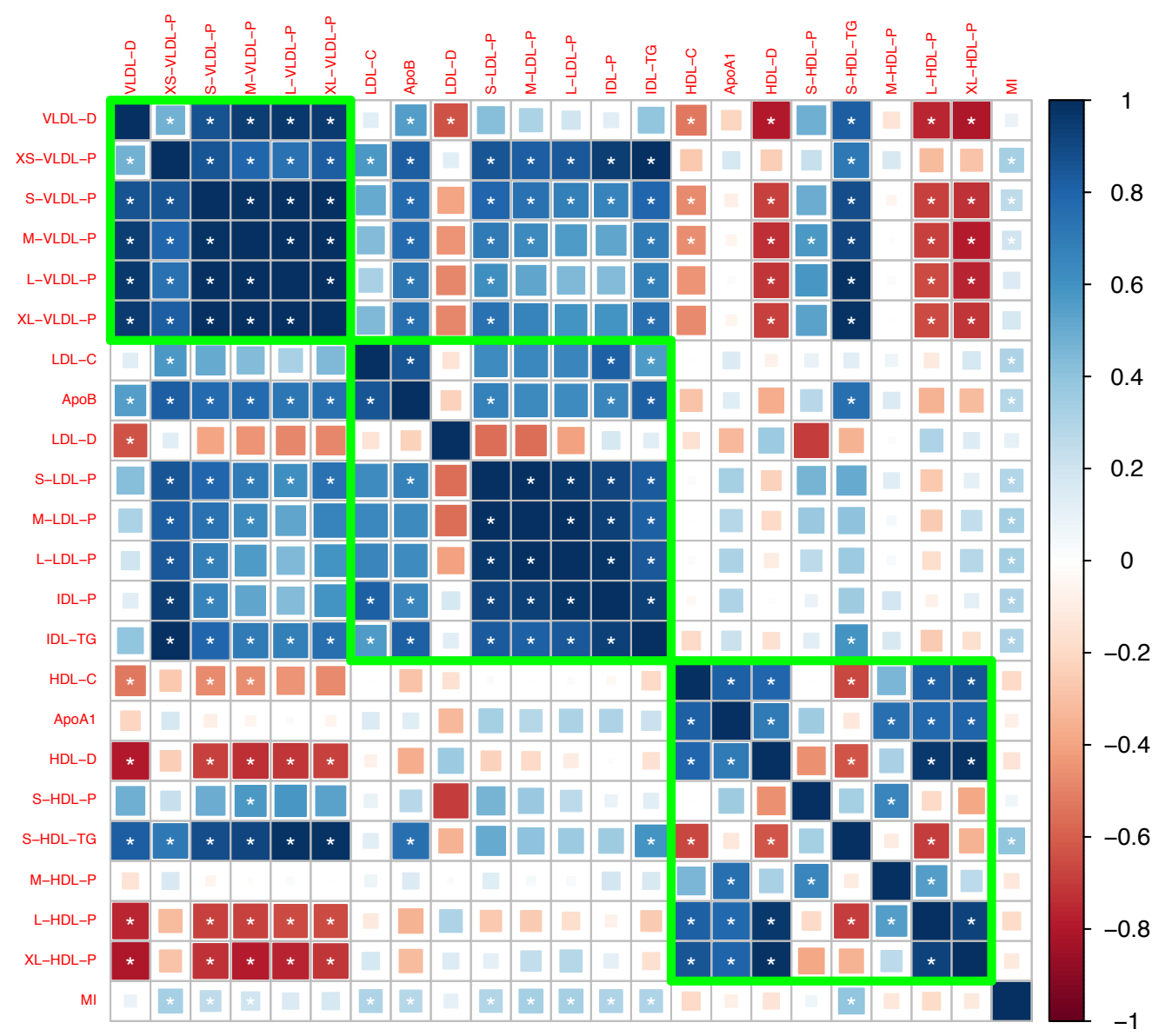

Appendix 2-Figure 3: Genetic correlations computed by meta-analyzing the results in Appendix 2-Figure 1 and 2 
Appendix 2-Table 1: Estimated genetic correlation (standard error) of the lipoprotein subfractions with the traditional lipid risk factors using the Davis GWAS. Bolded estimates are above 0.8 and the corresponding traits were removed in phenotypic screening.

\begin{tabular}{|c|c|c|c|c|c|}
\hline Trait & ApoA1 & ApoB & HDL-C & LDL-C & TG \\
\hline S-HDL-L & $0.31(0.28)$ & $0.34(0.25)$ & $0.13(0.26)$ & $0.27(0.3)$ & $0.2(0.22)$ \\
\hline S-HDL-P & $0.36(0.24)$ & $0.27(0.22)$ & $-0.01(0.22)$ & $0.1(0.31)$ & $0.48(0.17)$ \\
\hline S-HDL-TG & $-0.13(0.25)$ & $0.77(0.13)$ & $-0.66(0.15)$ & $0.13(0.28)$ & $1.03(0.07)$ \\
\hline M-HDL-C & $0.65(0.14)$ & $-0.18(0.2)$ & $0.81(0.09)$ & $-0.09(0.25)$ & $-0.34(0.17)$ \\
\hline M-HDL-CE & $0.68(0.14)$ & $-0.23(0.21)$ & $0.57(0.12)$ & $-0.24(0.24)$ & $-0.32(0.18)$ \\
\hline M-HDL-FC & $0.67(0.12)$ & $-0.08(0.21)$ & $0.83(0.08)$ & $0.04(0.24)$ & $-0.28(0.18)$ \\
\hline M-HDL-L & $0.71(0.15)$ & $0.02(0.27)$ & $0.52(0.17)$ & $-0.03(0.29)$ & $-0.19(0.25)$ \\
\hline M-HDL-P & $0.75(0.12)$ & $0.15(0.23)$ & $0.46(0.14)$ & $0.08(0.26)$ & $0(0.19)$ \\
\hline M-HDL-PL & $0.69(0.13)$ & $0.04(0.22)$ & $0.65(0.11)$ & $0.02(0.25)$ & $-0.04(0.19)$ \\
\hline L-HDL-C & $0.76(0.11)$ & $-0.42(0.13)$ & $0.95(0.02)$ & $-0.1(0.18)$ & $-0.62(0.09)$ \\
\hline L-HDL-CE & $0.82(0.1)$ & $-0.4(0.12)$ & $0.93(0.04)$ & $-0.16(0.17)$ & $-0.62(0.09)$ \\
\hline L-HDL-FC & $0.66(0.12)$ & $-0.46(0.13)$ & $0.92(0.03)$ & $-0.13(0.18)$ & $-0.7(0.08)$ \\
\hline L-HDL-L & $0.81(0.11)$ & $-0.29(0.15)$ & $0.74(0.07)$ & $-0.15(0.18)$ & $-0.56(0.12)$ \\
\hline L-HDL-P & $0.79(0.09)$ & $-0.35(0.13)$ & $0.82(0.05)$ & $-0.12(0.16)$ & $-0.61(0.09)$ \\
\hline L-HDL-PL & $0.77(0.09)$ & $-0.34(0.13)$ & $0.79(0.05)$ & $-0.12(0.17)$ & $-0.61(0.09)$ \\
\hline XL-HDL-C & $0.75(0.16)$ & $-0.25(0.19)$ & $0.9(0.1)$ & $0.4(0.27)$ & $-0.63(0.13)$ \\
\hline XL-HDL-CE & $0.82(0.16)$ & $-0.17(0.19)$ & $0.82(0.09)$ & $0.41(0.27)$ & $-0.54(0.12)$ \\
\hline XL-HDL-FC & $0.72(0.14)$ & $-0.37(0.18)$ & $0.94(0.08)$ & $0.17(0.23)$ & $-0.71(0.11)$ \\
\hline XL-HDL-L & $0.93(0.16)$ & $-0.08(0.25)$ & $0.68(0.14)$ & $0.1(0.27)$ & $-0.35(0.2)$ \\
\hline XL-HDL-P & $0.81(0.13)$ & $-0.32(0.16)$ & $0.86(0.08)$ & $0.17(0.21)$ & $-0.69(0.11)$ \\
\hline XL-HDL-PL & $0.76(0.12)$ & $-0.41(0.15)$ & $0.83(0.07)$ & $-0.09(0.18)$ & $-0.7(0.09)$ \\
\hline XL-HDL-TG & $0.72(0.13)$ & $0.49(0.17)$ & $0.33(0.13)$ & $0.13(0.26)$ & $0.3(0.15)$ \\
\hline HDL-D & $0.7(0.11)$ & $-0.36(0.13)$ & $0.8(0.06)$ & $-0.08(0.17)$ & $-0.64(0.09)$ \\
\hline IDL-C & $0.38(0.21)$ & $0.58(0.19)$ & $0.07(0.19)$ & $0.8(0.14)$ & $0.39(0.17)$ \\
\hline IDL-FC & $0.23(0.2)$ & $0.78(0.12)$ & $-0.05(0.17)$ & $0.61(0.19)$ & $0.44(0.15)$ \\
\hline IDL-L & $0.38(0.23)$ & $0.65(0.18)$ & $0.05(0.2)$ & $0.64(0.2)$ & $0.47(0.17)$ \\
\hline IDL-P & $0.31(0.2)$ & $0.66(0.14)$ & $-0.04(0.17)$ & $0.82(0.13)$ & $0.49(0.14)$ \\
\hline IDL-PL & $0.25(0.23)$ & $0.83(0.1)$ & $-0.12(0.19)$ & $0.7(0.19)$ & $0.64(0.15)$ \\
\hline IDL-TG & $0.22(0.18)$ & $0.82(0.08)$ & $-0.2(0.13)$ & $0.56(0.15)$ & $0.67(0.08)$ \\
\hline S-LDL-C & $0.11(0.28)$ & $0.66(0.18)$ & $-0.16(0.22)$ & $0.44(0.34)$ & $0.58(0.14)$ \\
\hline S-LDL-L & $0.26(0.23)$ & $0.66(0.17)$ & $-0.06(0.21)$ & $0.62(0.21)$ & $0.58(0.13)$ \\
\hline S-LDL-P & $0.34(0.2)$ & $0.68(0.15)$ & $-0.02(0.19)$ & $0.63(0.18)$ & $0.58(0.13)$ \\
\hline M-LDL-C & $0.15(0.26)$ & $0.63(0.18)$ & $0.22(0.22)$ & $0.87(0.08)$ & $0.13(0.23)$ \\
\hline M-LDL-CE & $0.3(0.23)$ & $0.61(0.2)$ & $0.05(0.21)$ & $0.65(0.2)$ & $0.45(0.16)$ \\
\hline M-LDL-L & $0.29(0.22)$ & $0.63(0.18)$ & $0.01(0.21)$ & $0.66(0.19)$ & $0.5(0.15)$ \\
\hline M-LDL-P & $0.29(0.23)$ & $0.63(0.18)$ & $-0.01(0.21)$ & $0.65(0.21)$ & $0.51(0.15)$ \\
\hline M-LDL-PL & $0.2(0.24)$ & $0.69(0.16)$ & $0.11(0.2)$ & $0.89(0.06)$ & $0.18(0.22)$ \\
\hline L-LDL-C & $0.25(0.24)$ & $0.58(0.21)$ & $0.25(0.22)$ & $0.68(0.19)$ & $0.23(0.21)$ \\
\hline L-LDL-CE & $0.3(0.23)$ & $0.58(0.22)$ & $0.05(0.21)$ & $0.65(0.21)$ & $0.41(0.17)$ \\
\hline L-LDL-FC & $0.31(0.24)$ & $0.57(0.22)$ & $0.33(0.23)$ & $0.7(0.18)$ & $0.13(0.23)$ \\
\hline L-LDL-L & $0.31(0.23)$ & $0.61(0.2)$ & $0.04(0.21)$ & $0.65(0.21)$ & $0.44(0.17)$ \\
\hline L-LDL-P & $0.31(0.23)$ & $0.63(0.19)$ & $0.02(0.21)$ & $0.65(0.21)$ & $0.47(0.16)$ \\
\hline
\end{tabular}




\begin{tabular}{|c|c|c|c|c|c|}
\hline L-PL & $0.27(0.25)$ & $0.61(0.2)$ & $0.24(0.22)$ & $(0.2)$ & 2) \\
\hline LDL-D & $-0.33(0.25)$ & $-0.22(0.23)$ & $-0.15(0.21)$ & $-0.15(0.29)$ & $-0.37(0.16)$ \\
\hline XS-VLDL-L & $0.25(0.23)$ & $0.8(0.08)$ & $-0.2(0.17)$ & $0.61(0.14)$ & $0.73(0.09)$ \\
\hline XS-VLDL-P & $0.17(0.18)$ & $0.83(0.07)$ & $-0.26(0.13)$ & $0.57(0.13)$ & $0.71(0.07)$ \\
\hline XS-VLDL-PL & $21(0.19)$ & $.78(0.09)$ & $-0.15(0.15)$ & $0.74(0.14)$ & $0.57(0.11)$ \\
\hline XS-VLDL-TG & $.06(0.18)$ & $0.83(0$ & $-0.37(C$ & 0.56 & 0.85 \\
\hline S-VLDL-FC & $-0.08(0.2)$ & $0.94(0.05)$ & $-0.49(0.12)$ & $0.59(0.12)$ & $0.92(0.03)$ \\
\hline S-VLDL-L & $-0.12(0.24)$ & $0.7(0.08)$ & $-0.46(0.15)$ & $0.5(0.14)$ & $0.8(0.05)$ \\
\hline -VLDL-P & $-0.09(0.19)$ & $0.78(0.07)$ & $-0.48(0$ & $0.5(0.14)$ & $0.95(0.02)$ \\
\hline S-VLDL-PL & $-0.03(0.2)$ & $0.82(0.08)$ & $-0.43(0.12)$ & $0.44(0.17)$ & $0.92(0.03)$ \\
\hline S-VLDL-TG & $-0.1(0.2)$ & $0.9(0.08)$ & $-0.49(0.11)$ & $0.49(0.15)$ & $0.98(0.01)$ \\
\hline S-VLDL-C & 0.01 & $0.9(0.06)$ & $-0.39(0.13)$ & 0.61 & $0.89(0.05)$ \\
\hline M-VLDL-C & $-0.01(0.2)$ & $0.8(0.09)$ & $-0.47(0.12)$ & 0.41 & $0.95(0.02)$ \\
\hline M-VLDL-CE & $0.01(0.19)$ & $0.78(0.08)$ & -0.43 & 0.5( & $0.9(0.03)$ \\
\hline$-\mathrm{FC}$ & $0(0$. & $0.83(0.09)$ & -0.48 & & 0.97 \\
\hline M-VLDL-L & $-0.1(0.24)$ & $0.66(0.11)$ & $-0.48(0.15)$ & 0.4 & $0.8(0.05)$ \\
\hline M-VLDL-P & $-0.06(0.19)$ & $0.78(0.1)$ & $-0.46(0.12)$ & $0.43(0.16)$ & $0.98(0.02)$ \\
\hline M-VLDL-PL & $0.03(0.21)$ & $0.85(0.09)$ & $-0.48(0.12)$ & $0.4(0.18)$ & $0.98(0.01)$ \\
\hline M-VLDL-TG & $-0.02(0.21)$ & $0.82(0.11)$ & $-0.5(0.13)$ & 0.33 & $0.98(0.02)$ \\
\hline L-VLDL-C & -0.05 & & & & 2) \\
\hline L-VLDL-CE & $0(0.19)$ & $0.78(0.12)$ & -0.44 & 0.43 & $0.03)$ \\
\hline L-VLDL-FC & $-0.03(0.2)$ & $0.84(0.12)$ & $-0.53(0.13)$ & $0.36(0.19)$ & 1( \\
\hline L-VLDL-L & $-0.06(0.24)$ & $0.66(0.14)$ & $-0.47(0.16)$ & $0.36(0.2)$ & $0.86(0.05)$ \\
\hline L-VLDL-P & $-0.02(0.21)$ & $0.72(0.12)$ & $-0.44(0.13)$ & $0.33(0.18)$ & $0.98(0.02)$ \\
\hline L-VLDL-PL & $0.01(0.21)$ & $0.86(0.12)$ & $-0.53(0.13)$ & $0.3(0.2)$ & $1.04(0.03)$ \\
\hline L-VLDL-TG & $-0.06(0.21)$ & $0.78(0.12)$ & $-0.54(0.13)$ & $0.26(0.19)$ & $1(0.02)$ \\
\hline XL-VLDL-L & $-0.08(0.24)$ & $0.7(0.15)$ & $-0.52(0.16)$ & $0.43(0.2)$ & $0.85(0.05)$ \\
\hline XL-VLDL-P & $-0.06(0.2)$ & $0.76(0.12)$ & $-0.48(0.13)$ & $0.44(0.18)$ & $0.95(0.03)$ \\
\hline XL-VLDL-PL & $-0.09(0.23)$ & $0.82(0.13)$ & $-0.62(0.15)$ & $0.32(0.21)$ & $1.06(0.04)$ \\
\hline XL-VLDL-TG & $-0.14(0.21)$ & $0.86(0.13)$ & $-0.65(0.13)$ & $0.34(0.19)$ & $1.03(0.04)$ \\
\hline XXL-VLDL-L & $-0.07(0.25)$ & $0.65(0.16)$ & $-0.5(0.17)$ & $0.38(0.22)$ & $0.83(0.06)$ \\
\hline XXL-VLDL-P & $0.17(0.2)$ & $0.72(0.15)$ & $-0.3(0.15)$ & $0.39(0.21)$ & $0.86(0.07)$ \\
\hline XXL-VLDL-PL & $-0.3(0.24)$ & $0.66(0.17)$ & $-0.8(0.16)$ & $0.22(0.21)$ & $1.06(0.06)$ \\
\hline XXL-VLDL-TG & $-0.21(0.25)$ & $0.64(0.16)$ & $-0.7(0.15)$ & $0.22(0.22)$ & $1.08(0.05)$ \\
\hline VLDL-D & $-0.22(0.2)$ & $0.55(0.14)$ & $-0.53(0.12)$ & $0.12(0.19)$ & $0.86(0.04)$ \\
\hline
\end{tabular}




\section{Appendix 3 Mendelian randomization}

We implemented several Mendelian randomization (MR) designs and statistical methods to estimate the causal effect of lipoprotein subfractions and particles sizes on coronary artery disease. In general, we adopted the threesample summary data MR design described in Zhao et al. [38, Wang et al. [44] and we swapped the roles of the GWAS datasets whenever permitted by the statistical methods. More specifically, the statistical methods we used for univariable MR (RAPS, IVW, weighted median) require that the GWAS datasets for obtaining instruments, SNP effects on the exposure, and SNP effects on the outcome must have no overlapping sample. The multivariable MR method we used (GRAPPLE) allows the exposure and outcome GWAS to be dependent and estimates the proportion of overlapping sample. However, GRAPPLE still requires that the selection GWAS uses an nonoverlapping sample.

The MR designs we implemented in this study are summarized in Appendix 3-Table 1 We considered two ways of instrument selection for univariable MR. In "traditional selection", the traditional lipid traits were used to select the instruments for the corresponding subfraction traits. That is, HDL-C was used to select SNPs for HDL subfractions and particle size, LDL-C for IDL and LDL subfractions and particle size, and TG for VLDL subfractions and particle size. This tends to select more instruments because the GWAS for traditional lipid traits had a larger sample size. In "subfraction selection", the instrumental SNPs were selected for each lipoprotein subfraction and particle size using the same or closest trait in the selection GWAS. For example, if the exposure under investigation is S-HDL-L but it is not measured in the Davis GWAS (if it is used for selection), S-HDL-P is used instead for instrument selection.

For multivariable MR, we considered two models with different sets of exposures: TG, LDL-C, HDL-C, and the subfraction/particle size under investigation; TG, ApoB, ApoA1, and the subfraction/particle size under investigation. SNPs were selected as potential instruments if they were associated (p-value $\leq 10^{-4}$ ) with at least one of the four exposures. LD clumping was then used to obtain independent instruments, as described in Materials and Methods.

We briefly comment on the statistical methods used in univariable MR. All the three methods we used-RAPS, IVW, weighted median - require that the exposure GWAS and outcome GWAS have non-overlapping samples. RAPS and weighted median can provide consistent estimate of the causal effect even when some of the genetic variants are not valid instruments, provided that the direct effects of the genetic variants are independent of the strength of their associations with the exposure. The last condition is called the Instrument Strength Independent of Direct Effect (InSIDE) assumption in the MR literature [50]. RAPS is also robust to idiosyncratically large direct effect 43. Because IVW and weighted median can be severely biased by weak instruments [43, we only used them with the set of SNPs that have genome-wide significant association (p-value $\leq 5 \times 10^{-8}$ ) with the exposure. In comparison, RAPS does not suffer from weak instrument bias and we used it with all the SNPs obtained by LD clumping without any p-value threshold.

Below, Appendix 3-Figure 1 shows the MR results for the 27 lipoprotein measurements selected in phenotypic screening. Estimates that are statistically significant at a false discovery rate of 0.05 are shown in Figure 2 of the main paper. Appendix 3-Table 2 shows the estimated effect of all the lipoprotein subfractions and particle sizes on myocardial infarction or coronary artery disease in various MR designs. Full results of the multivariable MR analyses, including the estimated effects of the traditional lipid risk factors, can be found in Appendix 3-Table 5 and 6. The results of the univariable MR analyses using IVW and weighted median estimators can be found in Appendix 3-Table 3 and 4 


\begin{tabular}{|c|c|c|c|c|}
\hline MR Design & Selection & Exposure & Outcome & Reported in \\
\hline \multirow{4}{*}{$\begin{array}{l}\text { Univariable } \\
\text { (traditional selection) }\end{array}$} & GERA & Davis & CARDIoGRAMplusC4D & Appendix 3-Table 2 to 4 \\
\hline & GERA & Davis & UK Biobank & Appendix 3 -Table 2 to \\
\hline & GERA & Kettunen & UK Biobank & Appendix 3-Table 2 to 4 \\
\hline & GLGC & Davis & UK Biobank & Appendix 3-Table 2 to 4 \\
\hline \multirow{2}{*}{$\begin{array}{l}\text { Univariable } \\
\text { (subfraction selection) }\end{array}$} & Davis & Kettunen & UK Biobank & $\begin{array}{c}\text { Figure 2; } \\
\text { Appendix 3-Figure } 1 \text { and appendix 3-table } 2 \text { to } 4\end{array}$ \\
\hline & Kettunen & Davis & UK Biobank & Appendix 3-Figure 1 and appendix 3 -table 2 to 4 \\
\hline Multivariable & Davis, GERA & Kettunen, GLGC & $\begin{array}{c}\text { CARDIoGRAMplusC4D } \\
+ \text { UK Biobank }\end{array}$ & $\begin{array}{l}\text { Figure 2, Table 2; } \\
\text { Appendix 3-Figure } 1 \text { and appendix 3-table } 2 \text { to } 4\end{array}$ \\
\hline
\end{tabular}

Appendix 3-Table 1: Three-sample Mendelian randomization designs. 


\section{Appendix 3.1 Pooled results}
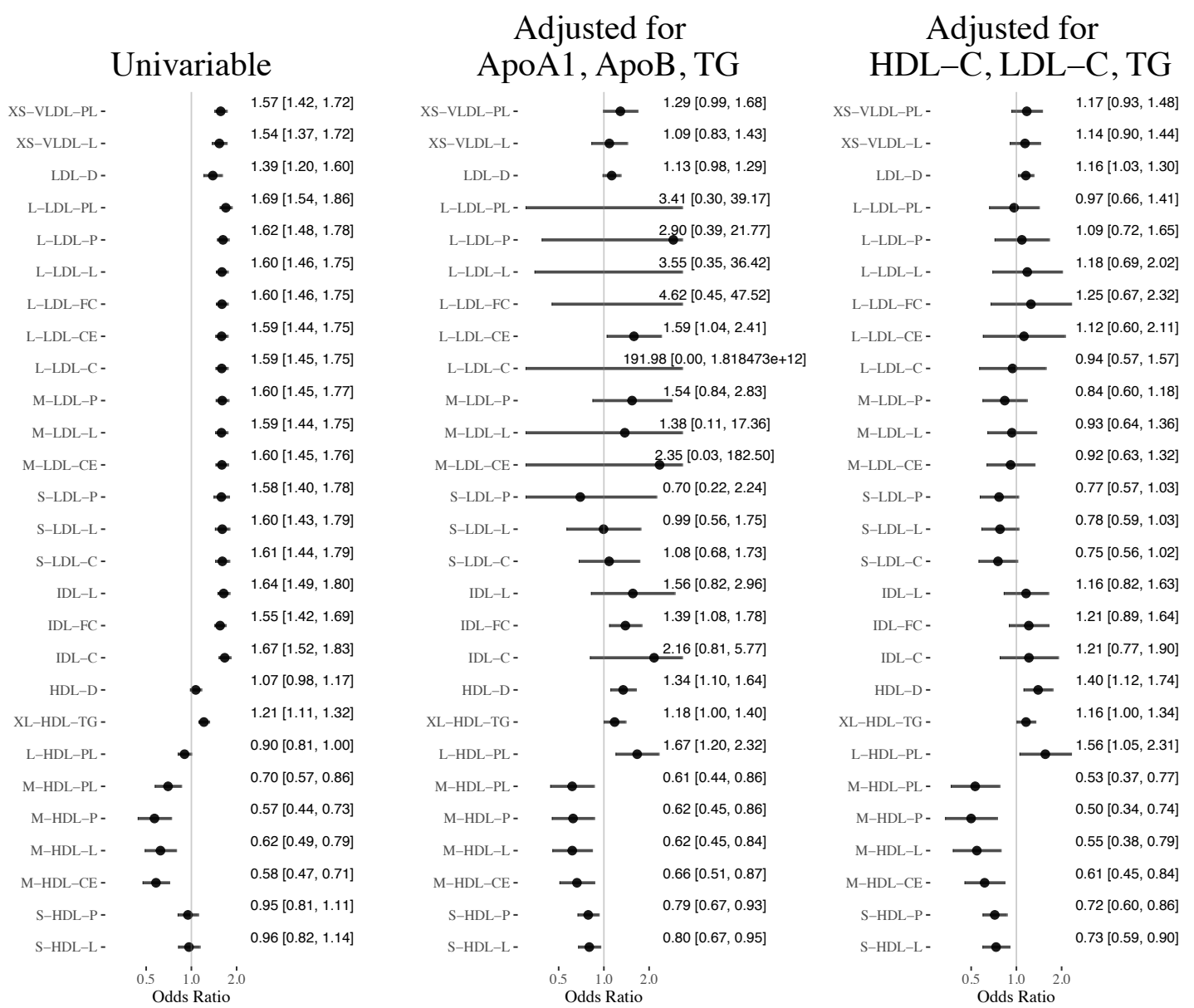

Appendix 3-Figure 1: Mendelian randomization results for the 27 lipoprotein measurements selected in phenotypic screening. 
In the tables below, Red indicates $p$-value is significant (at level 0.05) after Bonferroni correction for all the results in the corresponding table and blue indicates $p$-value $\leq 0.05$

Appendix 3-Table 2: Mendelian randomization results using all selected SNPs (univariable MR using RAPS and multivariable MR using GRAPPLE).

\begin{tabular}{|c|c|c|c|c|c|c|c|c|c|}
\hline \multirow{5}{*}{$\begin{array}{l}\text { Screening } \\
\text { Exposure } \\
\text { Outcome } \\
\text { Adjusted }\end{array}$} & \multicolumn{8}{|c|}{ Method: RAPS/GRAPPLE + All SNPs } & 离言 \\
\hline & GERA & GERA & GERA & GLGC & Davis & Kettunen & GERA + Davis & GERA + Davis & \\
\hline & Davis & Davis & Kettunen & Davis & Kettunen & Davis & GLGC + Kettunen & GLGC + Kettunen & \\
\hline & CAD & UKB & UKB & UKB & UKB & UKB & $\mathrm{CAD}+\mathrm{UKB}$ & $\mathrm{CAD}+\mathrm{UKB}$ & \\
\hline & & & & & & & HDL-C + LDL-C + TG & ApoA1 + ApoB + TG & \\
\hline \multicolumn{9}{|l|}{ VLDL traits } & \\
\hline TG & $.258(.053)$ & $.296(.075)$ & NA & $.262(.06)$ & NA & $.289(.068)$ & NA & NA & \\
\hline VLDL-D & $-.099(.049)$ & $.028(.074)$ & $.072(.073)$ & $.116(.065)$ & $-.163(.067)$ & $-.204(.071)$ & $-.588(.094)$ & $-.32(.112)$ & \\
\hline XS-VLDL-L & NA & $\mathrm{NA}$ & $.368(.064)$ & NA & $.429(.059)$ & $\mathrm{NA}$ & $.132(.119)$ & $.084(.141)$ & \\
\hline XS-VLDL-P & $.17(.031)$ & $.26(.048)$ & $.367(.065)$ & $.248(.047)$ & $.429(.06)$ & $.338(.056)$ & $.118(.125)$ & $.061(.158)$ & \\
\hline XS-VLDL-PL & $.191(.034)$ & $.284(.055)$ & $.386(.069)$ & $.278(.052)$ & $.449(.049)$ & $.435(.049)$ & $.159(.12)$ & $.253(.135)$ & \\
\hline XS-VLDL-TG & $.201(.034)$ & $.3(.053)$ & $.388(.068)$ & $.283(.046)$ & $.372(.063)$ & $.326(.055)$ & $-.157(.187)$ & $-.248(.15)$ & \\
\hline S-VLDL-C & $.294(.06)$ & $.343(.076)$ & NA & $.322(.063)$ & NA & $.424(.094)$ & $-1.035(.323)$ & $-1.265(.568)$ & \\
\hline S-VLDL-FC & $.243(.051)$ & $.303(.068)$ & $.389(.079)$ & $.286(.056)$ & $.489(.071)$ & $.416(.074)$ & $-1.027(.337)$ & $-.489(.213)$ & \\
\hline S-VLDL-L & NA & NA & $.356(.075)$ & NA & $.376(.072)$ & $\mathrm{NA}$ & $-.898(.28)$ & $-1.629(.586)$ & \\
\hline S-VLDL-P & $.226(.047)$ & $.288(.068)$ & $.343(.074)$ & $.261(.054)$ & $.359(.069)$ & $.271(.094)$ & $-1.245(.463)$ & $-1.644(.606)$ & \\
\hline S-VLDL-PL & $.228(.047)$ & $.294(.067)$ & $.372(.074)$ & $.273(.054)$ & $.365(.066)$ & $.336(.063)$ & $-.613(.182)$ & $-1.213(.478)$ & \\
\hline S-VLDL-TG & $.223(.049)$ & $.283(.071)$ & $.323(.073)$ & $.25(.055)$ & $.327(.071)$ & $.275(.067)$ & $\mathrm{NaN}$ & $-.301(.108)$ & \\
\hline M-VLDL-C & $.253(.053)$ & $.304(.078)$ & $.327(.074)$ & $.276(.06)$ & $.368(.07)$ & $.312(.079)$ & $-1.433(.451)$ & $-.373(.118)$ & $\frac{8}{2}$ \\
\hline M-VLDL-CE & $.248(.051)$ & $.309(.074)$ & $.344(.077)$ & $.285(.058)$ & $.369(.073)$ & $.295(.069)$ & $-1.035(.293)$ & $-.995(.338)$ & \\
\hline M-VLDL-FC & $.245(.058)$ & $.283(.082)$ & $.31(.076)$ & $.259(.063)$ & $.341(.069)$ & $.341(.068)$ & $-1.412(.444)$ & $-.799(.311)$ & \\
\hline M-VLDL-L & NA & NA & $.311(.079)$ & NA & $.358(.078)$ & $\mathrm{NA}$ & $-1.878(.75)$ & $-.298(.098)$ & \\
\hline M-VLDL-P & $.25(.062)$ & $.282(.083)$ & $.305(.081)$ & $.247(.065)$ & $.293(.089)$ & $.269(.065)$ & $-1.974(.745)$ & $-.312(.096)$ & 을 \\
\hline M-VLDL-PL & $.248(.056)$ & $.295(.077)$ & $.318(.075)$ & $.259(.06)$ & $.351(.071)$ & $.31(.063)$ & $-2.012(.943)$ & $-.297(.106)$ & \\
\hline M-VLDL-TG & $.205(.064)$ & $.248(.087)$ & $.3(.082)$ & $.224(.067)$ & $.275(.092)$ & $.246(.074)$ & $-2.133(.879)$ & $-.806(.455)$ & $\frac{0}{8}$ \\
\hline L-VLDL-C & $.299(.067)$ & $.304(.1)$ & $.297(.081)$ & $.291(.077)$ & $.289(.085)$ & $.317(.077)$ & $-1.254(.297)$ & $-.609(.337)$ & \\
\hline L-VLDL-CE & $.247(.061)$ & $.282(.088)$ & $.282(.082)$ & $.282(.072)$ & $.285(.082)$ & $.3(.112)$ & $-1.081(.282)$ & $-.673(.217)$ & \\
\hline L-VLDL-FC & $.316(.076)$ & $.294(.108)$ & $.311(.083)$ & $.287(.081)$ & $.351(.087)$ & $.298(.078)$ & $-1.274(.308)$ & $-.619(.291)$ & \\
\hline L-VLDL-L & NA & NA & $.36(.096)$ & NA & $.32(.102)$ & NA & $-1.277(.313)$ & $-.532(.278)$ & \\
\hline L-VLDL-P & $.268(.073)$ & $.287(.103)$ & $.281(.085)$ & $.262(.075)$ & $.219(.086)$ & $.255(.082)$ & $-1.357(.344)$ & $-.617(.229)$ & \\
\hline
\end{tabular}




\begin{tabular}{|c|c|c|c|c|c|c|c|c|c|}
\hline \multirow[b]{2}{*}{ Screening } & \multicolumn{8}{|c|}{ Method: RAPS/GRAPPLE + All SNPs } & \multirow{2}{*}{ 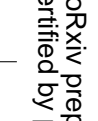 } \\
\hline & GERA & GERA & GERA & GLGC & Davis & Kettunen & GERA + Davis & GERA + Davis & \\
\hline Exposure & Davis & Davis & Kettunen & Davis & Kettunen & Davis & GLGC + Kettunen & GLGC + Kettunen & \\
\hline Outcome & CAD & UKB & UKB & UKB & UKB & UKB & $\mathrm{CAD}+\mathrm{UKB}$ & $\mathrm{CAD}+\mathrm{UKB}$ & \\
\hline Adjusted & & & & & & & $\mathrm{HDL}-\mathrm{C}+\mathrm{LDL}-\mathrm{C}+\mathrm{TG}$ & $\mathrm{ApoA} 1+\mathrm{ApoB}+\mathrm{TG}$ & \\
\hline L-VLDL-PL & $.322(.071)$ & $.318(.102)$ & $.346(.089)$ & $.283(.077)$ & $.397(.101)$ & $.351(.076)$ & $\mathrm{NaN}$ & $-.287(.104)$ & \\
\hline L-VLDL-TG & $.243(.077)$ & $.238(.104)$ & $.332(.094)$ & $.246(.08)$ & $.26(.103)$ & $.324(.082)$ & $-1.428(.372)$ & $-.252(.091)$ & \\
\hline XL-VLDL-L & NA & NA & $.289(.098)$ & NA & $.435(.14)$ & NA & $-1.069(.203)$ & $-.577(.249)$ & \\
\hline XL-VLDL-P & $.27(.074)$ & $.262(.099)$ & $.281(.093)$ & $.279(.084)$ & $.404(.122)$ & $.251(.084)$ & $-1.209(.238)$ & $-.373(.109)$ & \\
\hline XL-VLDL-PL & $.446(.09)$ & $.344(.13)$ & $.31(.093)$ & $.361(.118)$ & $.375(.12)$ & $.408(.102)$ & $-1.214(.257)$ & $-.583(.268)$ & \\
\hline XL-VLDL-TG & $.294(.092)$ & $.229(.109)$ & $.261(.094)$ & $.284(.095)$ & $.365(.111)$ & $.319(.093)$ & $-1.071(.205)$ & $-.603(.248)$ & \\
\hline XXL-VLDL-L & NA & NA & $.397(.108)$ & NA & $.312(.108)$ & NA & $-1.355(.318)$ & $-.402(.144)$ & \\
\hline XXL-VLDL-P & $.308(.08)$ & $.327(.096)$ & $.378(.097)$ & $.297(.088)$ & $.32(.101)$ & $.227(.073)$ & $-1.639(.502)$ & $-1.089(.449)$ & \\
\hline XXL-VLDL-PL & $.338(.091)$ & $.346(.103)$ & $.342(.103)$ & $.351(.103)$ & $.282(.114)$ & $.317(.086)$ & $-1.259(.262)$ & $-.814(.344)$ & \\
\hline XXL-VLDL-TG & $.384(.108)$ & $.374(.124)$ & $.348(.1)$ & $.433(.121)$ & $.304(.138)$ & $.359(.18)$ & $-1.202(.262)$ & $-1.075(.402)$ & \\
\hline \multicolumn{9}{|l|}{ IDL/LDL traits } & \\
\hline LDL-C & $.523(.043)$ & $.512(.053)$ & $.514(.042)$ & $.473(.055)$ & $.435(.048)$ & $.464(.048)$ & NA & $.319(.182)$ & \\
\hline ApoB & $.605(.056)$ & $.55(.062)$ & $.551(.052)$ & $.543(.069)$ & $.61(.066)$ & $.613(.06)$ & $-.532(.191)$ & NA & \\
\hline LDL-D & $.271(.215)$ & $.452(.299)$ & $2.064(.233)$ & $.831(.684)$ & $.328(.073)$ & $.201(.055)$ & $.145(.061)$ & $.119(.071)$ & \\
\hline S-LDL-C & $.624(.053)$ & $.589(.061)$ & $.539(.048)$ & $.537(.067)$ & $.474(.056)$ & $.48(.05)$ & $-.282(.152)$ & $.08(.238)$ & \\
\hline S-LDL-L & NA & NA & $.561(.047)$ & NA & $.473(.057)$ & NA & $-.251(.145)$ & $-.005(.29)$ & \\
\hline S-LDL-P & $.621(.057)$ & $.581(.065)$ & $.56(.049)$ & $.558(.073)$ & $.459(.061)$ & $.546(.063)$ & $-.266(.151)$ & $-.362(.596)$ & \\
\hline M-LDL-C & $.648(.055)$ & $.607(.062)$ & $.545(.044)$ & $.545(.068)$ & $.455(.049)$ & $.557(.054)$ & $-.271(.162)$ & -.169 (.909) & \\
\hline M-LDL-CE & $.643(.056)$ & $.601(.062)$ & $.564(.042)$ & $.545(.069)$ & $.467(.05)$ & $.55(.055)$ & $-.088(.188)$ & $\mathrm{NaN}$ & \\
\hline M-LDL-L & NA & NA & $.559(.042)$ & NA & $.461(.049)$ & NA & $-.069(.191)$ & $\mathrm{NaN}$ & \\
\hline M-LDL-P & $.638(.056)$ & $.597(.062)$ & $.557(.043)$ & $.54(.069)$ & $.472(.051)$ & $.46(.05)$ & $-.179(.174)$ & $.432(.31)$ & \\
\hline M-LDL-PL & $.658(.063)$ & $.605(.067)$ & $.556(.047)$ & $.571(.077)$ & $.506(.053)$ & $.559(.057)$ & $-.407(.162)$ & $-.566(.839)$ & \\
\hline L-LDL-C & $.627(.053)$ & $.577(.059)$ & $.515(.042)$ & $.504(.063)$ & $.465(.048)$ & $.488(.052)$ & $-.059(.261)$ & $\mathrm{NaN}$ & \\
\hline L-LDL-CE & $.638(.055)$ & $.589(.06)$ & $.555(.041)$ & $.514(.065)$ & $.463(.049)$ & $.493(.054)$ & $.116(.321)$ & $.461(.213)$ & \\
\hline L-LDL-FC & $.609(.051)$ & $.557(.057)$ & $.503(.041)$ & $.491(.06)$ & $.468(.047)$ & $.457(.052)$ & $.223(.315)$ & $\mathrm{NaN}$ & \\
\hline L-LDL-L & NA & NA & $.543(.04)$ & NA & $.468(.047)$ & NA & $.167(.273)$ & $\mathrm{NaN}$ & \\
\hline L-LDL-P & $.606(.052)$ & $.559(.058)$ & $.545(.041)$ & $.49(.062)$ & $.484(.046)$ & $.494(.048)$ & $.084(.213)$ & $\mathrm{NaN}$ & \\
\hline L-LDL-PL & $.61(.053)$ & $.558(.058)$ & $.515(.043)$ & $.492(.063)$ & $.528(.048)$ & $.502(.052)$ & $-.036(.195)$ & $\mathrm{NaN}$ & \\
\hline
\end{tabular}




\begin{tabular}{|c|c|c|c|c|c|c|c|c|c|}
\hline \multirow[b]{2}{*}{ Screening } & \multicolumn{8}{|c|}{ Method: RAPS/GRAPPLE + All SNPs } & \multirow{2}{*}{ 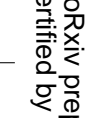 } \\
\hline & GERA & GERA & GERA & GLGC & Davis & Kettunen & GERA + Davis & GERA + Davis & \\
\hline Exposure & Davis & Davis & Kettunen & Davis & Kettunen & Davis & GLGC + Kettunen & GLGC + Kettunen & 霆 \\
\hline Outcome & $\mathrm{CAD}$ & UKB & UKB & UKB & UKB & UKB & $\mathrm{CAD}+\mathrm{UKB}$ & $\mathrm{CAD}+\mathrm{UKB}$ & $\stackrel{\Phi}{\Phi} \stackrel{\circ}{\circ}$ \\
\hline Adjusted & & & & & & & $\mathrm{HDL}-\mathrm{C}+\mathrm{LDL}-\mathrm{C}+\mathrm{TG}$ & $\mathrm{ApoA} 1+\mathrm{ApoB}+\mathrm{TG}$ & \\
\hline IDL-C & $.596(.054)$ & $.55(.059)$ & $.562(.042)$ & $.481(.064)$ & $.511(.047)$ & $.423(.051)$ & $.192(.229)$ & $.769(.501)$ & \\
\hline IDL-FC & $.586(.054)$ & $.539(.059)$ & $.525(.044)$ & $.494(.063)$ & $.44(.044)$ & $.402(.05)$ & $.19(.156)$ & $.33(.127)$ & \\
\hline IDL-L & NA & NA & $.57(.043)$ & NA & $.494(.048)$ & NA & $.148(.175)$ & $.444(.328)$ & \\
\hline IDL-P & $.566(.052)$ & $.536(.059)$ & $.575(.044)$ & $.488(.065)$ & $.434(.049)$ & $.412(.051)$ & $.153(.148)$ & $.292(.173)$ & \\
\hline IDL-PL & $.583(.052)$ & $.533(.058)$ & $.532(.045)$ & $.489(.064)$ & $.471(.047)$ & $.396(.05)$ & $.153(.18)$ & $.406(.184)$ & \\
\hline IDL-TG & $.603(.066)$ & $.595(.075)$ & $.658(.063)$ & $.567(.085)$ & $.432(.056)$ & $.315(.053)$ & $.11(.103)$ & $.047(.135)$ & \\
\hline \multicolumn{9}{|l|}{ HDL traits } & \multirow{10}{*}{ 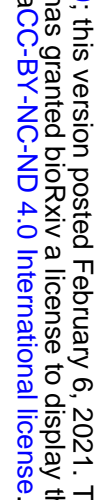 } \\
\hline HDL-C & $-.117(.031)$ & $-.199(.045)$ & $-.136(.055)$ & $-.317(.052)$ & $-.045(.059)$ & $-.108(.05)$ & NA & $\mathrm{NaN}$ & \\
\hline ApoA1 & $-.119(.042)$ & $-.193(.06)$ & $.023(.058)$ & $-.264(.071)$ & $.075(.064)$ & $-.13(.068)$ & $-.481(.271)$ & NA & \\
\hline HDL-D & $-.008(.027)$ & $-.124(.041)$ & $.004(.046)$ & $-.092(.048)$ & $.067(.045)$ & $.007(.041)$ & $.333(.114)$ & $.296(.1)$ & \\
\hline S-HDL-L & NA & NA & $-.098(.095)$ & NA & $-.037(.085)$ & NA & $-.312(.106)$ & $-.224(.087)$ & \\
\hline S-HDL-P & $-.265(.084)$ & $-.362(.113)$ & $-.13(.092)$ & $-.317(.119)$ & $-.053(.081)$ & $-.08(.094)$ & $-.331(.095)$ & $-.24(.083)$ & \\
\hline S-HDL-TG & $.354(.072)$ & $.386(.088)$ & $.65(.089)$ & $.475(.097)$ & $.351(.087)$ & $.283(.073)$ & $.253(.637)$ & $-.044(.466)$ & \\
\hline M-HDL-C & $-.323(.058)$ & $-.43(.079)$ & $-.364(.085)$ & $-.376(.091)$ & $-.46(.104)$ & $-.434(.075)$ & $-.508(.165)$ & $-.442(.143)$ & \\
\hline M-HDL-CE & $-.333(.058)$ & $-.458(.078)$ & $-.372(.09)$ & $-.385(.087)$ & $-.542(.105)$ & $-.443(.071)$ & $-.487(.157)$ & $-.413(.137)$ & \\
\hline M-HDL-FC & $-.275(.065)$ & $-.319(.08)$ & $-.262(.083)$ & $-.313(.092)$ & $-.313(.094)$ & $-.409(.082)$ & $-.649(.225)$ & $-.408(.166)$ & \\
\hline M-HDL-L & NA & NA & $-.311(.095)$ & NA & $-.474(.123)$ & NA & $-.606(.188)$ & $-.485(.155)$ & \\
\hline M-HDL-P & $-.298(.06)$ & $-.394(.086)$ & $-.273(.101)$ & $-.373(.1)$ & $-.565(.131)$ & -.307 (.079) & $-.694(.204)$ & $-.472(.166)$ & \\
\hline M-HDL-PL & $-.265(.058)$ & $-.346(.083)$ & $-.25(.09)$ & $-.335(.096)$ & $-.358(.104)$ & $-.3(.072)$ & $-.632(.191)$ & $-.486(.171)$ & \\
\hline L-HDL-C & $-.067(.03)$ & $-.144(.044)$ & $-.139(.051)$ & $-.144(.05)$ & $-.147(.052)$ & $-.049(.045)$ & $.516(.213)$ & $.575(.204)$ & 후음 \\
\hline L-HDL-CE & $-.063(.03)$ & $-.144(.044)$ & $-.116(.051)$ & $-.149(.051)$ & $-.134(.051)$ & $-.094(.047)$ & $.519(.23)$ & $.61(.206)$ & \\
\hline L-HDL-FC & $-.082(.03)$ & $-.144(.045)$ & $-.114(.053)$ & $-.128(.053)$ & $-.13(.051)$ & $-.03(.047)$ & $.518(.181)$ & $.59(.148)$ & \\
\hline L-HDL-L & $\mathrm{NA}$ & NA & $-.108(.05)$ & NA & $-.132(.052)$ & NA & $.457(.189)$ & $.541(.184)$ & \\
\hline L-HDL-P & $-.071(.028)$ & $-.146(.042)$ & $-.111(.05)$ & $-.13(.049)$ & $-.083(.05)$ & $-.1(.043)$ & $.422(.191)$ & $.476(.155)$ & \\
\hline L-HDL-PL & $-.087(.029)$ & $-.161(.043)$ & $-.141(.051)$ & $-.142(.051)$ & $-.105(.053)$ & $-.092(.044)$ & $.443(.202)$ & $.51(.169)$ & \\
\hline XL-HDL-C & $.055(.046)$ & $-.013(.068)$ & $.11(.066)$ & $.064(.073)$ & $.048(.069)$ & $.112(.068)$ & $.474(.223)$ & $.565(.196)$ & \\
\hline XL-HDL-CE & $.064(.044)$ & $.006(.066)$ & $.129(.066)$ & $.08(.07)$ & $.057(.068)$ & $.046(.075)$ & $.426(.177)$ & $.511(.206)$ & \\
\hline XL-HDL-FC & $.009(.039)$ & $-.05(.059)$ & $.066(.058)$ & $-.026(.067)$ & $.102(.06)$ & $.049(.066)$ & $.433(.16)$ & $.609(.159)$ & (1) \\
\hline
\end{tabular}




\begin{tabular}{|c|c|c|c|c|c|c|c|c|}
\hline \multirow[b]{2}{*}{ Screening } & \multicolumn{8}{|c|}{ Method: RAPS/GRAPPLE + All SNPs } \\
\hline & GERA & GERA & GERA & GLGC & Davis & Kettunen & GERA + Davis & GERA + Davis \\
\hline Exposure & Davis & Davis & Kettunen & Davis & Kettunen & Davis & GLGC + Kettunen & GLGC + Kettunen \\
\hline Outcome & CAD & UKB & UKB & UKB & UKB & UKB & $\mathrm{CAD}+\mathrm{UKB}$ & $\mathrm{CAD}+\mathrm{UKB}$ \\
\hline Adjusted & & & & & & & $\mathrm{HDL}-\mathrm{C}+\mathrm{LDL}-\mathrm{C}+\mathrm{TG}$ & $\mathrm{ApoA} 1+\mathrm{ApoB}+\mathrm{TG}$ \\
\hline XL-HDL-L & NA & NA & $.073(.055)$ & NA & $.038(.058)$ & NA & $.358(.154)$ & $.481(.141)$ \\
\hline XL-HDL-P & $.038(.033)$ & $-.022(.049)$ & $.112(.057)$ & $.017(.056)$ & $.083(.055)$ & $.023(.057)$ & $.41(.139)$ & $.39(.135)$ \\
\hline XL-HDL-PL & $.029(.031)$ & $-.031(.046)$ & $.037(.05)$ & $.005(.055)$ & $.038(.052)$ & $.013(.046)$ & $.343(.118)$ & $.466(.12)$ \\
\hline XL-HDL-TG & $.092(.027)$ & $.112(.041)$ & $.14(.047)$ & $.135(.047)$ & $.191(.042)$ & $.136(.039)$ & $.147(.074)$ & $.165(.086)$ \\
\hline
\end{tabular}




\section{Appendix 3.2 Univariable MR results}

Appendix 3-Table 3: Mendelian randomization results using genome-wide significant SNPs and inverse variance weighted (IVW) estimator.

\begin{tabular}{|c|c|c|c|c|c|c|}
\hline \multirow[b]{2}{*}{ Selection } & \multicolumn{6}{|c|}{ Method: IVW + Significant SNPs } \\
\hline & GERA & GERA & GERA & GLGC & Davis & Kettunen \\
\hline Exposure & Davis & Davis & Kettunen & Davis & Kettunen & Davis \\
\hline Outcome & CAD & UKB & UKB & UKB & UKB & UKB \\
\hline \multicolumn{7}{|l|}{ VLDL traits } \\
\hline TG & $.184(.051)$ & $.278(.076)$ & NA & $.309(.074)$ & NA & $.207(.064)$ \\
\hline VLDL-D & $.044(.06)$ & $.052(.09)$ & $.038(.102)$ & $.118(.091)$ & $-.083(.16)$ & $-.083(.138)$ \\
\hline XS-VLDL-L & NA & NA & $.353(.08)$ & NA & $.372(.083)$ & NA \\
\hline XS-VLDL-P & $.162(.04)$ & $.256(.059)$ & $.352(.081)$ & $.273(.063)$ & $.374(.084)$ & $.373(.095)$ \\
\hline XS-VLDL-PL & $.165(.046)$ & $.262(.069)$ & $.37(.088)$ & $.27(.075)$ & $.443(.048)$ & $.401(.07)$ \\
\hline XS-VLDL-TG & $.179(.041)$ & $.277(.061)$ & $.362(.082)$ & $.288(.062)$ & $.335(.076)$ & $.314(.08)$ \\
\hline S-VLDL-C & $.237(.053)$ & $.343(.08)$ & NA & $.339(.083)$ & NA & $.443(.116)$ \\
\hline S-VLDL-FC & $.21(.05)$ & $.307(.076)$ & $.344(.098)$ & $.314(.076)$ & $.262(.122)$ & $.397(.116)$ \\
\hline S-VLDL-L & NA & NA & $.318(.095)$ & NA & $.27(.106)$ & NA \\
\hline S-VLDL-P & $.188(.049)$ & $.274(.074)$ & $.311(.093)$ & $.29(.072)$ & $.266(.103)$ & $.331(.142)$ \\
\hline S-VLDL-PL & $.198(.048)$ & $.291(.072)$ & $.342(.091)$ & $.3(.072)$ & $.281(.089)$ & $.331(.125)$ \\
\hline S-VLDL-TG & $.174(.051)$ & $.255(.076)$ & $.296(.094)$ & $.28(.073)$ & $.261(.102)$ & $.262(.093)$ \\
\hline M-VLDL-C & $.188(.053)$ & $.265(.08)$ & $.305(.096)$ & $.287(.077)$ & $.361(.078)$ & $.32(.134)$ \\
\hline M-VLDL-CE & $.203(.051)$ & $.285(.077)$ & $.32(.098)$ & $.295(.076)$ & $.264(.094)$ & $.291(.125)$ \\
\hline M-VLDL-FC & $.165(.056)$ & $.233(.084)$ & $.292(.098)$ & $.27(.08)$ & $.3(.084)$ & $.303(.104)$ \\
\hline M-VLDL-L & NA & NA & $.265(.104)$ & NA & $.357(.096)$ & NA \\
\hline M-VLDL-P & $.153(.056)$ & $.214(.085)$ & $.276(.104)$ & $.258(.081)$ & $.322(.092)$ & $.268(.074)$ \\
\hline M-VLDL-PL & $.163(.054)$ & $.23(.082)$ & $.296(.097)$ & $.266(.078)$ & $.302(.084)$ & $.289(.095)$ \\
\hline M-VLDL-TG & $.14(.058)$ & $.196(.087)$ & $.268(.107)$ & $.247(.083)$ & $.327(.093)$ & $.245(.091)$ \\
\hline L-VLDL-C & $.177(.06)$ & $.24(.091)$ & $.288(.106)$ & $.286(.089)$ & $.108(.223)$ & $.31(.084)$ \\
\hline L-VLDL-CE & $.178(.057)$ & $.245(.087)$ & $.262(.105)$ & $.279(.086)$ & $.182(.187)$ & $.299(.077)$ \\
\hline L-VLDL-FC & $.176(.063)$ & $.242(.094)$ & $.295(.108)$ & $.298(.091)$ & $.321(.101)$ & $.314(.082)$ \\
\hline L-VLDL-L & NA & NA & $.291(.119)$ & NA & $.125(.232)$ & NA \\
\hline L-VLDL-P & $.164(.062)$ & $.227(.093)$ & $.269(.108)$ & $.275(.09)$ & $.332(.127)$ & $.247(.076)$ \\
\hline L-VLDL-PL & $.173(.061)$ & $.23(.092)$ & $.308(.115)$ & $.284(.088)$ & $.32(.127)$ & $.302(.079)$ \\
\hline
\end{tabular}

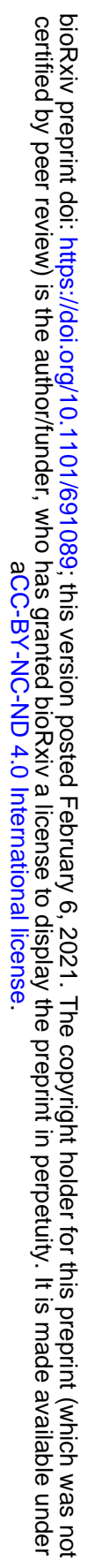




\begin{tabular}{|c|c|c|c|c|c|c|}
\hline \multirow[b]{2}{*}{ Selection } & \multicolumn{6}{|c|}{ Method: IVW + Significant SNPs } \\
\hline & GERA & GERA & GERA & GLGC & Davis & Kettunen \\
\hline Exposure & Davis & Davis & Kettunen & Davis & Kettunen & Davis \\
\hline Outcome & CAD & UKB & UKB & UKB & UKB & UKB \\
\hline L-VLDL-TG & $.149(.063)$ & $.202(.095)$ & $.268(.118)$ & $.267(.092)$ & $.33(.131)$ & $.302(.08)$ \\
\hline XL-VLDL-L & NA & NA & $.263(.123)$ & NA & $.365(.286)$ & NA \\
\hline XL-VLDL-P & $.149(.063)$ & $.206(.095)$ & $.247(.122)$ & $.268(.096)$ & $.346(.28)$ & $.245(.077)$ \\
\hline XL-VLDL-PL & $.176(.067)$ & $.243(.101)$ & $.292(.119)$ & $.323(.101)$ & $.333(.265)$ & $.344(.133)$ \\
\hline XL-VLDL-TG & $.151(.066)$ & $.205(.1)$ & $.241(.12)$ & $.282(.1)$ & $.323(.272)$ & $.249(.081)$ \\
\hline XXL-VLDL-L & NA & NA & $.356(.127)$ & NA & $-.165(.425)$ & NA \\
\hline XXL-VLDL-P & $.228(.067)$ & $.35(.099)$ & $.372(.119)$ & $.376(.098)$ & $-.12(.389)$ & $.006(.153)$ \\
\hline XXL-VLDL-PL & $.211(.07)$ & $.31(.105)$ & $.275(.125)$ & $.399(.107)$ & $-.145(.395)$ & $.071(.191)$ \\
\hline XXL-VLDL-TG & $.221(.067)$ & $.3(.102)$ & $.292(.126)$ & $.415(.104)$ & $.09(.36)$ & $.349(.303)$ \\
\hline \multicolumn{7}{|l|}{ IDL/LDL traits } \\
\hline LDL-C & $.427(.049)$ & $.431(.054)$ & $.409(.077)$ & $.409(.054)$ & $.416(.099)$ & $.422(.063)$ \\
\hline ApoB & $.506(.058)$ & $.525(.065)$ & $.474(.093)$ & $.473(.064)$ & $.636(.092)$ & $.569(.071)$ \\
\hline LDL-D & $.217(.151)$ & $.423(.161)$ & $1.121(.178)$ & $.271(.143)$ & $.309(.126)$ & $.211(.081)$ \\
\hline S-LDL-C & $.481(.056)$ & $.467(.063)$ & $.445(.087)$ & $.438(.063)$ & $.44(.128)$ & $.436(.076)$ \\
\hline S-LDL-L & NA & NA & $.44(.09)$ & NA & $.456(.132)$ & NA \\
\hline S-LDL-P & $.501(.059)$ & $.494(.068)$ & $.449(.093)$ & $.472(.067)$ & $.49(.139)$ & $.588(.097)$ \\
\hline M-LDL-C & $.475(.057)$ & $.457(.064)$ & $.426(.08)$ & $.427(.064)$ & $.418(.111)$ & $.436(.087)$ \\
\hline M-LDL-CE & $.485(.058)$ & $.47(.065)$ & $.432(.078)$ & $.436(.064)$ & $.43(.107)$ & $.444(.085)$ \\
\hline M-LDL-L & NA & NA & $.43(.08)$ & NA & $.43(.11)$ & NA \\
\hline M-LDL-P & $.479(.057)$ & $.465(.064)$ & $.437(.081)$ & $.44(.064)$ & $.413(.122)$ & $.439(.093)$ \\
\hline M-LDL-PL & $.5(.063)$ & $.49(.071)$ & $.437(.087)$ & $.464(.07)$ & $.443(.132)$ & $.497(.099)$ \\
\hline L-LDL-C & $.449(.055)$ & $.436(.061)$ & $.432(.076)$ & $.411(.061)$ & $.409(.106)$ & $.417(.076)$ \\
\hline L-LDL-CE & $.464(.056)$ & $.451(.062)$ & $.426(.075)$ & $.422(.062)$ & $.416(.102)$ & $.433(.077)$ \\
\hline L-LDL-FC & $.425(.054)$ & $.411(.059)$ & $.424(.074)$ & $.393(.059)$ & $.387(.105)$ & $.394(.078)$ \\
\hline L-LDL-L & NA & NA & $.427(.074)$ & NA & $.407(.103)$ & NA \\
\hline L-LDL-P & $.448(.054)$ & $.442(.06)$ & $.435(.075)$ & $.421(.059)$ & $.413(.104)$ & $.424(.075)$ \\
\hline L-LDL-PL & $.444(.056)$ & $.438(.061)$ & $.441(.078)$ & $.423(.061)$ & $.42(.109)$ & $.429(.076)$ \\
\hline IDL-C & $.447(.055)$ & $.455(.059)$ & $.451(.075)$ & $.433(.06)$ & $.439(.085)$ & $.422(.07)$ \\
\hline IDL-FC & $.429(.055)$ & $.439(.059)$ & $.468(.075)$ & $.414(.059)$ & $.431(.081)$ & $.402(.074)$ \\
\hline
\end{tabular}




\begin{tabular}{|c|c|c|c|c|c|c|}
\hline \multirow[b]{2}{*}{ Selection } & \multicolumn{6}{|c|}{ Method: IVW + Significant SNPs } \\
\hline & GERA & GERA & GERA & GLGC & Davis & Kettunen \\
\hline Exposure & Davis & Davis & Kettunen & Davis & Kettunen & Davis \\
\hline Outcome & CAD & UKB & UKB & UKB & UKB & UKB \\
\hline IDL-L & NA & NA & $.467(.075)$ & NA & $.445(.085)$ & NA \\
\hline IDL-P & $.443(.055)$ & $.467(.06)$ & $.48(.077)$ & $.45(.059)$ & $.446(.088)$ & $.426(.071)$ \\
\hline IDL-PL & $.429(.055)$ & $.443(.059)$ & $.473(.078)$ & $.427(.059)$ & $.435(.092)$ & $.407(.069)$ \\
\hline IDL-TG & $.461(.07)$ & $.518(.076)$ & $.625(.098)$ & $.494(.073)$ & $.342(.085)$ & $.34(.123)$ \\
\hline \multicolumn{7}{|l|}{ HDL traits } \\
\hline HDL-C & $-.085(.044)$ & $-.156(.057)$ & $-.146(.085)$ & $-.195(.06)$ & $-.082(.159)$ & $-.015(.109)$ \\
\hline ApoA1 & $-.072(.054)$ & $-.155(.071)$ & -.036 (.09) & $-.194(.074)$ & $.001(.192)$ & $.066(.158)$ \\
\hline HDL-D & $-.027(.042)$ & $-.071(.058)$ & $-.052(.073)$ & $-.092(.063)$ & $.073(.098)$ & $.074(.074)$ \\
\hline S-HDL-L & NA & NA & $-.064(.148)$ & NA & $-.033(.092)$ & NA \\
\hline S-HDL-P & $-.117(.087)$ & $-.172(.116)$ & $-.13(.146)$ & $-.298(.117)$ & $-.033(.09)$ & $-.115(.174)$ \\
\hline S-HDL-TG & $.224(.063)$ & $.317(.082)$ & $.496(.107)$ & $.344(.085)$ & $.334(.096)$ & $.286(.17)$ \\
\hline M-HDL-C & $-.214(.062)$ & $-.327(.078)$ & $-.48(.111)$ & $-.39(.079)$ & $-.423(.175)$ & $-.39(.159)$ \\
\hline M-HDL-CE & $-.227(.062)$ & $-.338(.077)$ & $-.497(.111)$ & $-.4(.078)$ & $-.435(.194)$ & $-.341(.238)$ \\
\hline M-HDL-FC & $-.158(.065)$ & $-.272(.084)$ & $-.341(.117)$ & $-.337(.085)$ & $-.288(.218)$ & $-.278(.144)$ \\
\hline M-HDL-L & NA & NA & $-.436(.125)$ & NA & $-.514(.223)$ & NA \\
\hline M-HDL-P & $-.172(.066)$ & $-.292(.087)$ & $-.414(.132)$ & $-.361(.089)$ & $-.386(.307)$ & $-.18(.118)$ \\
\hline M-HDL-PL & $-.161(.064)$ & $-.275(.085)$ & $-.38(.126)$ & $-.345(.087)$ & $-.419(.301)$ & $-.2(.099)$ \\
\hline L-HDL-C & $-.047(.044)$ & $-.097(.059)$ & $-.124(.08)$ & $-.133(.063)$ & $.022(.106)$ & $.021(.105)$ \\
\hline L-HDL-CE & $-.049(.044)$ & -.098 (.059) & $-.12(.079)$ & $-.137(.063)$ & $.023(.112)$ & $.004(.106)$ \\
\hline L-HDL-FC & $-.044(.046)$ & $-.094(.062)$ & $-.106(.082)$ & $-.127(.067)$ & $.038(.103)$ & $.017(.109)$ \\
\hline L-HDL-L & NA & NA & $-.106(.077)$ & NA & $.034(.102)$ & NA \\
\hline L-HDL-P & $-.045(.043)$ & $-.097(.058)$ & $-.102(.077)$ & $-.125(.063)$ & $.009(.111)$ & $.025(.11)$ \\
\hline L-HDL-PL & $-.054(.044)$ & $-.11(.06)$ & $-.115(.079)$ & $-.14(.064)$ & $.006(.115)$ & $.016(.115)$ \\
\hline XL-HDL-C & $.03(.06)$ & $-.012(.084)$ & $.014(.099)$ & $-.05(.088)$ & $-.015(.165)$ & $.161(.101)$ \\
\hline XL-HDL-CE & $.03(.059)$ & $-.009(.081)$ & $.025(.098)$ & $-.042(.086)$ & $-.001(.166)$ & $.221(.107)$ \\
\hline XL-HDL-FC & $-.003(.056)$ & $-.05(.076)$ & $-.001(.089)$ & $-.077(.081)$ & $.072(.11)$ & $.057(.092)$ \\
\hline XL-HDL-L & NA & NA & $.001(.085)$ & NA & $-.009(.138)$ & NA \\
\hline XL-HDL-P & $.015(.049)$ & $-.021(.067)$ & $.013(.088)$ & $-.042(.071)$ & $.103(.1)$ & $.135(.093)$ \\
\hline XL-HDL-PL & $0(.047)$ & $-.037(.065)$ & $-.026(.079)$ & $-.055(.069)$ & $.081(.088)$ & $.071(.069)$ \\
\hline
\end{tabular}




\begin{tabular}{lcccccc}
\hline & \multicolumn{7}{c}{ Method: IVW + Significant SNPs } \\
\cline { 2 - 7 } Selection & GERA & GERA & GERA & GLGC & Davis & Kettunen \\
Exposure & Davis & Davis & Kettunen & Davis & Kettunen & Davis \\
Outcome & CAD & UKB & UKB & UKB & UKB & UKB \\
\hline XL-HDL-TG & $.086(.041)$ & $.103(.059)$ & $.14(.075)$ & $.13(.063)$ & $.165(.043)$ & $.126(.051)$ \\
\hline
\end{tabular}


Appendix 3-Table 4: Mendelian randomization results using genome-wide significant SNPs and the weighted median estimator.

\begin{tabular}{|c|c|c|c|c|c|c|}
\hline \multirow[b]{2}{*}{ Selection } & \multicolumn{6}{|c|}{ Method: Weighted median + Significant SNPs } \\
\hline & GERA & GERA & GERA & GLGC & Davis & Kettunen \\
\hline Exposure & Davis & Davis & Kettunen & Davis & Kettunen & Davis \\
\hline Outcome & CAD & UKB & UKB & UKB & UKB & UKB \\
\hline \multicolumn{7}{|l|}{ VLDL traits } \\
\hline TG & $.042(.055)$ & $.191(.072)$ & NA & $.228(.069)$ & NA & $.195(.077)$ \\
\hline VLDL-D & $-.098(.052)$ & $.039(.095)$ & $.057(.11)$ & $.058(.093)$ & $-.107(.099)$ & $-.052(.115)$ \\
\hline XS-VLDL-L & NA & NA & $.312(.076)$ & NA & $.393(.078)$ & NA \\
\hline XS-VLDL-P & $.101(.037)$ & $.23(.052)$ & $.303(.079)$ & $.229(.052)$ & $.409(.08)$ & $.253(.059)$ \\
\hline XS-VLDL-PL & $.096(.039)$ & $.242(.059)$ & $.352(.087)$ & $.228(.06)$ & $.422(.065)$ & $.319(.062)$ \\
\hline XS-VLDL-TG & $.125(.041)$ & $.266(.057)$ & $.287(.079)$ & $.221(.056)$ & $.361(.084)$ & $.306(.069)$ \\
\hline S-VLDL-C & $.187(.059)$ & $.232(.075)$ & NA & $.256(.074)$ & NA & $.303(.094)$ \\
\hline S-VLDL-FC & $.152(.057)$ & $.207(.069)$ & $.289(.093)$ & $.227(.069)$ & $.316(.109)$ & $.279(.077)$ \\
\hline S-VLDL-L & NA & NA & $.282(.083)$ & NA & $.306(.099)$ & NA \\
\hline S-VLDL-P & $.131(.057)$ & $.202(.069)$ & $.275(.085)$ & $.221(.062)$ & $.291(.093)$ & $.226(.078)$ \\
\hline S-VLDL-PL & $.137(.053)$ & $.205(.067)$ & $.283(.083)$ & $.218(.062)$ & $.305(.092)$ & $.263(.075)$ \\
\hline S-VLDL-TG & $.112(.057)$ & $.204(.067)$ & $.216(.088)$ & $.229(.064)$ & $.267(.099)$ & $.244(.073)$ \\
\hline M-VLDL-C & $.12(.058)$ & $.2(.07)$ & $.255(.088)$ & $.213(.066)$ & $.303(.099)$ & $.224(.081)$ \\
\hline M-VLDL-CE & $.144(.054)$ & $.207(.071)$ & $.262(.087)$ & $.207(.068)$ & $.301(.098)$ & $.209(.072)$ \\
\hline M-VLDL-FC & $.081(.058)$ & $.188(.074)$ & $.221(.087)$ & $.218(.068)$ & $.272(.102)$ & $.231(.08)$ \\
\hline M-VLDL-L & NA & NA & $.227(.095)$ & NA & $.275(.109)$ & NA \\
\hline M-VLDL-P & $.047(.06)$ & $.191(.072)$ & $.221(.096)$ & $.226(.069)$ & $.31(.104)$ & $.257(.079)$ \\
\hline M-VLDL-PL & $.103(.056)$ & $.197(.071)$ & $.228(.089)$ & $.217(.064)$ & $.29(.104)$ & $.231(.078)$ \\
\hline M-VLDL-TG & $-.005(.06)$ & $.199(.075)$ & $.224(.089)$ & $.222(.068)$ & $.318(.113)$ & $.233(.085)$ \\
\hline L-VLDL-C & $.109(.068)$ & $.2(.078)$ & $.237(.093)$ & $.231(.075)$ & $.242(.122)$ & $.262(.088)$ \\
\hline L-VLDL-CE & $.147(.063)$ & $.211(.079)$ & $.249(.09)$ & $.253(.073)$ & $.281(.11)$ & $.286(.081)$ \\
\hline L-VLDL-FC & $.045(.065)$ & $.199(.085)$ & $.225(.093)$ & $.224(.077)$ & $.252(.125)$ & $.228(.089)$ \\
\hline L-VLDL-L & NA & NA & $.243(.102)$ & NA & $.261(.122)$ & NA \\
\hline L-VLDL-P & $.041(.064)$ & $.209(.082)$ & $.224(.092)$ & $.21(.079)$ & $.289(.122)$ & $.223(.086)$ \\
\hline L-VLDL-PL & $.08(.063)$ & $.201(.08)$ & $.244(.101)$ & $.224(.077)$ & $.278(.123)$ & $.247(.092)$ \\
\hline L-VLDL-TG & $-.008(.061)$ & $.215(.084)$ & $.225(.103)$ & $.161(.077)$ & $.286(.13)$ & $.277(.093)$ \\
\hline XL-VLDL-L & NA & NA & $.262(.111)$ & NA & NA & NA \\
\hline
\end{tabular}




\begin{tabular}{|c|c|c|c|c|c|c|}
\hline \multirow[b]{2}{*}{ Selection } & \multicolumn{6}{|c|}{ Method: Weighted median + Significant SNPs } \\
\hline & GERA & GERA & GERA & GLGC & Davis & Kettunen \\
\hline Exposure & Davis & Davis & Kettunen & Davis & Kettunen & Davis \\
\hline Outcome & CAD & UKB & UKB & UKB & UKB & UKB \\
\hline XL-VLDL-P & $-.026(.063)$ & $.207(.091)$ & $.289(.102)$ & $.192(.088)$ & NA & $.209(.101)$ \\
\hline XL-VLDL-PL & $-.006(.067)$ & $.197(.094)$ & $.253(.094)$ & $.213(.088)$ & NA & $.24(.101)$ \\
\hline XL-VLDL-TG & $-.026(.064)$ & $.214(.092)$ & $.229(.102)$ & $.191(.088)$ & NA & $.212(.099)$ \\
\hline XXL-VLDL-L & NA & NA & $.316(.114)$ & NA & $-.156(.22)$ & NA \\
\hline XXL-VLDL-P & $.091(.071)$ & $.236(.089)$ & $.267(.1)$ & $.263(.088)$ & $-.104(.173)$ & $.185(.098)$ \\
\hline XXL-VLDL-PL & $.153(.082)$ & $.283(.096)$ & $.267(.11)$ & $.332(.095)$ & $-.139(.178)$ & $.126(.124)$ \\
\hline XXL-VLDL-TG & $.126(.078)$ & $.266(.096)$ & $.244(.108)$ & $.339(.097)$ & $.227(.171)$ & $.23(.123)$ \\
\hline \multicolumn{7}{|l|}{ IDL/LDL traits } \\
\hline LDL-C & $.263(.053)$ & $.307(.066)$ & $.274(.05)$ & $.297(.063)$ & $.435(.072)$ & $.431(.067)$ \\
\hline ApoB & $.365(.073)$ & $.472(.078)$ & $.381(.063)$ & $.375(.081)$ & $.624(.08)$ & $.565(.094)$ \\
\hline LDL-D & $.306(.09)$ & $.413(.157)$ & $.467(.163)$ & $.271(.142)$ & $.294(.075)$ & $.193(.06)$ \\
\hline S-LDL-C & $.271(.058)$ & $.342(.073)$ & $.343(.056)$ & $.273(.068)$ & $.498(.08)$ & $.274(.083)$ \\
\hline S-LDL-L & NA & NA & $.354(.061)$ & NA & $.449(.081)$ & NA \\
\hline S-LDL-P & $.355(.063)$ & $.366(.078)$ & $.397(.069)$ & $.329(.08)$ & $.49(.089)$ & $.581(.098)$ \\
\hline M-LDL-C & $.283(.055)$ & $.313(.073)$ & $.299(.05)$ & $.244(.07)$ & $.474(.074)$ & $.297(.074)$ \\
\hline M-LDL-CE & $.27(.055)$ & $.333(.077)$ & $.299(.051)$ & $.255(.071)$ & $.437(.081)$ & $.311(.077)$ \\
\hline M-LDL-L & NA & NA & $.303(.053)$ & NA & $.432(.079)$ & NA \\
\hline M-LDL-P & $.251(.057)$ & $.32(.071)$ & $.309(.054)$ & $.278(.07)$ & $.409(.072)$ & $.325(.078)$ \\
\hline M-LDL-PL & $.343(.063)$ & $.337(.081)$ & $.316(.055)$ & $.318(.078)$ & $.457(.074)$ & $.353(.085)$ \\
\hline L-LDL-C & $.251(.052)$ & $.29(.067)$ & $.303(.048)$ & $.231(.063)$ & $.45(.075)$ & $.309(.071)$ \\
\hline L-LDL-CE & $.251(.054)$ & $.32(.068)$ & $.293(.052)$ & $.241(.066)$ & $.481(.074)$ & $.322(.077)$ \\
\hline L-LDL-FC & $.251(.048)$ & $.214(.061)$ & $.301(.049)$ & $.214(.062)$ & $.427(.068)$ & $.289(.065)$ \\
\hline L-LDL-L & NA & NA & $.289(.051)$ & NA & $.412(.07)$ & NA \\
\hline L-LDL-P & $.281(.053)$ & $.321(.067)$ & $.29(.053)$ & $.244(.066)$ & $.42(.072)$ & $.351(.072)$ \\
\hline L-LDL-PL & $.286(.05)$ & $.32(.067)$ & $.313(.052)$ & $.298(.065)$ & $.413(.074)$ & $.35(.076)$ \\
\hline IDL-C & $.283(.056)$ & $.349(.068)$ & $.315(.053)$ & $.313(.07)$ & $.51(.072)$ & $.383(.068)$ \\
\hline IDL-FC & $.283(.053)$ & $.334(.066)$ & $.337(.053)$ & $.314(.065)$ & $.422(.067)$ & $.367(.064)$ \\
\hline IDL-L & NA & NA & $.329(.056)$ & NA & $.494(.069)$ & NA \\
\hline IDL-P & $.331(.06)$ & $.44(.067)$ & $.343(.056)$ & $.371(.069)$ & $.463(.074)$ & $.328(.068)$ \\
\hline
\end{tabular}

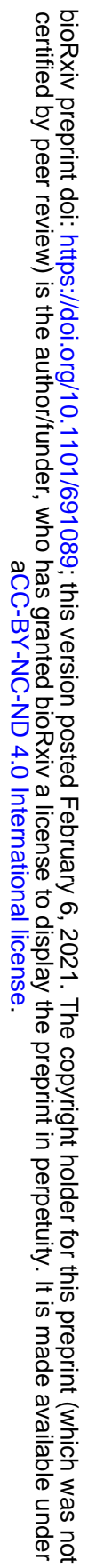




\begin{tabular}{|c|c|c|c|c|c|c|}
\hline \multirow[b]{2}{*}{ Selection } & \multicolumn{6}{|c|}{ Method: Weighted median + Significant SNPs } \\
\hline & GERA & GERA & GERA & GLGC & Davis & Kettunen \\
\hline Exposure & Davis & Davis & Kettunen & Davis & Kettunen & Davis \\
\hline Outcome & CAD & UKB & UKB & UKB & UKB & UKB \\
\hline IDL-PL & $.265(.055)$ & $.332(.066)$ & $.344(.056)$ & $.316(.066)$ & $.451(.072)$ & $.359(.066)$ \\
\hline IDL-TG & $.233(.067)$ & $.371(.086)$ & $.605(.078)$ & $.337(.085)$ & $.315(.082)$ & $.215(.057)$ \\
\hline \multicolumn{7}{|l|}{ HDL traits } \\
\hline HDL-C & $-.017(.04)$ & $-.167(.058)$ & $-.17(.072)$ & $-.167(.058)$ & $-.096(.077)$ & $-.085(.07)$ \\
\hline ApoA1 & $.094(.049)$ & $-.06(.076)$ & $-.069(.087)$ & $-.167(.07)$ & $.005(.083)$ & $-.051(.121)$ \\
\hline HDL-D & $.079(.034)$ & $.062(.061)$ & $.102(.064)$ & $.088(.061)$ & $.099(.061)$ & $.096(.058)$ \\
\hline S-HDL-L & NA & NA & $-.174(.113)$ & NA & NA & NA \\
\hline S-HDL-P & -.173(.069) & $.018(.106)$ & $-.171(.109)$ & $-.235(.113)$ & NA & $-.049(.108)$ \\
\hline S-HDL-TG & $.157(.061)$ & $.238(.085)$ & $.312(.105)$ & $.228(.086)$ & $.327(.105)$ & $.229(.076)$ \\
\hline M-HDL-C & $-.169(.054)$ & $-.236(.082)$ & $-.264(.097)$ & $-.241(.077)$ & $-.392(.098)$ & $-.266(.084)$ \\
\hline M-HDL-CE & $-.166(.053)$ & $-.23(.08)$ & $-.271(.099)$ & $-.238(.075)$ & $-.394(.103)$ & $-.23(.085)$ \\
\hline M-HDL-FC & $-.166(.055)$ & $-.254(.086)$ & $-.281(.098)$ & $-.282(.087)$ & $-.28(.102)$ & $-.22(.1)$ \\
\hline M-HDL-L & NA & NA & $-.296(.113)$ & NA & $-.448(.122)$ & NA \\
\hline M-HDL-P & $-.157(.056)$ & -.199 (.09) & $-.298(.112)$ & $-.231(.086)$ & $-.291(.136)$ & $-.165(.131)$ \\
\hline M-HDL-PL & $-.143(.058)$ & $-.183(.088)$ & $-.285(.108)$ & $-.183(.085)$ & $-.321(.114)$ & $-.203(.12)$ \\
\hline L-HDL-C & $.086(.037)$ & $-.009(.066)$ & $.031(.083)$ & $-.032(.08)$ & $.003(.09)$ & $.006(.068)$ \\
\hline L-HDL-CE & $.086(.038)$ & $-.011(.067)$ & $.075(.077)$ & $-.037(.076)$ & $.015(.091)$ & $-.006(.068)$ \\
\hline L-HDL-FC & $.09(.039)$ & $-.005(.067)$ & $.079(.081)$ & $-.019(.076)$ & $.041(.078)$ & $.027(.074)$ \\
\hline L-HDL-L & NA & NA & $.074(.077)$ & NA & $.068(.084)$ & NA \\
\hline L-HDL-P & $.081(.036)$ & $.046(.062)$ & $.075(.074)$ & $-.01(.066)$ & $.066(.07)$ & $.078(.064)$ \\
\hline L-HDL-PL & $.084(.039)$ & $0(.067)$ & $.051(.082)$ & $-.021(.071)$ & $.054(.075)$ & $.074(.071)$ \\
\hline XL-HDL-C & $.163(.047)$ & $.122(.091)$ & $.136(.087)$ & $.132(.09)$ & $.02(.098)$ & $.161(.096)$ \\
\hline XL-HDL-CE & $.139(.044)$ & $.106(.088)$ & $.122(.09)$ & $.148(.085)$ & $.038(.091)$ & $.336(.092)$ \\
\hline XL-HDL-FC & $.135(.048)$ & $.065(.079)$ & $.133(.081)$ & $.027(.077)$ & $.159(.079)$ & $.052(.086)$ \\
\hline XL-HDL-L & $\mathrm{NA}$ & NA & $.119(.075)$ & $\mathrm{NA}$ & $.023(.078)$ & NA \\
\hline XL-HDL-P & $.115(.035)$ & $.087(.07)$ & $.12(.073)$ & $.129(.067)$ & $.16(.071)$ & $.15(.073)$ \\
\hline XL-HDL-PL & $.101(.037)$ & $.064(.07)$ & $.11(.072)$ & $.121(.069)$ & $.141(.069)$ & $.088(.065)$ \\
\hline XL-HDL-TG & $.074(.027)$ & $.107(.047)$ & $.126(.051)$ & $.118(.042)$ & $.156(.05)$ & $.114(.045)$ \\
\hline
\end{tabular}




\section{Appendix 3.3 Multivariable MR results}

Appendix 3-Table 5: Multivariable Mendelian randomization results (adjusted for HDL-C, LDL-C, and TG).

\begin{tabular}{|c|c|c|c|c|}
\hline Trait & HDL-C & LDL-C & TG & Subfraction \\
\hline \multicolumn{5}{|l|}{ VLDL traits } \\
\hline VLDL-D & $-.251(.052)$ & $.29(.037)$ & $.6(.087)$ & $-.588(.094)$ \\
\hline XS-VLDL-L & $-.086(.046)$ & $.286(.077)$ & $.089(.099)$ & $.132(.119)$ \\
\hline XS-VLDL-P & $-.083(.045)$ & $.299(.078)$ & $.093(.106)$ & $.118(.125)$ \\
\hline XS-VLDL-PL & $-.083(.046)$ & $.249(.098)$ & $.112(.076)$ & $.159(.12)$ \\
\hline XS-VLDL-TG & $-.114(.046)$ & $.463(.079)$ & $.286(.173)$ & $-.157(.187)$ \\
\hline S-VLDL-C & $-.267(.084)$ & $.754(.112)$ & $1.033(.28)$ & $-1.035(.323)$ \\
\hline S-VLDL-FC & $-.195(.068)$ & $.898(.163)$ & $.935(.26)$ & $-1.027(.337)$ \\
\hline S-VLDL-L & $-.25(.072)$ & $.755(.112)$ & $.876(.233)$ & $-.898(.28)$ \\
\hline S-VLDL-P & $-.31(.101)$ & $.819(.157)$ & $1.209(.4)$ & $-1.245(.463)$ \\
\hline S-VLDL-PL & $-.168(.051)$ & $.673(.074)$ & $.626(.159)$ & $-.613(.182)$ \\
\hline S-VLDL-TG & $-.499(.305)$ & $.906(.34)$ & $2.532(1.57)$ & $-2.628(1.741)$ \\
\hline M-VLDL-C & $-.201(.068)$ & $.808(.127)$ & $1.472(.424)$ & $-1.433(.451)$ \\
\hline M-VLDL-CE & $-.168(.061)$ & & $.996(.249)$ & $-1.035(.293)$ \\
\hline M-VLDL-FC & $-.2(.072)$ & $.658(.089)$ & $1.469(.417)$ & $-1.412(.444)$ \\
\hline M-VLDL-L & $-.355(.139)$ & $.602(.096)$ & $1.787(.654)$ & $-1.878(.75)$ \\
\hline M-VLDL-P & $-.362(.124)$ & $.569(.08)$ & $1.889(.676)$ & $-1.974(.745)$ \\
\hline M-VLDL-PL & $-.332(.141)$ & $.722(.159)$ & $1.996(.869)$ & $-2.012(.943)$ \\
\hline M-VLDL-TG & $-.408(.153)$ & $.432(.061)$ & $1.974(.772)$ & $-2.133(.879)$ \\
\hline L-VLDL-C & $-.216(.063)$ & $.509(.046)$ & $1.163(.254)$ & $-1.254(.297)$ \\
\hline L-VLDL-CE & $-.272(.072)$ & $.465(.04)$ & $1.038(.242)$ & $-1.081(.282)$ \\
\hline L-VLDL-FC & $-.144(.059)$ & $.493(.044)$ & $1.233(.27)$ & $-1.274(.308)$ \\
\hline L-VLDL-L & $-.228(.066)$ & $.414(.045)$ & $1.17(.263)$ & $-1.277(.313)$ \\
\hline L-VLDL-P & $-.115(.056)$ & $.442(.046)$ & $1.351(.317)$ & $-1.357(.344)$ \\
\hline L-VLDL-PL & $-.221(.111)$ & $.473(.07)$ & $2.135(.948)$ & $-2.316(1.112)$ \\
\hline L-VLDL-TG & $-.196(.066)$ & $.355(.05)$ & $1.357(.322)$ & $-1.428(.372)$ \\
\hline XL-VLDL-L & $-.126(.049)$ & $.451(.04)$ & $.896(.159)$ & $-1.069(.203)$ \\
\hline XL-VLDL-P & $-.127(.053)$ & $.474(.043)$ & $1.038(.183)$ & $-1.209(.238)$ \\
\hline XL-VLDL-PL & $-.138(.055)$ & $.5(.044)$ & $1.052(.204)$ & $-1.214(.257)$ \\
\hline XL-VLDL-TG & $-.129(.049)$ & $.424(.04)$ & $.944(.167)$ & $-1.071(.205)$ \\
\hline XXL-VLDL-L & $-.228(.067)$ & $.444(.043)$ & $.978(.207)$ & $-1.355(.318)$ \\
\hline XXL-VLDL-P & $.063(.076)$ & $.452(.05)$ & $1.371(.384)$ & $-1.639(.502)$ \\
\hline XXL-VLDL-PL & $-.185(.056)$ & $.371(.042)$ & $.997(.185)$ & $-1.259(.262)$ \\
\hline XXL-VLDL-TG & $-.152(.059)$ & $.41(.04)$ & $.966(.19)$ & $-1.202(.262)$ \\
\hline \multicolumn{5}{|l|}{ LDL/IDL traits } \\
\hline ApoB & $-.084(.046)$ & $.8(.146)$ & $.427(.101)$ & $-.532(.191)$ \\
\hline LDL-D & $-.057(.042)$ & $.367(.03)$ & $.21(.053)$ & $.145(.061)$ \\
\hline S-LDL-C & $-.062(.043)$ & $.614(.126)$ & $.261(.062)$ & $-.282(.152)$ \\
\hline S-LDL-L & $-.06(.044)$ & $.584(.118)$ & $.266(.068)$ & $-.251(.145)$ \\
\hline S-LDL-P & $-.033(.047)$ & $.589(.119)$ & $.29(.078)$ & $-.266(.151)$ \\
\hline M-LDL-C & $-.082(.044)$ & $.623(.146)$ & $.203(.054)$ & $-.271(.162)$ \\
\hline M-LDL-CE & $-.074(.043)$ & $.485(.167)$ & $.169(.059)$ & $-.088(.188)$ \\
\hline
\end{tabular}




\begin{tabular}{|c|c|c|c|c|}
\hline M-LDL-L & $-.071(.044)$ & $.444(.171)$ & $.19(.063)$ & $-.069(.191)$ \\
\hline M-LDL-P & $-.054(.044)$ & $.539(.153)$ & $.213(.063)$ & $-.179(.174)$ \\
\hline M-LDL-PL & $-.081(.045)$ & $.747(.134)$ & $.232(.062)$ & $-.407(.162)$ \\
\hline L-LDL-C & $-.071(.049)$ & $.437(.242)$ & $.167(.054)$ & $-.059(.261)$ \\
\hline L-LDL-CE & $-.07(.048)$ & $.277(.301)$ & $.149(.065)$ & $.116(.321)$ \\
\hline L-LDL-FC & $-.112(.057)$ & $.184(.304)$ & $.163(.053)$ & $.223(.315)$ \\
\hline L-LDL-L & $-.075(.049)$ & $.229(.26)$ & $.146(.068)$ & $.167(.273)$ \\
\hline L-LDL-P & $-.083(.046)$ & $.33(.2)$ & $.128(.064)$ & $.084(.213)$ \\
\hline L-LDL-PL & $-.101(.046)$ & $.446(.177)$ & $.155(.057)$ & $-.036(.195)$ \\
\hline IDL-C & $-.108(.057)$ & $.231(.215)$ & $.128(.064)$ & $.192(.229)$ \\
\hline IDL-FC & $-.107(.05)$ & $.23(.147)$ & $.123(.056)$ & $.19(.156)$ \\
\hline IDL-L & $-.1(.05)$ & $.274(.161)$ & $.123(.069)$ & $.148(.175)$ \\
\hline IDL-P & $-.101(.047)$ & $.269(.134)$ & $.109(.071)$ & $.153(.148)$ \\
\hline IDL-PL & $-.076(.048)$ & $.25(.162)$ & $.134(.071)$ & $.153(.18)$ \\
\hline IDL-TG & $-.083(.046)$ & $.314(.069)$ & $.103(.089)$ & $.11(.103)$ \\
\hline \multicolumn{5}{|l|}{ HDL traits } \\
\hline ApoA1 & $.345(.25)$ & $.544(.081)$ & $.334(.109)$ & $-.481(.271)$ \\
\hline HDL-D & $-.442(.124)$ & $.421(.033)$ & $.111(.055)$ & $.333(.114)$ \\
\hline S-HDL-L & $-.117(.046)$ & $.488(.044)$ & $.189(.054)$ & $-.312(.106)$ \\
\hline S-HDL-P & $-.112(.046)$ & $.453(.035)$ & $.225(.056)$ & $-.331(.095)$ \\
\hline S-HDL-TG & $.002(.145)$ & $.314(.156)$ & $-.007(.469)$ & $.253(.637)$ \\
\hline M-HDL-C & $.179(.097)$ & $.36(.038)$ & $.147(.054)$ & $-.508(.165)$ \\
\hline M-HDL-CE & $.167(.087)$ & $.319(.036)$ & $.166(.055)$ & $-.487(.157)$ \\
\hline M-HDL-FC & $.339(.141)$ & $.436(.04)$ & $.247(.059)$ & $-.649(.225)$ \\
\hline M-HDL-L & $.27(.108)$ & $.362(.032)$ & $.299(.063)$ & $-.606(.188)$ \\
\hline M-HDL-P & $.302(.112)$ & $.386(.033)$ & $.371(.075)$ & $-.694(.204)$ \\
\hline M-HDL-PL & $.311(.117)$ & $.402(.033)$ & $.333(.07)$ & $-.632(.191)$ \\
\hline L-HDL-C & $-.589(.211)$ & $.469(.039)$ & $.146(.055)$ & $.516(.213)$ \\
\hline L-HDL-CE & $-.602(.239)$ & $.477(.042)$ & $.137(.056)$ & $.519(.23)$ \\
\hline L-HDL-FC & $-.573(.177)$ & $.437(.034)$ & $.171(.054)$ & $.518(.181)$ \\
\hline L-HDL-L & $-.556(.193)$ & $.437(.034)$ & $.142(.055)$ & $.457(.189)$ \\
\hline L-HDL-P & $-.515(.198)$ & $.417(.03)$ & $.133(.056)$ & $.422(.191)$ \\
\hline L-HDL-PL & $-.53(.201)$ & $.415(.034)$ & $.152(.055)$ & $.443(.202)$ \\
\hline XL-HDL-C & $-.447(.182)$ & $.342(.036)$ & $.071(.079)$ & $.474(.223)$ \\
\hline XL-HDL-CE & $-.425(.146)$ & $.366(.038)$ & $.051(.069)$ & $.426(.177)$ \\
\hline XL-HDL-FC & $-.459(.147)$ & $.377(.031)$ & $.097(.062)$ & $.433(.16)$ \\
\hline XL-HDL-L & $-.405(.146)$ & $.364(.031)$ & $.077(.068)$ & $.358(.154)$ \\
\hline XL-HDL-P & $-.451(.134)$ & $.374(.03)$ & $.078(.064)$ & $.41(.139)$ \\
\hline XL-HDL-PL & $-.422(.119)$ & $.412(.033)$ & $.115(.055)$ & $.343(.118)$ \\
\hline XL-HDL-TG & $-.186(.073)$ & $.336(.035)$ & $.045(.086)$ & $.147(.074)$ \\
\hline
\end{tabular}


bioRxiv preprint doi: https://doi.org/10.1101/691089; this version posted February 6, 2021. The copyright holder for this preprint (which was not certified by peer review) is the author/funder, who has granted bioRxiv a license to display the preprint in perpetuity. It is made available under aCC-BY-NC-ND 4.0 International license.

Appendix 3-Table 6: Multivariable Mendelian randomization results (adjusted for ApoA1, ApoB, and TG).

\begin{tabular}{|c|c|c|c|c|}
\hline Trait & ApoA1 & ApoB & TG & Subfraction \\
\hline \multicolumn{5}{|l|}{ VLDL traits } \\
\hline VLDL-D & $-.227(.067)$ & $.545(.092)$ & $.208(.139)$ & $-.32(.112)$ \\
\hline XS-VLDL-L & $-.123(.063)$ & $.53(.163)$ & $-.121(.085)$ & $.084(.141)$ \\
\hline XS-VLDL-P & $-.121(.064)$ & $.553(.17)$ & $-.123(.088)$ & $.061(.158)$ \\
\hline XS-VLDL-PL & $-.147(.066)$ & $.273(.138)$ & $.028(.05)$ & $.253(.135)$ \\
\hline XS-VLDL-TG & $-.102(.06)$ & $.762(.168)$ & $.069(.055)$ & $-.248(.15)$ \\
\hline S-VLDL-C & $-.384(.141)$ & $1.426(.354)$ & $.606(.351)$ & $-1.265(.568)$ \\
\hline S-VLDL-FC & $-.188(.077)$ & $1.001(.235)$ & $.081(.053)$ & $-.489(.213)$ \\
\hline S-VLDL-L & $-.46(.146)$ & $1.776(.417)$ & $.7(.316)$ & $-1.629(.586)$ \\
\hline S-VLDL-P & $-.494(.159)$ & $1.677(.386)$ & $.825(.372)$ & $-1.644(.606)$ \\
\hline S-VLDL-PL & $-.262(.097)$ & $1.41(.343)$ & $.532(.261)$ & $-1.213(.478)$ \\
\hline S-VLDL-TG & $-.18(.069)$ & $.792(.121)$ & $.078(.051)$ & $-.301(.108)$ \\
\hline M-VLDL-C & $-.157(.062)$ & $.867(.132)$ & $.085(.051)$ & $-.373(.118)$ \\
\hline M-VLDL-CE & $-.221(.069)$ & $1.224(.223)$ & $.47(.21)$ & $-.995(.338)$ \\
\hline M-VLDL-FC & $-.222(.074)$ & $.902(.133)$ & $.482(.251)$ & $-.799(.311)$ \\
\hline M-VLDL-L & $-.174(.065)$ & $.76(.104)$ & $.073(.05)$ & $-.298(.098)$ \\
\hline M-VLDL-P & $-.181(.065)$ & $.764(.1)$ & $.077(.051)$ & $-.312(.096)$ \\
\hline M-VLDL-PL & $-.159(.065)$ & $.776(.116)$ & $.08(.051)$ & $-.297(.106)$ \\
\hline M-VLDL-TG & $-.263(.106)$ & $.724(.094)$ & $.547(.406)$ & $-.806(.455)$ \\
\hline L-VLDL-C & $-.218(.084)$ & $.732(.101)$ & $.352(.278)$ & $-.609(.337)$ \\
\hline L-VLDL-CE & $-.293(.079)$ & $.781(.096)$ & $.405(.189)$ & $-.673(.217)$ \\
\hline L-VLDL-FC & $-.197(.069)$ & $.737(.094)$ & $.365(.25)$ & -.619 (.291) \\
\hline L-VLDL-L & $-.194(.071)$ & $.666(.087)$ & $.289(.234)$ & $-.532(.278)$ \\
\hline L-VLDL-P & $-.184(.061)$ & $.677(.086)$ & $.415(.217)$ & -.617 (.229) \\
\hline L-VLDL-PL & $-.155(.063)$ & $.715(.095)$ & $.075(.051)$ & $-.287(.104)$ \\
\hline L-VLDL-TG & $-.154(.062)$ & $.67(.083)$ & $.073(.05)$ & $-.252(.091)$ \\
\hline XL-VLDL-L & $-.186(.066)$ & $.694(.088)$ & $.263(.19)$ & $-.577(.249)$ \\
\hline XL-VLDL-P & $-.167(.061)$ & $.742(.088)$ & $.075(.05)$ & $-.373(.109)$ \\
\hline XL-VLDL-PL & $-.191(.068)$ & $.712(.092)$ & $.271(.197)$ & $-.583(.268)$ \\
\hline XL-VLDL-TG & $-.195(.068)$ & $.666(.087)$ & $.334(.21)$ & $-.603(.248)$ \\
\hline XXL-VLDL-L & $-.173(.066)$ & $.732(.098)$ & $.088(.052)$ & $-.402(.144)$ \\
\hline XXL-VLDL-P & $-.071(.065)$ & $.705(.097)$ & $.607(.321)$ & $-1.089(.449)$ \\
\hline XXL-VLDL-PL & $-.244(.082)$ & $.666(.091)$ & $.414(.257)$ & $-.814(.344)$ \\
\hline XXL-VLDL-TG & $-.3(.091)$ & $.694(.095)$ & $.627(.306)$ & $-1.075(.402)$ \\
\hline \multicolumn{5}{|l|}{ IDL/LDL traits } \\
\hline LDL-C & $-.119(.062)$ & $.247(.167)$ & $.066(.054)$ & $.319(.182)$ \\
\hline LDL-D & $-.123(.06)$ & $.544(.091)$ & $-.036(.087)$ & $.119(.071)$ \\
\hline S-LDL-C & $-.097(.06)$ & $.438(.216)$ & $.044(.051)$ & $.08(.238)$ \\
\hline S-LDL-L & $-.097(.063)$ & $.503(.268)$ & $.043(.051)$ & $-.005(.29)$ \\
\hline S-LDL-P & $-.059(.103)$ & $.932(.597)$ & $-.122(.112)$ & $-.362(.596)$ \\
\hline M-LDL-C & $-.099(.065)$ & $.78(1.034)$ & $-.172(.425)$ & $-.169(.909)$ \\
\hline M-LDL-CE & $-.157(.128)$ & $-.346(2.587)$ & $.195(.855)$ & $.854(2.221)$ \\
\hline M-LDL-L & $-.123(.095)$ & $.247(1.479)$ & $-.001(.445)$ & $.32(1.293)$ \\
\hline
\end{tabular}




\begin{tabular}{|c|c|c|c|c|}
\hline M-LDL-P & $-.134(.07)$ & $.13(.286)$ & $.053(.052)$ & $.432(.31)$ \\
\hline M-LDL-PL & $-.075(.077)$ & $1.165(.868)$ & $-.248(.253)$ & $-.566(.839)$ \\
\hline L-LDL-C & $-.855(1.68)$ & $-5.337(13.402)$ & $2.405(5.735)$ & $5.257(11.72)$ \\
\hline L-LDL-CE & $-.151(.065)$ & $.129(.193)$ & $.061(.052)$ & $.461(.213)$ \\
\hline L-LDL-FC & $-.397(.219)$ & $-1.139(1.395)$ & $.786(.711)$ & $1.531(1.189)$ \\
\hline L-LDL-L & $-.265(.148)$ & $-.854(1.42)$ & $.41(.51)$ & $1.266(1.188)$ \\
\hline L-LDL-P & $-.258(.153)$ & $-.607(1.225)$ & $.276(.402)$ & $1.064(1.029)$ \\
\hline L-LDL-PL & $-.312(.187)$ & $-.741(1.411)$ & $.39(.518)$ & $1.227(1.245)$ \\
\hline IDL-C & $-.3(.123)$ & $-.334(.616)$ & $.276(.254)$ & $.769(.501)$ \\
\hline IDL-FC & $-.199(.069)$ & $.247(.118)$ & $.044(.049)$ & $.33(.127)$ \\
\hline IDL-L & $-.215(.089)$ & $.021(.409)$ & $.101(.15)$ & $.444(.328)$ \\
\hline IDL-P & $-.175(.075)$ & $.214(.172)$ & $.04(.051)$ & $.292(.173)$ \\
\hline IDL-PL & $-.183(.07)$ & $.159(.172)$ & $.031(.049)$ & $.406(.184)$ \\
\hline IDL-TG & $-.143(.075)$ & $.565(.146)$ & $-.119(.087)$ & $.047(.135)$ \\
\hline \multicolumn{5}{|l|}{ HDL traits } \\
\hline HDL-C & $-1.513(1.109)$ & $.982(.314)$ & $.27(.291)$ & $1.446(1.112)$ \\
\hline HDL-D & $-.457(.138)$ & $.613(.073)$ & $.056(.049)$ & $.296(.1)$ \\
\hline S-HDL-L & $-.128(.059)$ & $.524(.062)$ & $.067(.05)$ & $-.224(.087)$ \\
\hline S-HDL-P & $-.132(.059)$ & $.531(.059)$ & $.071(.05)$ & $-.24(.083)$ \\
\hline S-HDL-TG & $-.11(.113)$ & $.595(.221)$ & $-.057(.297)$ & $-.044(.466)$ \\
\hline M-HDL-C & $.091(.084)$ & $.459(.101)$ & $-.1(.083)$ & $-.442(.143)$ \\
\hline M-HDL-CE & $.09(.078)$ & $.291(.083)$ & $.082(.05)$ & $-.413(.137)$ \\
\hline M-HDL-FC & $.148(.11)$ & $.378(.063)$ & $.066(.049)$ & $-.408(.166)$ \\
\hline M-HDL-L & $.133(.091)$ & $.491(.097)$ & $-.029(.086)$ & $-.485(.155)$ \\
\hline M-HDL-P & $.129(.097)$ & $.501(.097)$ & $-.004(.09)$ & $-.472(.166)$ \\
\hline M-HDL-PL & $.162(.107)$ & $.519(.096)$ & $-.037(.087)$ & $-.486(.171)$ \\
\hline L-HDL-C & $-.724(.232)$ & $.856(.132)$ & $.032(.093)$ & $.575(.204)$ \\
\hline L-HDL-CE & $-.761(.236)$ & $.899(.145)$ & $.004(.084)$ & $.61(.206)$ \\
\hline L-HDL-FC & $-.749(.174)$ & $.842(.102)$ & $.094(.05)$ & $.59(.148)$ \\
\hline L-HDL-L & $-.717(.217)$ & $.815(.12)$ & $.023(.089)$ & $.541(.184)$ \\
\hline L-HDL-P & $-.653(.191)$ & $.749(.104)$ & $.057(.049)$ & $.476(.155)$ \\
\hline L-HDL-PL & $-.679(.201)$ & $.774(.109)$ & $.05(.049)$ & $.51(.169)$ \\
\hline XL-HDL-C & $-.639(.194)$ & $.692(.095)$ & $-.058(.086)$ & $.565(.196)$ \\
\hline XL-HDL-CE & $-.576(.2)$ & $.667(.096)$ & $-.077(.086)$ & $.511(.206)$ \\
\hline XL-HDL-FC & $-.734(.174)$ & $.674(.073)$ & $.094(.052)$ & $.609(.159)$ \\
\hline XL-HDL-L & $-.652(.168)$ & $.733(.097)$ & $-.06(.084)$ & $.481(.141)$ \\
\hline XL-HDL-P & $-.52(.147)$ & $.691(.094)$ & $-.075(.084)$ & $.39(.135)$ \\
\hline XL-HDL-PL & $-.652(.151)$ & $.687(.076)$ & $.079(.051)$ & $.466(.12)$ \\
\hline XL-HDL-TG & $-.281(.111)$ & $.539(.09)$ & $-.152(.092)$ & $.165(.086)$ \\
\hline
\end{tabular}




\section{Appendix 3.4 Q-statistics for multivariable Mendelian randomization}

Appendix 3-Table 7: Modified Cochran's Q-statistics (p-values) for the multivariable Mendelian randomization analyses (adjusted for HDL-C, LDL-C, and TG). DF is short for degrees of freedom.

\begin{tabular}{|c|c|c|c|c|c|}
\hline Trait & $\mathrm{DF}$ & HDL-C & LDL-C & TG & Subfraction \\
\hline \multicolumn{6}{|l|}{ VLDL traits } \\
\hline VLDL-D & 432 & $7640.8(0)$ & $1918.9(7.9 \mathrm{e}-186)$ & $877.6(1.4 \mathrm{e}-32)$ & $840.2(1.6 \mathrm{e}-28)$ \\
\hline XS-VLDL-L & 436 & $7983.9(0)$ & $1104.9(1.1 \mathrm{e}-59)$ & $1935.8(2.2 \mathrm{e}-187)$ & $926(1.9 \mathrm{e}-37)$ \\
\hline XS-VLDL-P & 436 & $7927.8(0)$ & $1066.6(1.1 \mathrm{e}-54)$ & $1814(4.8 \mathrm{e}-167)$ & $893.6(9.6 \mathrm{e}-34)$ \\
\hline XS-VLDL-PL & 435 & $8291.5(0)$ & $968.1(1.4 \mathrm{e}-42)$ & $2771.5(0)$ & $849.8(4.3 \mathrm{e}-29)$ \\
\hline XS-VLDL-TG & 431 & $7549.8(0)$ & $894.4(1.3 \mathrm{e}-34)$ & $739.5(1.3 \mathrm{e}-18)$ & $682.5(1.2 \mathrm{e}-13)$ \\
\hline S-VLDL-C & 429 & $8598.1(0)$ & $652.6(1.7 \mathrm{e}-11)$ & $1220.7(4.6 \mathrm{e}-77)$ & $541.3(0.00018)$ \\
\hline S-VLDL-FC & 434 & $7861.2(0)$ & $576(5.4 \mathrm{e}-06)$ & $519.4(0.003)$ & $507.9(0.0082)$ \\
\hline S-VLDL-L & 438 & $7105.3(0)$ & $626(8.5 \mathrm{e}-09)$ & $525.2(0.0026)$ & $514.3(0.0069)$ \\
\hline S-VLDL-P & 438 & $6686.5(0)$ & $616.5(3.6 \mathrm{e}-08)$ & $515.6(0.0061)$ & $507.3(0.012)$ \\
\hline S-VLDL-PL & 437 & $7589.1(0)$ & $702.8(1 \mathrm{e}-14)$ & $591.5(1.1 \mathrm{e}-06)$ & $555.1(0.00011)$ \\
\hline S-VLDL-TG & 437 & $7658.7(0)$ & $612.7(5.3 \mathrm{e}-08)$ & $498.9(0.021)$ & $494.5(0.03)$ \\
\hline M-VLDL-C & 432 & $9167.8(0)$ & $740.8(1.3 \mathrm{e}-18)$ & $558.9(3.5 \mathrm{e}-05)$ & $551.5(8.3 \mathrm{e}-05)$ \\
\hline M-VLDL-CE & 432 & $8055.2(0)$ & $705.9(1.6 \mathrm{e}-15)$ & $556.6(4.6 \mathrm{e}-05)$ & $539.7(0.00031)$ \\
\hline M-VLDL-FC & 436 & $8272.8(0)$ & $814.8(2.7 \mathrm{e}-25)$ & $528.3(0.0016)$ & $519.1(0.0037)$ \\
\hline M-VLDL-L & 429 & $7109.2(0)$ & $1269.2(5.5 \mathrm{e}-84)$ & $532.6(0.00047)$ & $515.9(0.0025)$ \\
\hline M-VLDL-P & 436 & $8260.7(0)$ & $2059.5(2.1 \mathrm{e}-208)$ & $527.5(0.0017)$ & $516.8(0.0046)$ \\
\hline M-VLDL-PL & 435 & $6849.2(0)$ & $599.6(2.6 \mathrm{e}-07)$ & $496.8(0.021)$ & $493.5(0.027)$ \\
\hline M-VLDL-TG & 436 & $6123.7(0)$ & $9854.8(0)$ & $532.3(0.0011)$ & $521(0.0031)$ \\
\hline L-VLDL-C & 435 & $8617.2(0)$ & $8966(0)$ & $654.7(4.3 \mathrm{e}-11)$ & $561.5(3.9 \mathrm{e}-05)$ \\
\hline L-VLDL-CE & 434 & $6636.6(0)$ & $11134(0)$ & $581.6(2.6 \mathrm{e}-06)$ & $539.5(0.00041)$ \\
\hline L-VLDL-FC & 431 & $7779.6(0)$ & $6691(0)$ & $595.1(2.5 \mathrm{e}-07)$ & $562.7(1.9 \mathrm{e}-05)$ \\
\hline L-VLDL-L & 434 & $8104.9(0)$ & $5191.4(0)$ & $560.3(3.9 \mathrm{e}-05)$ & $548.6(0.00015)$ \\
\hline L-VLDL-P & 435 & $2308(5.1 \mathrm{e}-252)$ & $10360.3(0)$ & $545.4(0.00024)$ & $537.9(0.00054)$ \\
\hline L-VLDL-PL & 430 & $8155.4(0)$ & $1310.8(8.6 \mathrm{e}-90)$ & $491.8(0.021)$ & $489.7(0.024)$ \\
\hline L-VLDL-TG & 438 & $8581.8(0)$ & $4800.1(0)$ & $569.1(2.3 \mathrm{e}-05)$ & $559.2(7.5 \mathrm{e}-05)$ \\
\hline XL-VLDL-L & 437 & $8686.8(0)$ & $8322.2(0)$ & $674.7(1.9 \mathrm{e}-12)$ & $620.2(1.7 \mathrm{e}-08)$ \\
\hline XL-VLDL-P & 431 & $8550.2(0)$ & $2459.4(2 \mathrm{e}-280)$ & $608.3(3.6 \mathrm{e}-08)$ & $588.6(6.3 \mathrm{e}-07)$ \\
\hline XL-VLDL-PL & 431 & $7478.2(0)$ & $5042.5(0)$ & $613.3(1.7 \mathrm{e}-08)$ & $591.6(4.1 \mathrm{e}-07)$ \\
\hline XL-VLDL-TG & 433 & $8237.3(0)$ & $9628.9(0)$ & $651.8(4.6 \mathrm{e}-11)$ & $618.3(1.1 \mathrm{e}-08)$ \\
\hline XXL-VLDL-L & 439 & $8476.2(0)$ & $10436.4(0)$ & $652.9(1.3 \mathrm{e}-10)$ & $570.7(2.2 \mathrm{e}-05)$ \\
\hline XXL-VLDL-P & 437 & $1291.3(2.8 \mathrm{e}-85)$ & $9987.4(0)$ & $540.3(0.00053)$ & $529.5(0.0016)$ \\
\hline XXL-VLDL-PL & 436 & $9631.8(0)$ & $11287.1(0)$ & $641.6(4.8 \mathrm{e}-10)$ & $595.5(5.3 \mathrm{e}-07)$ \\
\hline XXL-VLDL-TG & 429 & $7809.4(0)$ & $9476.4(0)$ & $595.6(1.7 \mathrm{e}-07)$ & $564(1.2 \mathrm{e}-05)$ \\
\hline \multicolumn{6}{|l|}{ LDL/IDL traits } \\
\hline ApoB & 435 & $9220.8(0)$ & $550.1(0.00014)$ & $1809.7(1.2 \mathrm{e}-166)$ & $535.1(0.00072)$ \\
\hline LDL-D & 429 & $2909.2(0)$ & $3918.8(0)$ & $2706(0)$ & $1426.1(2.9 \mathrm{e}-107)$ \\
\hline S-LDL-C & 431 & $8189.7(0)$ & $569.8(7.8 \mathrm{e}-06)$ & $4880.9(0)$ & $564.1(1.6 \mathrm{e}-05)$ \\
\hline S-LDL-L & 435 & $8403.8(0)$ & $574.4(7.8 \mathrm{e}-06)$ & $3931.2(0)$ & $564.3(2.7 \mathrm{e}-05)$ \\
\hline S-LDL-P & 431 & $7371.4(0)$ & $547.1(0.00012)$ & $3144.7(0)$ & $537.9(0.00034)$ \\
\hline M-LDL-C & 430 & $9723.7(0)$ & $570.9(5.8 \mathrm{e}-06)$ & $6568.6(0)$ & $562.9(1.6 \mathrm{e}-05)$ \\
\hline
\end{tabular}




\begin{tabular}{|c|c|c|c|c|c|}
\hline Trait & $\mathrm{DF}$ & ApoA1 & ApoB & TG & Subfraction \\
\hline M-LDL-CE & 432 & $8442.1(0)$ & $558.3(3.8 \mathrm{e}-05)$ & $5773.6(0)$ & $549.1(0.00011)$ \\
\hline M-LDL-L & 430 & $8801.7(0)$ & $555.4(4 \mathrm{e}-05)$ & $5176.1(0)$ & $548.2(9.5 \mathrm{e}-05)$ \\
\hline M-LDL-P & 429 & $8798.9(0)$ & $541.6(0.00018)$ & $5049.7(0)$ & $535.2(0.00035)$ \\
\hline M-LDL-PL & 436 & $7981.7(0)$ & $573.9(9.6 \mathrm{e}-06)$ & $4304.8(0)$ & $558.9(6 \mathrm{e}-05)$ \\
\hline L-LDL-C & 432 & $8865.2(0)$ & $567.7(1.2 \mathrm{e}-05)$ & $6179.8(0)$ & $567(1.3 \mathrm{e}-05)$ \\
\hline L-LDL-CE & 433 & $8464.3(0)$ & $558.7(4.1 \mathrm{e}-05)$ & $5731.3(0)$ & $555.6(5.9 \mathrm{e}-05)$ \\
\hline L-LDL-FC & 431 & $7481.1(0)$ & $580.6(1.9 \mathrm{e}-06)$ & $6760.8(0)$ & $580.2(2 \mathrm{e}-06)$ \\
\hline L-LDL-L & 433 & $8486.8(0)$ & $604.5(8.9 \mathrm{e}-08)$ & $5755.8(0)$ & $601.8(1.3 \mathrm{e}-07)$ \\
\hline L-LDL-P & 434 & $8310.7(0)$ & $592.1(6.3 \mathrm{e}-07)$ & $5553.3(0)$ & $584.9(1.7 \mathrm{e}-06)$ \\
\hline L-LDL-PL & 435 & $8341.4(0)$ & $588.5(1.2 \mathrm{e}-06)$ & $5327.8(0)$ & $577.4(5.3 \mathrm{e}-06)$ \\
\hline IDL-C & 434 & $7873.9(0)$ & $645.5(1.7 \mathrm{e}-10)$ & $6336(0)$ & $642.1(2.9 \mathrm{e}-10)$ \\
\hline IDL-FC & 432 & $8036(0)$ & $729.5(1.4 \mathrm{e}-17)$ & $6630.5(0)$ & $725.6(3 \mathrm{e}-17)$ \\
\hline IDL-L & 434 & $7869.8(0)$ & $694.5(2.4 \mathrm{e}-14)$ & $5198.3(0)$ & $689(7 \mathrm{e}-14)$ \\
\hline IDL-P & 436 & $9660.5(0)$ & $736.7(9 \mathrm{e}-18)$ & $5002(0)$ & $726.6(7.1 \mathrm{e}-17)$ \\
\hline IDL-PL & 431 & $8432.6(0)$ & $680.6(1.7 \mathrm{e}-13)$ & $5023(0)$ & $677.4(3 \mathrm{e}-13)$ \\
\hline IDL-TG & 436 & $7741.2(0)$ & $1077.5(4.2 \mathrm{e}-56)$ & $1992.9(4.9 \mathrm{e}-197)$ & $931.6(4.4 \mathrm{e}-38)$ \\
\hline \multicolumn{6}{|l|}{ HDL traits } \\
\hline ApoA1 & 434 & $494.1(0.024)$ & $511.5(0.006)$ & $932.1(1.8 \mathrm{e}-38)$ & $492(0.028)$ \\
\hline HDL-D & 438 & $783.5(6.6 \mathrm{e}-22)$ & $8500(0)$ & $5713.2(0)$ & $860.1(9.4 \mathrm{e}-30)$ \\
\hline S-HDL-L & 438 & $3067.3(0)$ & $4414.6(0)$ & $3763.2(0)$ & $882.2(3.7 \mathrm{e}-32)$ \\
\hline S-HDL-P & 438 & $2592.4(1.1 \mathrm{e}-301)$ & $7652.1(0)$ & $3097.3(0)$ & $951.1(4.9 \mathrm{e}-40)$ \\
\hline S-HDL-TG & 425 & $896.9(6.9 \mathrm{e}-36)$ & $641.3(5.2 \mathrm{e}-11)$ & $540.1(0.00013)$ & $523(8 \mathrm{e}-04)$ \\
\hline M-HDL-C & 437 & $957.6(5.5 \mathrm{e}-41)$ & $10172.4(0)$ & $4875.5(0)$ & $628.3(4.9 \mathrm{e}-09)$ \\
\hline M-HDL-CE & 434 & $955.3(3.2 \mathrm{e}-41)$ & $1383.1(1.7 \mathrm{e}-99)$ & $4355.4(0)$ & $648.3(1 \mathrm{e}-10)$ \\
\hline M-HDL-FC & 432 & $759.4(2.4 \mathrm{e}-20)$ & $2989.1(0)$ & $3512.2(0)$ & $538.2(0.00037)$ \\
\hline M-HDL-L & 435 & $914.2(3 \mathrm{e}-36)$ & $11535.3(0)$ & $2327.7(1.7 \mathrm{e}-255)$ & $570.3(1.3 \mathrm{e}-05)$ \\
\hline M-HDL-P & 434 & $997.6(2.3 \mathrm{e}-46)$ & $10709.6(0)$ & $1942.9(3.2 \mathrm{e}-189)$ & $561.3(3.4 \mathrm{e}-05)$ \\
\hline M-HDL-PL & 434 & $977.8(6.3 \mathrm{e}-44)$ & $9439.9(0)$ & $2566(1.8 \mathrm{e}-298)$ & $581.3(2.7 \mathrm{e}-06)$ \\
\hline L-HDL-C & 434 & $580(3.2 \mathrm{e}-06)$ & $1257.1(4.4 \mathrm{e}-81)$ & $4502.7(0)$ & $604.3(1.1 \mathrm{e}-07)$ \\
\hline L-HDL-CE & 434 & $549(0.00014)$ & $930.2(3 \mathrm{e}-38)$ & $5517.2(0)$ & $557.2(5.6 \mathrm{e}-05)$ \\
\hline L-HDL-FC & 441 & $627.6(1.2 \mathrm{e}-08)$ & $8415.3(0)$ & $3594(0)$ & $658.4(7.9 \mathrm{e}-11)$ \\
\hline L-HDL-L & 434 & $603.6(1.2 \mathrm{e}-07)$ & $6743.8(0)$ & $5314.7(0)$ & $623.7(5.7 \mathrm{e}-09)$ \\
\hline L-HDL-P & 432 & $601.1(1.2 \mathrm{e}-07)$ & $7769.3(0)$ & $6024.6(0)$ & $633.2(8.6 \mathrm{e}-10)$ \\
\hline L-HDL-PL & 434 & $584.5(1.8 \mathrm{e}-06)$ & $9935.5(0)$ & $3544.3(0)$ & $611.3(3.8 \mathrm{e}-08)$ \\
\hline XL-HDL-C & 430 & $732.9(3.9 \mathrm{e}-18)$ & $10426.6(0)$ & $2077.7(1.4 \mathrm{e}-213)$ & $686.9(4 \mathrm{e}-14)$ \\
\hline XL-HDL-CE & 430 & $771.4(9.3 \mathrm{e}-22)$ & $8564.4(0)$ & $2457(2.2 \mathrm{e}-280)$ & $711.4(3.3 \mathrm{e}-16)$ \\
\hline XL-HDL-FC & 432 & $761.8(1.4 \mathrm{e}-20)$ & $11265.2(0)$ & $2549.4(3.1 \mathrm{e}-296)$ & $770.9(1.9 \mathrm{e}-21)$ \\
\hline XL-HDL-L & 429 & $767.6(1.6 \mathrm{e}-21)$ & $11490.7(0)$ & $2355.7(1.2 \mathrm{e}-262)$ & $784.6(3.4 \mathrm{e}-23)$ \\
\hline XL-HDL-P & 433 & $724.9(4.6 \mathrm{e}-17)$ & $11372.5(0)$ & $2539.9(3.9 \mathrm{e}-294)$ & $798.5(4.8 \mathrm{e}-24)$ \\
\hline XL-HDL-PL & 443 & $809.7(7.8 \mathrm{e}-24)$ & $10093.1(0)$ & $5762(0)$ & $895.4(7.5 \mathrm{e}-33)$ \\
\hline XL-HDL-TG & 432 & $1849.1(3.9 \mathrm{e}-174)$ & $2635.9(6.5 \mathrm{e}-312)$ & $2240.8(2.9 \mathrm{e}-241)$ & $1267.8(4.4 \mathrm{e}-83)$ \\
\hline
\end{tabular}


Appendix 3-Table 8: Modified Cochran's Q-statistics (p-values) for the multivariable Mendelian randomization analyses (adjusted for ApoA1, ApoB, and TG). DF is short for degrees of freedom.

\begin{tabular}{|c|c|c|c|c|c|}
\hline Trait & DF & ApoA1 & ApoB & TG & Subfraction \\
\hline \multicolumn{6}{|l|}{ VLDL traits } \\
\hline VLDL-D & 297 & $1194.1(9.1 \mathrm{e}-108)$ & $550(2.4 \mathrm{e}-17)$ & $573.7(8.2 \mathrm{e}-20)$ & $606.7(2.1 \mathrm{e}-23)$ \\
\hline XS-VLDL-L & 295 & $1185.1(6.7 \mathrm{e}-107)$ & $927(2 \mathrm{e}-66)$ & $1151.3(2.2 \mathrm{e}-101)$ & $887.9(1.1 \mathrm{e}-60)$ \\
\hline XS-VLDL-P & 295 & $1194.9(1.7 \mathrm{e}-108)$ & $900(1.9 \mathrm{e}-62)$ & $895.5(8.7 \mathrm{e}-62)$ & $826.7(6.4 \mathrm{e}-52)$ \\
\hline XS-VLDL-PL & 296 & $1148.5(1.2 \mathrm{e}-100)$ & $973.9(3.2 \mathrm{e}-73)$ & $2104.2(1.4 \mathrm{e}-269)$ & $961.4(2.5 \mathrm{e}-71)$ \\
\hline XS-VLDL-TG & 302 & $1263.7(1.1 \mathrm{e}-117)$ & $757.9(4.7 \mathrm{e}-41)$ & $1308.1(4.4 \mathrm{e}-125)$ & $976.5(4.6 \mathrm{e}-72)$ \\
\hline S-VLDL-C & 290 & $988.8(4.4 \mathrm{e}-77)$ & $394(4.5 \mathrm{e}-05)$ & $459.8(7.8 \mathrm{e}-10)$ & $402.6(1.3 \mathrm{e}-05)$ \\
\hline S-VLDL-FC & 296 & $1092(1.4 \mathrm{e}-91)$ & $904(8.6 \mathrm{e}-63)$ & $1238.7(2.1 \mathrm{e}-115)$ & $1010.4(8.1 \mathrm{e}-79)$ \\
\hline S-VLDL-L & 301 & $1107.9(1.1 \mathrm{e}-92)$ & $412.3(2.1 \mathrm{e}-05)$ & $420.8(5.9 \mathrm{e}-06)$ & $384.7(0.00078)$ \\
\hline S-VLDL-P & 301 & $1116.6(4.6 \mathrm{e}-94)$ & $424.8(3.3 \mathrm{e}-06)$ & $401.3(9.4 \mathrm{e}-05)$ & $380.6(0.0013)$ \\
\hline S-VLDL-PL & 299 & $1096(2.3 \mathrm{e}-91)$ & $428.9(1.2 \mathrm{e}-06)$ & $446(7.1 \mathrm{e}-08)$ & $432.1(7.1 \mathrm{e}-07)$ \\
\hline S-VLDL-TG & 300 & $1152.4(4.3 \mathrm{e}-100)$ & $908.5(1.8 \mathrm{e}-62)$ & $1453.4(1.8 \mathrm{e}-150)$ & $1303.1(7.1 \mathrm{e}-125)$ \\
\hline M-VLDL-C & 298 & $1171.2(1 \mathrm{e}-103)$ & $824(7.3 \mathrm{e}-51)$ & $1480(8.9 \mathrm{e}-156)$ & $1212.5(1.8 \mathrm{e}-110)$ \\
\hline M-VLDL-CE & 298 & $1185.4(4.9 \mathrm{e}-106)$ & $564.4(1.1 \mathrm{e}-18)$ & $468.9(9.2 \mathrm{e}-10)$ & $431.6(6.3 \mathrm{e}-07)$ \\
\hline M-VLDL-FC & 298 & $1190.4(7.4 \mathrm{e}-107)$ & $899.8(1.1 \mathrm{e}-61)$ & $415.2(8.1 \mathrm{e}-06)$ & $398.8(8.4 \mathrm{e}-05)$ \\
\hline M-VLDL-L & 298 & $1144.1(2.4 \mathrm{e}-99)$ & $869.8(2.4 \mathrm{e}-57)$ & 1381 (1e-138) & $1237.4(1.4 \mathrm{e}-114)$ \\
\hline M-VLDL-P & 297 & $1121.3(5.7 \mathrm{e}-96)$ & $821.1(1.1 \mathrm{e}-50)$ & $1250.5(4.6 \mathrm{e}-117)$ & $1206.7(8.1 \mathrm{e}-110)$ \\
\hline M-VLDL-PL & 298 & $1149.9(2.8 \mathrm{e}-100)$ & $843.2(1.5 \mathrm{e}-53)$ & $1391.8(1.5 \mathrm{e}-140)$ & $1226.3(9.8 \mathrm{e}-113)$ \\
\hline M-VLDL-TG & 296 & $1187.4(5.8 \mathrm{e}-107)$ & $717.3(5.8 \mathrm{e}-37)$ & $366.3(0.0033)$ & $360.6(0.006)$ \\
\hline L-VLDL-C & 295 & $1196.5(9.1 \mathrm{e}-109)$ & $820(5.6 \mathrm{e}-51)$ & $462.5(1.5 \mathrm{e}-09)$ & $376.9(0.00088)$ \\
\hline L-VLDL-CE & 302 & $1183.1(1.8 \mathrm{e}-104)$ & $844.6(7.4 \mathrm{e}-53)$ & $541.8(7.2 \mathrm{e}-16)$ & $441.7(2.6 \mathrm{e}-07)$ \\
\hline L-VLDL-FC & 295 & $1172.3(8.2 \mathrm{e}-105)$ & $851.6(1.9 \mathrm{e}-55)$ & $460.8(2.1 \mathrm{e}-09)$ & $406.2(1.8 \mathrm{e}-05)$ \\
\hline L-VLDL-L & 295 & $1163.6(2.2 \mathrm{e}-103)$ & $797(8.8 \mathrm{e}-48)$ & $406.5(1.7 \mathrm{e}-05)$ & $391.5(0.00014)$ \\
\hline L-VLDL-P & 293 & $1160.2(2 \mathrm{e}-103)$ & $809.5(5.9 \mathrm{e}-50)$ & $420.2(1.5 \mathrm{e}-06)$ & $407.9(1 \mathrm{e}-05)$ \\
\hline L-VLDL-PL & 296 & $1292(2.6 \mathrm{e}-124)$ & $833.4(1.3 \mathrm{e}-52)$ & $1216.5(9.7 \mathrm{e}-112)$ & $1098.9(1.1 \mathrm{e}-92)$ \\
\hline L-VLDL-TG & 294 & $1150.8(1.3 \mathrm{e}-101)$ & $1213.6(7 \mathrm{e}-112)$ & $1262.6(5.2 \mathrm{e}-120)$ & $1162.8(1.5 \mathrm{e}-103)$ \\
\hline XL-VLDL-L & 294 & $1196(5.4 \mathrm{e}-109)$ & $829.4(1.6 \mathrm{e}-52)$ & $442(4.9 \mathrm{e}-08)$ & $423.6(1.1 \mathrm{e}-06)$ \\
\hline XL-VLDL-P & 294 & $1265.9(1.4 \mathrm{e}-120)$ & $1180.9(1.6 \mathrm{e}-106)$ & $1202.2(5.2 \mathrm{e}-110)$ & $982.1(5.4 \mathrm{e}-75)$ \\
\hline XL-VLDL-PL & 296 & $1199.1(6.9 \mathrm{e}-109)$ & $874.2(1.9 \mathrm{e}-58)$ & $421.2(2.3 \mathrm{e}-06)$ & $405.6(2.3 \mathrm{e}-05)$ \\
\hline XL-VLDL-TG & 296 & $1184.3(1.8 \mathrm{e}-106)$ & $828.6(5.9 \mathrm{e}-52)$ & $430.8(4.9 \mathrm{e}-07)$ & $430.1(5.5 \mathrm{e}-07)$ \\
\hline XXL-VLDL-L & 304 & $1119.2(1.2 \mathrm{e}-93)$ & $1041.9(1.6 \mathrm{e}-81)$ & $900.9(2 \mathrm{e}-60)$ & $699.6(3.2 \mathrm{e}-33)$ \\
\hline XXL-VLDL-P & 303 & $1148(1.7 \mathrm{e}-98)$ & $876.4(4 \mathrm{e}-57)$ & $382.2(0.0013)$ & $366(0.0076)$ \\
\hline XXL-VLDL-PL & 303 & $1203(2.1 \mathrm{e}-107)$ & $775.1(4 \mathrm{e}-43)$ & $438.1(5.8 \mathrm{e}-07)$ & $376.5(0.0025)$ \\
\hline XXL-VLDL-TG & 303 & $1183(3.7 \mathrm{e}-104)$ & $881.8(6.6 \mathrm{e}-58)$ & $393.7(0.00034)$ & $372.7(0.0039)$ \\
\hline \multicolumn{6}{|l|}{ LDL/IDL traits } \\
\hline LDL-C & 293 & $1198.7(9.6 \mathrm{e}-110)$ & $938.8(1.1 \mathrm{e}-68)$ & $1060.2(2.1 \mathrm{e}-87)$ & $917.6(1.5 \mathrm{e}-65)$ \\
\hline LDL-D & 296 & $1325.2(6.7 \mathrm{e}-130)$ & $747.9(5.9 \mathrm{e}-41)$ & $879.1(3.7 \mathrm{e}-59)$ & $1163.5(4.6 \mathrm{e}-103)$ \\
\hline S-LDL-C & 296 & $1195.3(2.9 \mathrm{e}-108)$ & $706(1.6 \mathrm{e}-35)$ & $1426(4.1 \mathrm{e}-147)$ & $686.4(4.8 \mathrm{e}-33)$ \\
\hline S-LDL-L & 296 & $1054.7(1.1 \mathrm{e}-85)$ & $608(1 \mathrm{e}-23)$ & $1519.6(2.2 \mathrm{e}-163)$ & $586.4(2.5 \mathrm{e}-21)$ \\
\hline S-LDL-P & 297 & $852.9(3.6 \mathrm{e}-55)$ & $438.7(1.6 \mathrm{e}-07)$ & $954.7(4.5 \mathrm{e}-70)$ & $440.1(1.3 \mathrm{e}-07)$ \\
\hline M-LDL-C & 296 & $1210.9(8 \mathrm{e}-111)$ & $396.2(8.6 \mathrm{e}-05)$ & $409(1.4 \mathrm{e}-05)$ & $398.9(6 \mathrm{e}-05)$ \\
\hline M-LDL-CE & 295 & $1204.3(4.8 \mathrm{e}-110)$ & $350.8(0.014)$ & $361.7(0.0048)$ & $351.3(0.013)$ \\
\hline M-LDL-L & 296 & $1212(5.3 \mathrm{e}-111)$ & $370(0.0022)$ & $392.3(0.00015)$ & $371.6(0.0019)$ \\
\hline
\end{tabular}




\begin{tabular}{|c|c|c|c|c|c|}
\hline Trait & $\mathrm{DF}$ & ApoA1 & ApoB & TG & Subfraction \\
\hline M-LDL-P & 297 & $1125.4(1.2 \mathrm{e}-96)$ & $623.9(2.3 \mathrm{e}-25)$ & $911.4(1.3 \mathrm{e}-63)$ & $582.4(9.6 \mathrm{e}-21)$ \\
\hline M-LDL-PL & 299 & $1172.5(1.2 \mathrm{e}-103)$ & $399.3(9.1 \mathrm{e}-05)$ & $434.9(4.5 \mathrm{e}-07)$ & $396.2(0.00014)$ \\
\hline L-LDL-C & 300 & $1174.6(1.1 \mathrm{e}-103)$ & $325.5(0.15)$ & $325.5(0.15)$ & $325.5(0.15)$ \\
\hline L-LDL-CE & 299 & $1179.5(9 \mathrm{e}-105)$ & $769.8(3 \mathrm{e}-43)$ & $902.5(7.7 \mathrm{e}-62)$ & $743.8(8.4 \mathrm{e}-40)$ \\
\hline L-LDL-FC & 295 & $1161(5.8 \mathrm{e}-103)$ & $322.4(0.13)$ & $323.2(0.12)$ & $322.3(0.13)$ \\
\hline L-LDL-L & 300 & $1172.3(2.6 \mathrm{e}-103)$ & $336.9(0.07)$ & $349.6(0.026)$ & $340.3(0.055)$ \\
\hline L-LDL-P & 300 & $1185.4(2 \mathrm{e}-105)$ & $352.1(0.021)$ & $378.4(0.0014)$ & $355.4(0.015)$ \\
\hline L-LDL-PL & 296 & $1155.2(9.8 \mathrm{e}-102)$ & $343.2(0.031)$ & $360.1(0.0063)$ & $344.5(0.027)$ \\
\hline IDL-C & 296 & $1181.7(4.9 \mathrm{e}-106)$ & $426.5(9.8 \mathrm{e}-07)$ & $427.6(8.3 \mathrm{e}-07)$ & $427.7(8.1 \mathrm{e}-07)$ \\
\hline IDL-FC & 298 & $1096.5(9.9 \mathrm{e}-92)$ & $986.9(1.1 \mathrm{e}-74)$ & $1075.8(1.9 \mathrm{e}-88)$ & $975.4(6.1 \mathrm{e}-73)$ \\
\hline IDL-L & 296 & $1176.1(4 \mathrm{e}-105)$ & $516.7(3.3 \mathrm{e}-14)$ & $531(1.4 \mathrm{e}-15)$ & $521.4(1.2 \mathrm{e}-14)$ \\
\hline IDL-P & 297 & $1094.8(9.5 \mathrm{e}-92)$ & $910.9(1.5 \mathrm{e}-63)$ & $1103.9(3.5 \mathrm{e}-93)$ & $890.2(1.6 \mathrm{e}-60)$ \\
\hline IDL-PL & 297 & $1107.8(8.3 \mathrm{e}-94)$ & $798.9(1.3 \mathrm{e}-47)$ & $931.6(1.3 \mathrm{e}-66)$ & $785.6(8.6 \mathrm{e}-46)$ \\
\hline IDL-TG & 302 & $1060.8(5.4 \mathrm{e}-85)$ & $1052.1(1.2 \mathrm{e}-83)$ & $1092.6(5.6 \mathrm{e}-90)$ & $1118.3(4.7 \mathrm{e}-94)$ \\
\hline \multicolumn{6}{|l|}{ HDL traits } \\
\hline HDL-C & 298 & $318.7(0.2)$ & $336.3(0.063)$ & $329.1(0.1)$ & $318.6(0.2)$ \\
\hline HDL-D & 300 & $637.4(1.9 \mathrm{e}-26)$ & $1156.6(9.1 \mathrm{e}-101)$ & $2305.2(1.3 \mathrm{e}-305)$ & $1183.8(3.5 \mathrm{e}-105)$ \\
\hline S-HDL-L & 299 & $1597.7(4.8 \mathrm{e}-176)$ & $1222.5(8.2 \mathrm{e}-112)$ & $1916.4(1.5 \mathrm{e}-233)$ & $1057(3.1 \mathrm{e}-85)$ \\
\hline S-HDL-P & 299 & $1666.8(2.5 \mathrm{e}-188)$ & $1249.4(2.9 \mathrm{e}-116)$ & $2146.5(3.4 \mathrm{e}-276)$ & $1103.3(1.6 \mathrm{e}-92)$ \\
\hline S-HDL-TG & 299 & $899(2.5 \mathrm{e}-61)$ & $464.9(2.4 \mathrm{e}-09)$ & $464.5(2.6 \mathrm{e}-09)$ & $457.6(9.2 \mathrm{e}-09)$ \\
\hline M-HDL-C & 299 & $1145.2(3.2 \mathrm{e}-99)$ & $768.2(4.9 \mathrm{e}-43)$ & $951.8(4 \mathrm{e}-69)$ & $786.8(1.5 \mathrm{e}-45)$ \\
\hline M-HDL-CE & 299 & $1201.9(2 \mathrm{e}-108)$ & $1183.9(1.7 \mathrm{e}-105)$ & $2139.7(6.4 \mathrm{e}-275)$ & $843.9(1.9 \mathrm{e}-53)$ \\
\hline M-HDL-FC & 298 & $881.1(5.6 \mathrm{e}-59)$ & $1252(5.5 \mathrm{e}-117)$ & $1989.1(2.4 \mathrm{e}-247)$ & $660.1(1.8 \mathrm{e}-29)$ \\
\hline M-HDL-L & 299 & $1059(1.5 \mathrm{e}-85)$ & $766.4(8.7 \mathrm{e}-43)$ & $920.6(1.7 \mathrm{e}-64)$ & $672.5(8.6 \mathrm{e}-31)$ \\
\hline M-HDL-P & 298 & $990.2(3.5 \mathrm{e}-75)$ & $760.4(3.4 \mathrm{e}-42)$ & $1027.6(6.2 \mathrm{e}-81)$ & $613.7(4.7 \mathrm{e}-24)$ \\
\hline M-HDL-PL & 295 & $929.5(8.3 \mathrm{e}-67)$ & $763.9(2.7 \mathrm{e}-43)$ & $1057.2(2.3 \mathrm{e}-86)$ & $588.3(1.1 \mathrm{e}-21)$ \\
\hline L-HDL-C & 299 & $579.3(4.1 \mathrm{e}-20)$ & $623.2(5.7 \mathrm{e}-25)$ & $639.6(7.3 \mathrm{e}-27)$ & $617.8(2.3 \mathrm{e}-24)$ \\
\hline L-HDL-CE & 299 & $612.2(1 \mathrm{e}-23)$ & $650.7(3.6 \mathrm{e}-28)$ & $690.4(5.5 \mathrm{e}-33)$ & $644(2.2 \mathrm{e}-27)$ \\
\hline L-HDL-FC & 308 & $581.7(4.4 \mathrm{e}-19)$ & $857.5(2.6 \mathrm{e}-53)$ & $1213.3(1.4 \mathrm{e}-107)$ & $915.8(1.3 \mathrm{e}-61)$ \\
\hline L-HDL-L & 299 & $655.9(8.7 \mathrm{e}-29)$ & $747.7(2.6 \mathrm{e}-40)$ & $670.7(1.4 \mathrm{e}-30)$ & $713.2(7.5 \mathrm{e}-36)$ \\
\hline L-HDL-P & 298 & $591.3(1.5 \mathrm{e}-21)$ & $934(9.9 \mathrm{e}-67)$ & $1269.7(6.2 \mathrm{e}-120)$ & $956.8(3.9 \mathrm{e}-70)$ \\
\hline L-HDL-PL & 299 & $580(3.4 \mathrm{e}-20)$ & $863.5(3.3 \mathrm{e}-56)$ & $1262.4(2.1 \mathrm{e}-118)$ & $891.8(2.8 \mathrm{e}-60)$ \\
\hline XL-HDL-C & 298 & $475.3(2.7 \mathrm{e}-10)$ & $734(1 \mathrm{e}-38)$ & $976.1(4.9 \mathrm{e}-73)$ & $554(1.3 \mathrm{e}-17)$ \\
\hline XL-HDL-CE & 299 & $472.9(5.4 \mathrm{e}-10)$ & $736.9(6.7 \mathrm{e}-39)$ & $1117.4(9 \mathrm{e}-95)$ & $517.5(6.5 \mathrm{e}-14)$ \\
\hline XL-HDL-FC & 295 & $527.8(2.1 \mathrm{e}-15)$ & $1182.8(1.6 \mathrm{e}-106)$ & $2169.4(3.1 \mathrm{e}-282)$ & $677.3(4.3 \mathrm{e}-32)$ \\
\hline XL-HDL-L & 298 & $555.2(9.6 \mathrm{e}-18)$ & $701.2(1.6 \mathrm{e}-34)$ & $1014(7.9 \mathrm{e}-79)$ & $775.3(3.4 \mathrm{e}-44)$ \\
\hline XL-HDL-P & 300 & $578.9(6.3 \mathrm{e}-20)$ & $744.5(1.1 \mathrm{e}-39)$ & $1015.5(1.6 \mathrm{e}-78)$ & $751.3(1.4 \mathrm{e}-40)$ \\
\hline XL-HDL-PL & 306 & $604.9(7.8 \mathrm{e}-22)$ & $1153.9(1.4 \mathrm{e}-98)$ & $1899(1.5 \mathrm{e}-227)$ & $909.3(3.7 \mathrm{e}-61)$ \\
\hline XL-HDL-TG & 300 & $702.2(2.8 \mathrm{e}-34)$ & $779.8(2.2 \mathrm{e}-44)$ & $1140.8(3.2 \mathrm{e}-98)$ & $1399.2(3.7 \mathrm{e}-141)$ \\
\hline
\end{tabular}




\section{Appendix 4 Diagnostic plots and the genetic markers}

As mentioned above, RAPS is more robust against invalid instruments than other statistical methods for univariable MR, but it still needs the InSIDE assumption to be approximately satisfied. Zhao et al. 38. described two diagnostic plots RAPS that checks whether there is clear evidence that the InSIDE assumption is violated. Here we report these plots for HDL-C and M-HDL-P in different studies. Notice that a lack of evidence to falsify the InSIDE assumption does not mean that it is true.

\section{Appendix 4.1 S-HDL-P}
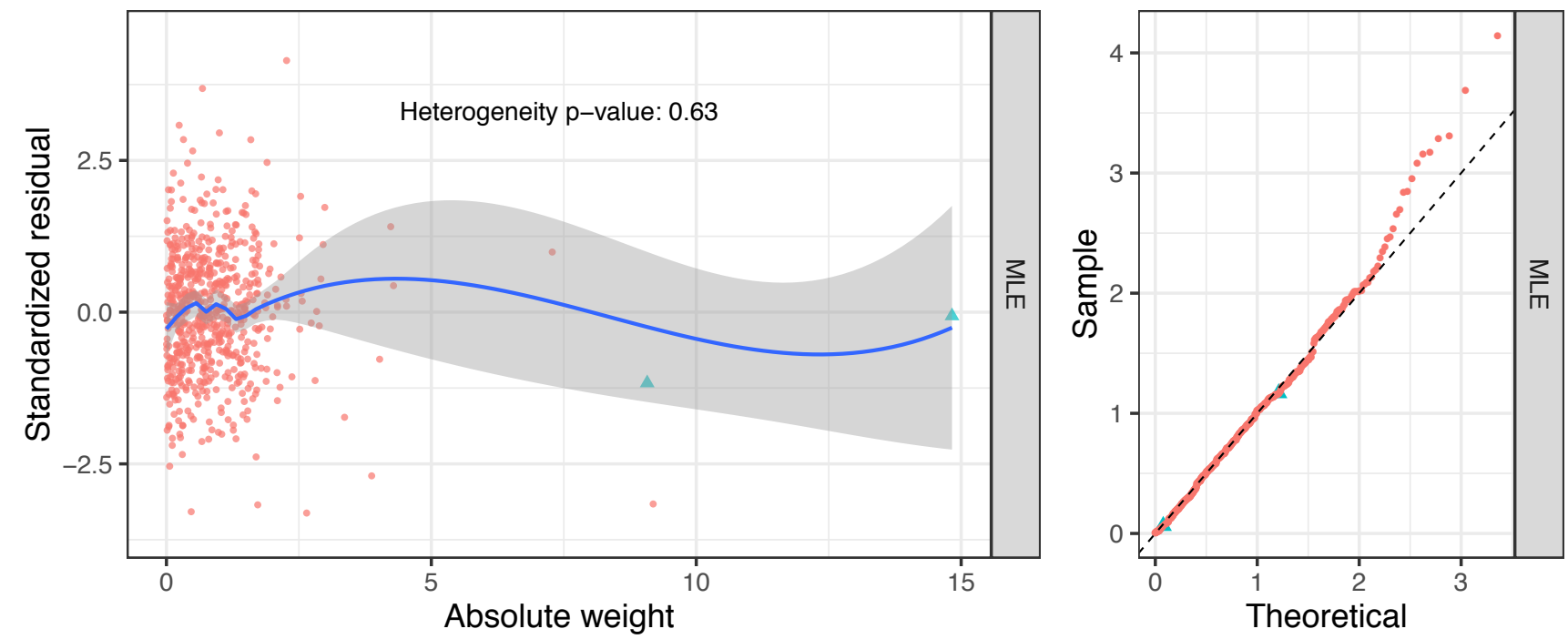

Appendix 4-Figure 1: Diagnostic plots for S-HDL-P (selection: Davis; exposure: Kettunen; outcome: UK Biobank). 


\section{Appendix 4.2 M-HDL-P}
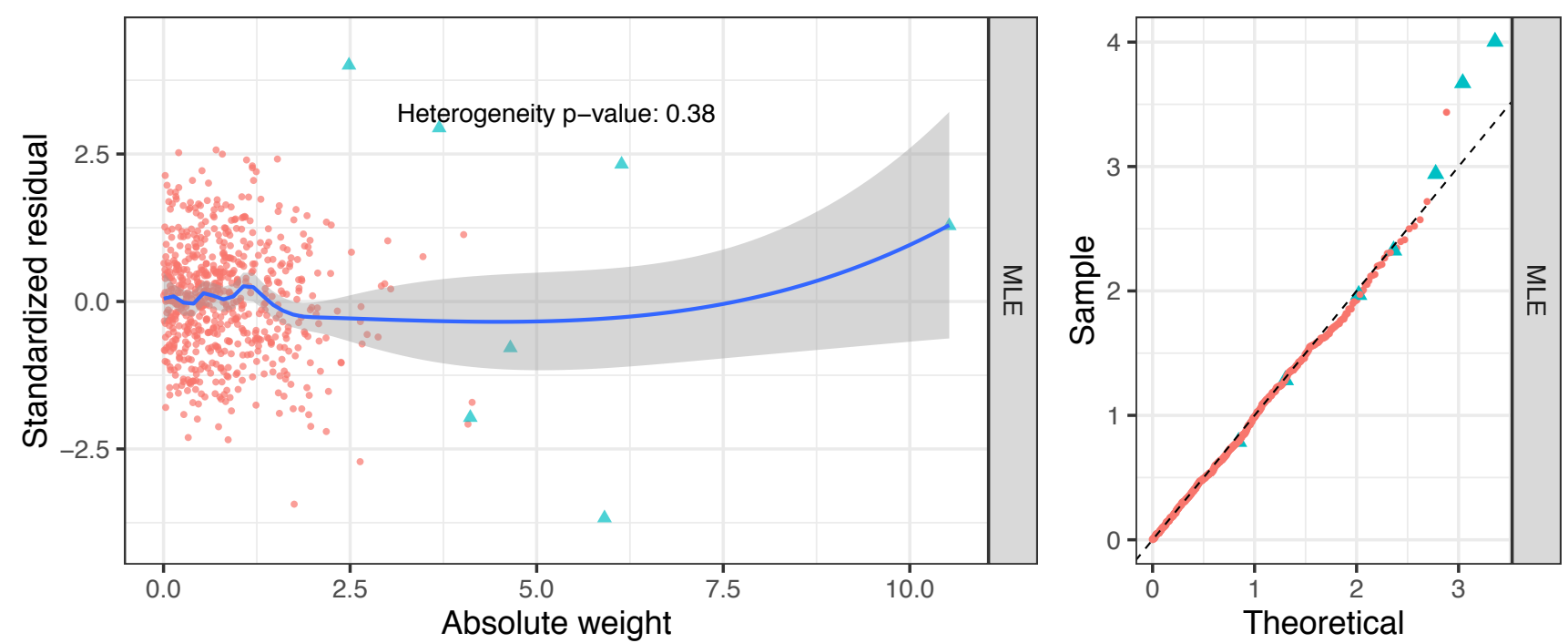

Appendix 4-Figure 2: Diagnostic plots for M-HDL-P (selection: Davis; exposure: Kettunen; outcome: UK Biobank). 


\section{Appendix 4.3 Genetic markers for M-HDL-P and S-HDL-P}

We can further assess the validity of the InSIDE assumption for M-HDL-P and S-HDL-P but examining the associations of their genetic instruments with the traditional lipid risk factors and other subfraction traits. We meta-analyzed the summary results in the two lipidome GWAS (Davis and Kettunen) and obtained SNPs that are associated with S-HDL-P and M-HDL-P (p-value $\leq 5 \times 10^{-8}$; the results are LD-clumped). The next two Tables show some information about these genetic markers and their associations with other traits.

Appendix 4-Figure 3 and 4 shows how adjusting for LDL-C and TG changes the effects of the selected SNPs for S-HDL-P and M-HDL-P on CAD. The adjusted effect on CAD is obtained by original effect on CAD $-0.45 *$ effect on LDL-C $-0.25 *$ effect on TG. After the adjustment, the associations of the genetic variants with CAD generally became closer to the fitted lines that correspond to the estimated effects of S-HDL-P and M-HDL-P.

Appendix 4-Table 1: List of SNPs associated with M-HDL-P.

\begin{tabular}{|c|c|c|c|c|c|c|c|c|c|c|}
\hline $\mathrm{SNP}$ & $\mathrm{Chr}$ & Gene & S-HDL-P & M-HDL-P & L-HDL-P & XL-HDL-P & HDL-C & LDL-C & TG & $\mathrm{CAD}$ \\
\hline rs11208004 & 1 & DOCK7 & $0.039 * *$ & $0.075 * * *$ & 0.015 & -0.002 & 0.015 ** & $0.050 * * *$ & $0.069 * * *$ & 0.012 \\
\hline rs4846913 & 1 & GALNT2 & 0.000 & $0.061 * * *$ & $0.062 * * *$ & 0.023 & $0.055 * * *$ & -0.006 & $-0.044 * * *$ & -0.025 \\
\hline rs2126259 & 8 & LOC157273 & $0.066 * * *$ & $0.082 * * *$ & $0.063 * *$ & 0.025 & $0.075 * * *$ & $0.063^{* * *}$ & -0.016 & -0.004 \\
\hline rs2083637 & 8 & LPL & -0.001 & $0.058 * * *$ & $0.092 * * *$ & $0.053 * *$ & $0.105^{* * *}$ & -0.008 & $-0.108 * * *$ & $-0.047 * *$ \\
\hline rs10468017 & 15 & ALDH1A2/LIPC & $-0.096 * * *$ & $0.060 * * *$ & $0.209 * * *$ & $0.202 * * *$ & $0.118^{* * *}$ & 0.002 & $0.038 * * *$ & 0.013 \\
\hline rs247616 & 16 & CETP & $0.058 * * *$ & $0.121 * * *$ & $0.198 * * *$ & $0.129 * * *$ & $0.243^{* * *}$ & $-0.055 * * *$ & $-0.039 * * *$ & $-0.044 * *$ \\
\hline rs1943973 & 18 & LIPG & 0.022 & $0.108^{* * *}$ & $0.104^{* * *}$ & $0.078 * * *$ & $0.077 * * *$ & $0.024 * *$ & 0.009 & -0.016 \\
\hline rs737337 & 19 & DOCK6 & 0.047 & $0.087 * * *$ & $0.081 * *$ & $0.058^{*}$ & 0.056 *** & 0.007 & -0.011 & -0.038 \\
\hline rs769449 & 19 & APOE & -0.016 & $0.078 * * *$ & $0.071 * * *$ & -0.015 & $0.064 * * *$ & $-0.214 * * *$ & $-0.042 * * *$ & $-0.085 * * *$ \\
\hline rs7679 & 20 & PCIF1/PLTP & $0.188^{* * *}$ & $0.071^{* * *}$ & $-0.129 * * *$ & $-0.152 * * *$ & $-0.059 * * *$ & 0.009 & $0.051 * * *$ & -0.025 \\
\hline
\end{tabular}

Appendix 4-Table 2: List of SNPs associated with S-HDL-P.

\begin{tabular}{|c|c|c|c|c|c|c|c|c|c|c|}
\hline SNP & Chr & Gene & S-HDL-P & M-HDL-P & L-HDL-P & XL-HDL-P & HDL-C & LDL-C & TG & CAD \\
\hline rs780094 & 2 & GCKR & $0.074 * * *$ & $0.034 *$ & $-0.04 * *$ & $-0.034 *$ & -0.011 & $0.021 * *$ & $0.110^{* * *}$ & 0.005 \\
\hline rs10935473 & 3 & ST3GAL6-AS1 & $0.052 * * *$ & 0.014 & -0.029 & $-0.031 *$ & -0.009 & 0.003 & 0.005 & -0.007 \\
\hline rs4936363 & 11 & SIK3 & $0.064 * * *$ & $0.046 * *$ & 0.019 & 0.006 & $0.034^{* *}$ & 0.018 & $0.043^{* * *}$ & 0.022 \\
\hline rs2043085 & 15 & ALDH1A2/LIPC & $0.092 * * *$ & $-0.056 * * *$ & $-0.202 * * *$ & $-0.197 * * *$ & $-0.106 * * *$ & -0.003 & $-0.033 * * *$ & -0.008 \\
\hline rs1800588 & 15 & ALDH1A2/LIPC & $0.106 * * *$ & $-0.050 * *$ & $-0.215 * * *$ & $-0.212 * * *$ & $-0.114 * * *$ & 0.002 & $-0.044 * * *$ & -0.015 \\
\hline rs289714 & 16 & CETP & $0.077 * * *$ & 0.122 *** & $0.162 * * *$ & $0.102 * * *$ & $0.214 * * *$ & $-0.036 * * *$ & $-0.035 * * *$ & -0.012 \\
\hline rs6065904 & 20 & PLTP & $0.171 * * *$ & $0.060 * * *$ & $-0.127 * * *$ & $-0.149 * * *$ & $-0.052 * * *$ & 0.008 & $0.040 * * *$ & -0.022 \\
\hline
\end{tabular}




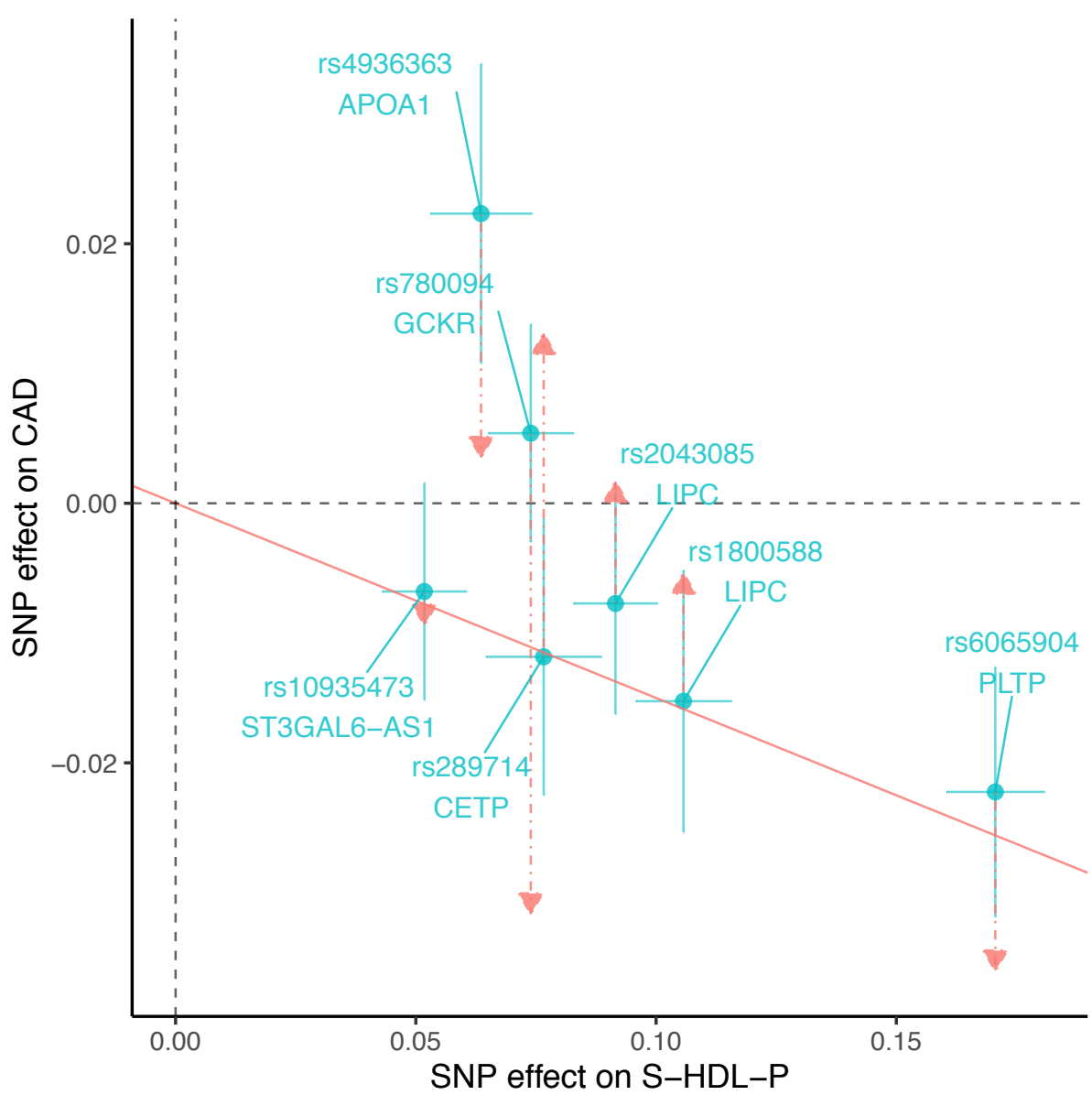

Appendix 4-Figure 3: Scatter-plots for S-HDL-P with the effects on CAD adjusted for LDL-C and TG. Red lines correspond the fitted effects of S-HDL-P in multivariable MR. 


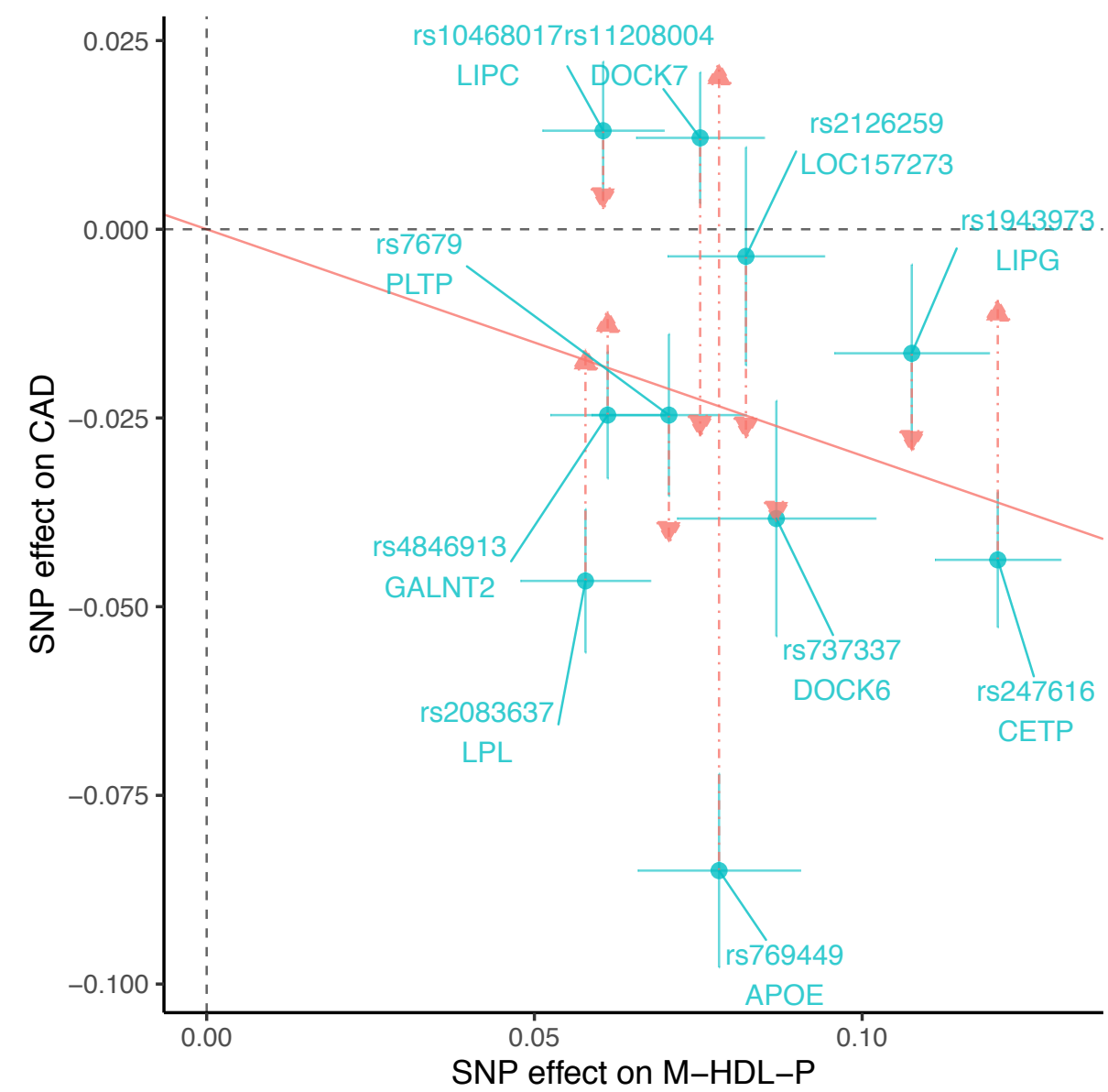

Appendix 4-Figure 4: Scatter-plots for M-HDL-P with the effects on CAD adjusted for LDL-C and TG. Red lines correspond the fitted effects of M-HDL-P in multivariable MR. 


\section{Appendix 4.4 Gene expression}

Appendix 4-Table 3: Tissue-specific gene expressions associated with the 4 discovered genetic markers in the gTEX project.

\begin{tabular}{|c|c|c|c|c|c|c|}
\hline SNP.Id & Type & Gene.Symbol & Variant.Id & P.Value & Effect & Tissue \\
\hline rs838880 & eQTL & SCARB1 & chr12_124777047_C_T_b38 & $1.5 \mathrm{E}-08$ & -0.20 & Cells - Cultured fibroblasts \\
\hline rs 838880 & sQTL & SCARB1 & chr12_124777047_C_T_b38 & $4.1 \mathrm{E}-06$ & -0.34 & Testis \\
\hline rs737337 & sQTL & DOCK6 & chr19_11236817_T_C_b38 & $3.8 \mathrm{E}-43$ & 0.99 & Artery - Tibial \\
\hline rs737337 & sQTL & DOCK6 & chr19_11236817_T_C_b38 & $6.4 \mathrm{E}-35$ & 0.93 & Adipose - Subcutaneous \\
\hline rs737337 & sQTL & DOCK6 & chr19_11236817_T_C_b38 & $6.4 \mathrm{E}-35$ & 0.93 & Adipose - Subcutaneous \\
\hline rs737337 & sQTL & DOCK6 & chr19_11236817_T_C_b38 & $1.6 \mathrm{E}-27$ & 0.95 & Esophagus - Muscularis \\
\hline rs737337 & sQTL & DOCK6 & chr19_11236817_T_C_b38 & $3.2 \mathrm{E}-20$ & 1.10 & Colon - Sigmoid \\
\hline rs737337 & sQTL & DOCK6 & chr19_11236817_T_C_b38 & $1.1 \mathrm{E}-17$ & 0.93 & Esophagus - Gastroesophageal Junction \\
\hline rs737337 & sQTL & DOCK6 & chr19_11236817_T_C_b38 & $1.8 \mathrm{E}-09$ & 0.81 & Artery - Coronary \\
\hline rs737337 & sQTL & DOCK6 & chr19_11236817_T_C_b38 & $1.2 \mathrm{E}-07$ & -0.49 & Thyroid \\
\hline rs737337 & sQTL & KANK2 & chr19_11236817_T_C_b38 & $4.4 \mathrm{E}-07$ & 0.43 & Artery - Tibial \\
\hline rs737337 & sQTL & KANK2 & chr19_11236817_T_C_b38 & $3.5 \mathrm{E}-06$ & 0.55 & Heart - Left Ventricle \\
\hline rs2943641 & eQTL & IRS1 & chr2_226229029_T_C_b38 & $1.4 \mathrm{E}-16$ & -0.30 & Adipose - Subcutaneous \\
\hline rs2943641 & eQTL & IRS1 & chr2_226229029_T_C_b38 & $6.1 \mathrm{E}-12$ & -0.23 & Adipose - Visceral (Omentum) \\
\hline rs2943641 & eQTL & RP11-395N3.2 & chr2_226229029_T_C_b38 & $3.5 \mathrm{E}-09$ & -0.23 & Adipose - Subcutaneous \\
\hline rs2943641 & eQTL & RP11-395N3.1 & chr2_226229029_T_C_b38 & $2.1 \mathrm{E}-07$ & -0.23 & Adipose - Subcutaneous \\
\hline rs2943641 & eQTL & RP11-395N3.2 & chr2_226229029_T_C_b38 & $2.3 \mathrm{E}-06$ & -0.19 & Adipose - Visceral (Omentum) \\
\hline rs6065904 & eQTL & PLTP & chr20_45906012_G_A_b38 & $4.4 \mathrm{E}-22$ & -0.27 & Muscle - Skeletal \\
\hline rs6065904 & eQTL & PLTP & chr20_45906012_G_A_b38 & $1.6 \mathrm{E}-16$ & -0.27 & Adipose - Subcutaneous \\
\hline rs6065904 & eQTL & PLTP & chr20_45906012_G_A_b38 & $1.2 \mathrm{E}-15$ & -0.28 & Adipose - Visceral (Omentum) \\
\hline rs6065904 & eQTL & PLTP & chr20_45906012_G_A_b38 & $3.2 \mathrm{E}-15$ & -0.42 & Heart - Atrial Appendage \\
\hline rs6065904 & eQTL & PLTP & chr20_45906012_G_A_b38 & $7.2 \mathrm{E}-14$ & -0.25 & Artery - Tibial \\
\hline rs6065904 & eQTL & PLTP & chr20_45906012_G_A_b38 & $1.8 \mathrm{E}-12$ & -0.27 & Nerve - Tibial \\
\hline rs6065904 & eQTL & PLTP & chr20_45906012_G_A_b38 & 7.3E-12 & -0.26 & Esophagus - Muscularis \\
\hline rs6065904 & eQTL & PLTP & chr20_45906012_G_A_b38 & $2.0 \mathrm{E}-11$ & -0.29 & Colon - Transverse \\
\hline rs6065904 & eQTL & PLTP & chr20_45906012_G_A_b38 & $4.1 \mathrm{E}-11$ & -0.32 & Colon - Sigmoid \\
\hline rs6065904 & eQTL & PLTP & chr20_45906012_G_A_b38 & $1.2 \mathrm{E}-09$ & -0.26 & Artery - Aorta \\
\hline rs6065904 & eQTL & PLTP & chr20_45906012_G_A_b38 & $4.2 \mathrm{E}-09$ & -0.29 & Heart - Left Ventricle \\
\hline rs6065904 & eQTL & PLTP & chr20_45906012_G_A_b38 & $5.0 \mathrm{E}-09$ & -0.22 & Thyroid \\
\hline rs6065904 & eQTL & PLTP & chr20_45906012_G_A_b38 & $1.7 \mathrm{E}-08$ & -0.29 & Stomach \\
\hline rs6065904 & eQTL & PLTP & chr20_45906012_G_A_b38 & 4.3E- 08 & -0.24 & Lung \\
\hline rs6065904 & eQTL & NEURL2 & chr20_45906012_G_A_b38 & $6.6 \mathrm{E}-08$ & -0.26 & Adipose - Subcutaneous \\
\hline rs6065904 & eQTL & PLTP & chr20_45906012_G_A_b38 & $6.8 \mathrm{E}-08$ & -0.33 & Liver \\
\hline rs6065904 & eQTL & CTSA & chr20_45906012_G_A_b38 & $4.0 \mathrm{E}-07$ & -0.14 & Nerve - Tibial \\
\hline rs6065904 & eQTL & PLTP & chr20_45906012_G_A_b38 & $5.3 \mathrm{E}-07$ & -0.37 & Spleen \\
\hline rs6065904 & eQTL & NEURL2 & chr20_45906012_G_A_b38 & $5.6 \mathrm{E}-07$ & -0.26 & Adipose - Visceral (Omentum) \\
\hline rs6065904 & eQTL & PLTP & chr20_45906012_G_A_b38 & $8.9 \mathrm{E}-07$ & -0.46 & Small Intestine - Terminal Ileum \\
\hline rs6065904 & eQTL & RP3-337O18.9 & chr20_45906012_G_A_b38 & $1.8 \mathrm{E}-06$ & -0.22 & Adipose - Subcutaneous \\
\hline rs6065904 & eQTL & WFDC3 & chr20_45906012_G_A_b38 & $2.9 \mathrm{E}-06$ & -0.31 & Nerve - Tibial \\
\hline rs6065904 & eQTL & DNTTIP1 & chr20_45906012_G_A_b38 & $3.1 \mathrm{E}-06$ & -0.17 & Artery - Tibial \\
\hline rs6065904 & eQTL & WFDC3 & chr20_45906012_G_A_b38 & $4.5 \mathrm{E}-06$ & -0.27 & Skin - Sun Exposed (Lower leg) \\
\hline rs6065904 & eQTL & SNX21 & chr20_45906012_G_A_b38 & $4.8 \mathrm{E}-06$ & -0.15 & Esophagus - Muscularis \\
\hline rs6065904 & eQTL & WFDC3 & chr20_45906012_G_A_b38 & $8.9 \mathrm{E}-06$ & -0.27 & Skin - Not Sun Exposed (Suprapubic) \\
\hline rs6065904 & eQTL & DNTTIP1 & chr20_45906012_G_A_b38 & $1.0 \mathrm{E}-05$ & -0.14 & Nerve - Tibial \\
\hline
\end{tabular}




\begin{tabular}{|c|c|c|c|c|c|c|}
\hline SNP.Id & Type & Gene.Symbol & Variant.Id & P.Value & Effect & Tissue \\
\hline rs6065904 & eQTL & PLTP & chr20_45906012_G_A_b38 & $1.1 \mathrm{E}-05$ & -0.27 & Prostate \\
\hline rs6065904 & eQTL & PLTP & chr20_45906012_G_A_b38 & $1.3 \mathrm{E}-05$ & -0.26 & Pituitary \\
\hline rs6065904 & eQTL & PLTP & chr20_45906012_G_A_b38 & $1.4 \mathrm{E}-05$ & -0.21 & Esophagus - Gastroesophageal Junction \\
\hline rs6065904 & eQTL & SNX21 & chr20_45906012_G_A_b38 & $1.5 \mathrm{E}-05$ & -0.16 & Esophagus - Mucosa \\
\hline rs6065904 & eQTL & SNX21 & chr20_45906012_G_A_b38 & $1.7 \mathrm{E}-05$ & -0.23 & Colon - Sigmoid \\
\hline rs6065904 & eQTL & SNX21 & chr20_45906012_G_A_b38 & $1.7 \mathrm{E}-05$ & -0.17 & Thyroid \\
\hline rs6065904 & eQTL & PLTP & chr20_45906012_G_A_b38 & $2.6 \mathrm{E}-05$ & -0.21 & Breast - Mammary Tissue \\
\hline rs6065904 & eQTL & WFDC3 & chr20_45906012_G_A_b38 & $2.9 \mathrm{E}-05$ & -0.23 & Artery - Tibial \\
\hline rs6065904 & eQTL & NEURL2 & chr20_45906012_G_A_b38 & $3.2 \mathrm{E}-05$ & -0.21 & Thyroid \\
\hline rs6065904 & eQTL & PLTP & chr20_45906012_G_A_b38 & $3.7 \mathrm{E}-05$ & -0.17 & Testis \\
\hline rs6065904 & eQTL & CTSA & chr20_45906012_G_A_b38 & $4.4 \mathrm{E}-05$ & -0.11 & Skin - Not Sun Exposed (Suprapubic) \\
\hline rs6065904 & eQTL & WFDC3 & chr20_45906012_G_A_b38 & $5.8 \mathrm{E}-05$ & -0.23 & Muscle - Skeletal \\
\hline rs6065904 & eQTL & NEURL2 & chr20_45906012_G_A_b38 & $8.2 \mathrm{E}-05$ & -0.27 & Heart - Atrial Appendage \\
\hline rs6065904 & eQTL & SNX21 & chr20_45906012_G_A_b38 & $8.4 \mathrm{E}-05$ & -0.17 & Artery - Aorta \\
\hline rs6065904 & eQTL & NEURL2 & chr20_45906012_G_A_b38 & $9.5 \mathrm{E}-05$ & -0.24 & Artery - Aorta \\
\hline rs6065904 & eQTL & WFDC3 & chr20_45906012_G_A_b38 & $9.5 \mathrm{E}-05$ & -0.31 & Artery - Aorta \\
\hline rs6065904 & eQTL & RP3-337O18.9 & chr20_45906012_G_A_b38 & $9.5 \mathrm{E}-05$ & -0.29 & Heart - Atrial Appendage \\
\hline rs6065904 & eQTL & PLTP & chr20_45906012_G_A_b38 & $1.2 \mathrm{E}-04$ & -0.15 & Skin - Sun Exposed (Lower leg) \\
\hline rs6065904 & eQTL & WFDC13 & chr20_45906012_G_A_b38 & $1.5 \mathrm{E}-04$ & 0.28 & Esophagus - Muscularis \\
\hline rs6065904 & eQTL & DNTTIP1 & chr20_45906012_G_A_b38 & $2.1 \mathrm{E}-04$ & -0.12 & Cells - Cultured fibroblasts \\
\hline rs6065904 & sQTL & ZNF335 & chr20_45906012_G_A_b38 & $3.3 \mathrm{E}-11$ & -0.65 & Testis \\
\hline rs6065904 & sQTL & ACOT8 & chr20_45906012_G_A_b38 & $1.3 \mathrm{E}-09$ & 0.58 & Heart - Left Ventricle \\
\hline rs6065904 & sQTL & PLTP & chr20_45906012_G_A_b38 & $4.5 \mathrm{E}-08$ & -0.32 & Whole Blood \\
\hline rs6065904 & sQTL & PLTP & chr20_45906012_G_A_b38 & $4.8 \mathrm{E}-08$ & 0.53 & Spleen \\
\hline rs6065904 & sQTL & ACOT8 & chr20_45906012_G_A_b38 & $1.3 \mathrm{E}-07$ & 0.42 & Esophagus - Mucosa \\
\hline rs6065904 & sQTL & ACOT8 & chr20_45906012_G_A_b38 & $2.6 \mathrm{E}-07$ & 0.49 & Heart - Atrial Appendage \\
\hline rs6065904 & sQTL & CTSA & chr20_45906012_G_A_b38 & $1.0 \mathrm{E}-06$ & -0.41 & Artery - Aorta \\
\hline rs6065904 & sQTL & ACOT8 & chr20_45906012_G_A_b38 & $1.2 \mathrm{E}-06$ & 0.33 & Nerve - Tibial \\
\hline rs6065904 & sQTL & ACOT8 & chr20_45906012_G_A_b38 & $1.2 \mathrm{E}-06$ & 0.67 & Brain - Spinal cord (cervical c-1) \\
\hline rs6065904 & sQTL & TNNC2 & chr20_45906012_G_A_b38 & $2.1 \mathrm{E}-06$ & 0.54 & Brain - Cerebellum \\
\hline rs6065904 & sQTL & ACOT8 & chr20_45906012_G_A_b38 & $2.1 \mathrm{E}-06$ & 0.54 & Brain - Cerebellum \\
\hline rs6065904 & sQTL & WFDC3 & chr20_45906012_G_A_b38 & $5.5 \mathrm{E}-06$ & 0.23 & Skin - Sun Exposed (Lower leg) \\
\hline rs6065904 & sQTL & WFDC3 & chr20_45906012_G_A_b38 & $9.4 \mathrm{E}-06$ & -0.28 & Skin - Not Sun Exposed (Suprapubic) \\
\hline
\end{tabular}

